\title{
High Fidelity and Efficient Computations of Dynamic Loads for Multidisciplinary Design Optimization of Flexible Transport Aircraft
}

\author{
by \\ Michelle Guzman Nieto \\ BSc in Aeronautical Engineering
}

A thesis submitted to the Faculty of Graduate and Post Doctoral Affairs in partial fulfillment of the requirements for the degree of Master of Applied Science in

Aerospace Engineering.

Carleton University

Ottawa, Ontario

(C) 2018

Michelle Guzman Nieto 
The current state-of-the-art design optimization of airframes is tightly wounded to its loads analyses as the process is usually conducted employing a deterministic set of critical load cases. The sheer number of scenarios required to estimate the critical loading conditions prevent these two processes from integrating, obstructing the development of a Multidisciplinary Design Optimization (MDO) framework. In this thesis, the problem of high fidelity and efficient estimation of critical dynamic aeroelastic loads is addressed, as a first step, towards the development of an integrated MDO platform for airframes at preliminary and detailed design stages. The method is based on the Kriging metamodeling technique along with the Latin Hypercube scheme for initial sampling and the expected improvement function for subsequent selection of sample points, known formally as the Efficient Global Optimization (EGO) algorithm. Furthermore, different inexpensive metrics, based on the concept of modal contribution factors, are investigated to serve as indicators to determine if a substantial change in the loads has occurred during the design optimization cycle, triggering the requirement for the re-exploration of the loads design space. A case study is presented to evaluate the performance of the proposed methodology versus a full factorial search. A reduction of $84 \%$ was achieved in the total time of execution employing the proposed methodology.

Keywords: Aeroelasticity; Design Optimization; Kriging Metamodeling Predictor, Efficient Global Optimization; Multidisciplinary Optimization; Aircraft loads; Modal Participation Factors. 
This research project is an original work developed by Michelle Guzman Nieto, under the supervision of the Professor Mostafa El Sayed, conforming to all mandatory requirements as stated by Carleton University.

The project is part of the Bombardier Aerospace initiative to develop a multi disciplinary framework for optimization of aircraft wing-box. In close collaboration, Bombardier Aerospace provided data relevant to the aeroelastic numerical model, which is used as part of the case study in chapter 6. All numerical data provided has been normalized to protect the integrity of our partner's' design.

Two conference papers: Guzman, M., ElSayed, M.S.A., Walch, D. (2018), “Improved modal contribution factors as response tracking mechanisms for dynamic systems during design optimization" and Guzman, M., ElSayed, M.S.A., Walch, D. (2018), "Modal participation factors and their potential applications in aerospace: a review" presented during the CSME 2018 international conference at York University, Toronto, Ontario, Canada, were partially reproduced here under Chapter 4. 


\section{ACKNOWLEDGMENTS}

This research is the result of the effort, the dedication and, above all, the commitment that scientific work demands. It is a pleasure to thank those who made this research possible; firstly, to my supervisor Mostafa El Sayed, whose guidance and support were crucial for the development of this project.

To Denis Walch and the engineers in the loads group at Bombardier Aerospace, whose brief encounter enabled me to shape the final product of this research. To Carleton's administrative personal for their constant assistance and to the professors I had the opportunity to learn from. And finally, to MITACS, CARIC and Bombardier Aerospace for their financial support.

I also want to thank my family and friends, for their affection and constant encouragement. Specially, to my partner, David Kohoko, for his love and unconditional support. And finally, to my mother, Myriam Nieto for teaching me that discipline, courage and will are the driving force of dreams. 


\section{TABLE OF CONTENTS}

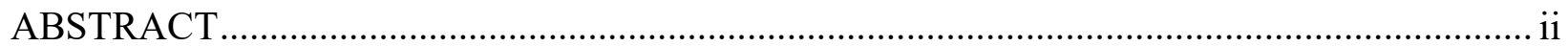

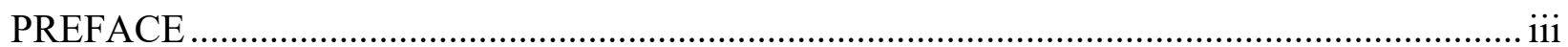

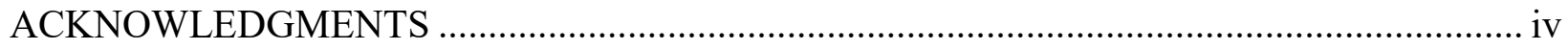

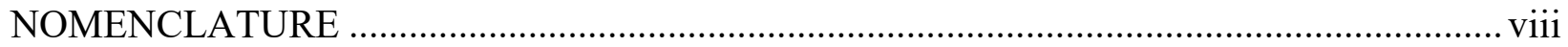

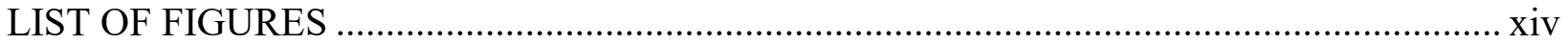

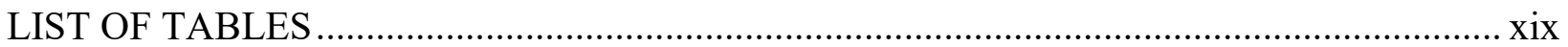

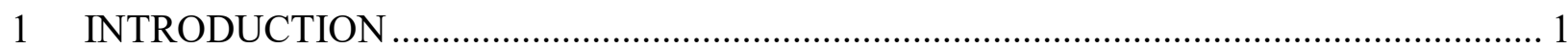

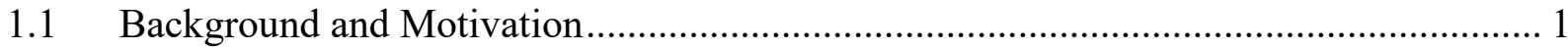

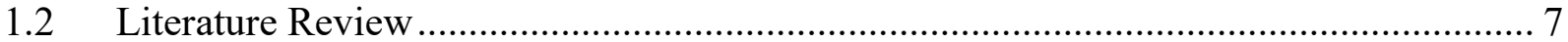

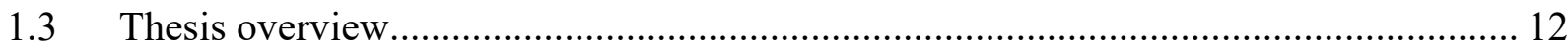

2 THEORETICAL FRAMEWORK PART I: NUMERICAL MODEL FOR FLEXIBLE

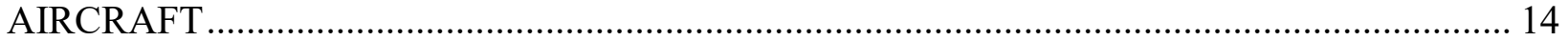

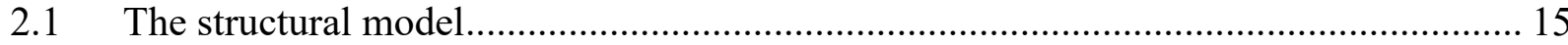

2.1.1 Global Finite Element Model........................................................................... 16

2.1.2 Model Order Reduction - The Stick Model …................................................ 16

2.1.3 Modal analysis and the generalized coordinates............................................. 19

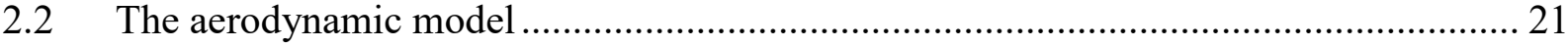

2.2.1 The Doublet-Lattice panel method ............................................................. 21 
2.3 Splining interpolation - Transfer of aerodynamic forces to the structural grid 24

2.4 The aeroelastic equation in modal coordinates ................................................ 25

2.5 Atmospheric disturbances for commercial transport aircraft .................................... 28

2.5.1 Gust and Turbulence models ...................................................................... 29

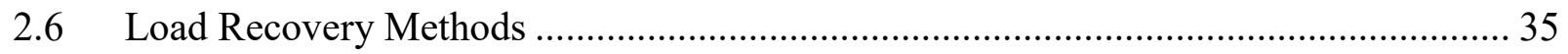

2.6.1 Mode displacement method .................................................................... 35

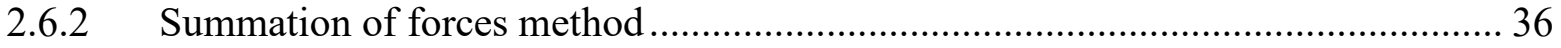

2.6.3 Selection of critical loads and the loads envelope ............................................ 36

3 THEORETICAL FRAMEWORK PART II: EFFICIENT GLOBAL OPTIMIZATION (EGO) 39

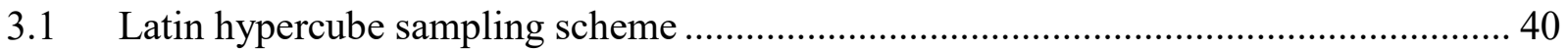

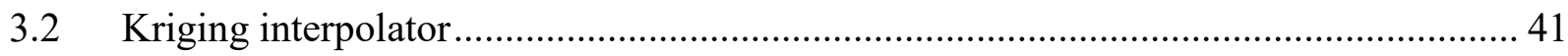

3.2.1 The Gaussian Correlation Model ................................................................... 45

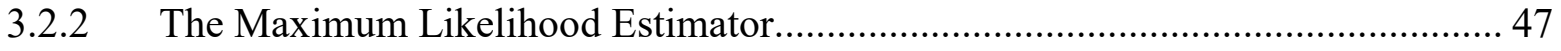

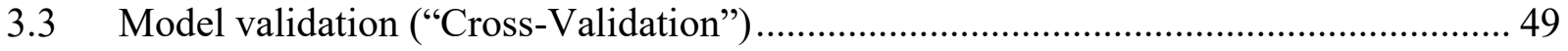

3.4 The Expected Improvement Function ............................................................... 50

4 THEORETICAL FRAMEWORK PART III: MODAL CONTRIBUTION FACTORS...... 52

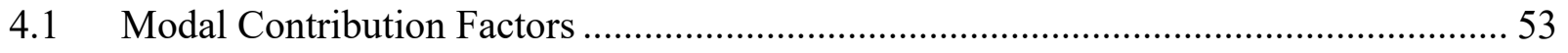

4.1.1 Class I: Static Modal Participation Factors (SMPF) ...................................... 54

4.1.2 Class II: Relative Importance of a Mode ................................................... 61 


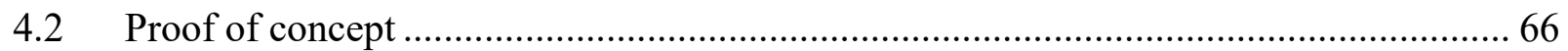

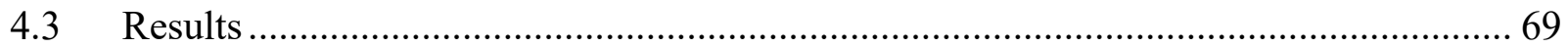

4.4 Best Choice of metric for the surrogate-aided response algorithm ............................ 73

5 IMPLEMENTATION OF THE SURROGATE-AIDED RESPONSE ALGORITHM .......... 75

5.1 Element Modal Strain Energy correlation function application................................ 77

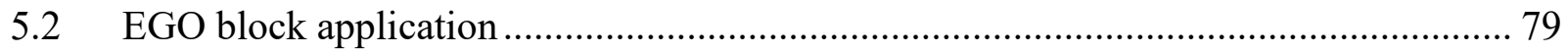

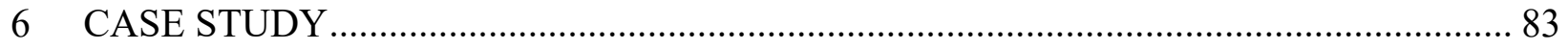

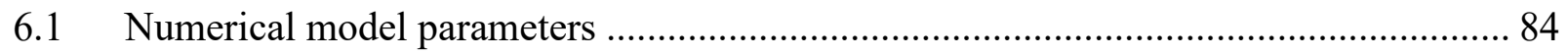

6.1.1 Parametrized mass and stiffness variations.................................................. 85

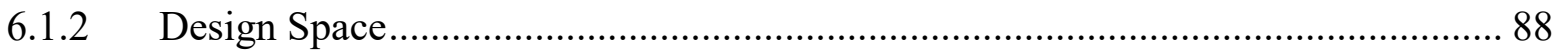

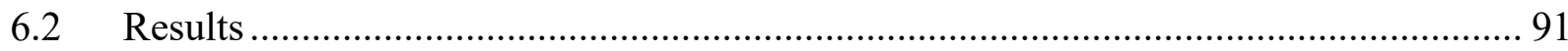

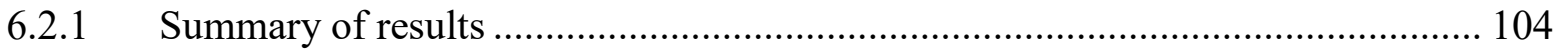

7 CONCLUSIONS AND FURTHER WORK ........................................................... 106

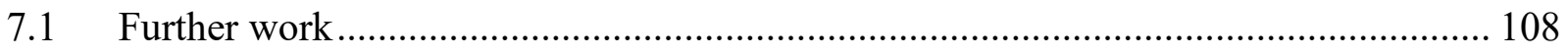

APPENDIX I: SURROGATE-AIDED LOADS ALGORITHM ....................................... 109

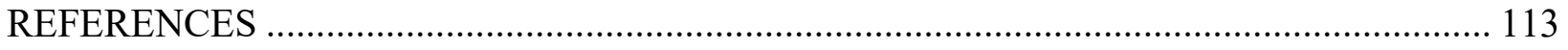


Capital letters

A

$\left[A_{j j}\right]$

$C_{L_{\alpha}}$

$\left[C_{q}\right]$

$\operatorname{Cov}[Z(X)]$

$\left[D_{j k}^{1}\right]$

$\left[D_{j k}^{2}\right]$

E

$E_{I}$

$E[I(\boldsymbol{X})]$

F

$F_{g}$

G

$\left[G_{k g}\right]$

$I(X)$

$I_{z}, I_{y}$

\section{Definition}

Equivalent cross section area

Matrix of aerodynamic influence coefficients on lifting

elements

Lift coefficient at angle of attack $\alpha$

Modal damping matrix

Covariance of the stationary random error

Real part of substantial differentiation matrix;

dimensionless

Imaginary part of substantial differentiation matrix;

dimensionless

Modulus of elasticity

Expected improvement threshold

Expected improvement function

Matrix of polynomial functions $f(\boldsymbol{X})$ evaluated at the sample points

Flight profile alleviation factor

Modulus of rigidity

Spline matrix

Improvement function

Second moment of area about the $y$ and $\mathrm{z}$ axes 


\begin{tabular}{cl}
$J$ & Second polar moment of area \\
$K_{g}$ & Old gust alleviation factor \\
{$[K]$} & Stiffness matrix \\
{$\left[K_{e}\right]$} & Element stiffness matrix in the global coordinate system \\
{$\left[K_{q}\right]$} & Modal stiffness matrix \\
$L$ & Lift force \\
$L\left[\theta, \beta, \sigma^{2} \mid Y\right]$ & Maximum likelihood estimator \\
{$[M]$} & Mass matrix \\
{$[M]_{q}$} & Modal mass matrix \\
$M S E_{j i}$ & Element modal strain energy participation factor \\
$M S E C_{j}$ & Element modal strain energy coefficient \\
$\boldsymbol{P}$ & $n \times p$ matrix of random permutations \\
$\{P(t)\}$ & Forcing function in the time domain \\
$\{P(\omega)\}$ & Forcing function in the frequency domain \\
{$\left[S_{k j}\right]$} & Matrix of Force Aerodynamic Influence coefficients on the \\
{$\left[Q_{k k}\right]$} & aerodynamic grid points \\
{$\left[Q_{q}\right]$} & Matrix of Force Aerodynamic Influence coefficients in the \\
$\boldsymbol{R}\left(\boldsymbol{\theta}, \boldsymbol{x}_{\boldsymbol{i}}, \boldsymbol{x}_{\boldsymbol{j}}\right)$ & modal coordinates \\
$S$ & $n \times p$ matrix of randomly distributed numbers $[0,1]$. \\
& Wing planform area \\
\hline &
\end{tabular}




\begin{tabular}{cl}
\multicolumn{1}{c}{$S^{*}$} & Wing semi-span \\
$S C V R$ & Standardized cross-validated residual \\
$V_{\infty}$ & Free stream velocity \\
$W$ & Airplane weight \\
$\boldsymbol{X}$ & Set of known sample points \\
$\boldsymbol{Y}_{\boldsymbol{t}}(\boldsymbol{X})$ & True function \\
$\boldsymbol{Y}(\boldsymbol{X})$ & Set of responses of the sample points \\
$\widehat{\boldsymbol{Y}}(\boldsymbol{X})$ & Kriging predictor \\
$\boldsymbol{Z}(\boldsymbol{X})$ & Gaussian stationary random error
\end{tabular}

\section{Minuscule letters Definitions}

$\begin{array}{cl}b & \text { Reference semi-chord } \\ e & \text { Error between the true and the estimated res } \\ f(\boldsymbol{X}) & \text { Set of polynomial regression functions } \\ \left\{f_{j}\right\} & \text { Pressure on each lifting element (box) } \\ d_{x_{i}}, d_{y_{i}}, d_{z_{i}} & \begin{array}{l}\text { Displacements due to a unitary force in the } \mathrm{x} \\ \text { global axes. } \\ h\end{array} \\ h_{m o} & \text { Height of the Stick's model beam elements } \\ k & \text { Maximum operating altitude } \\ k_{q_{j}} & \text { Reduced frequency } \\ \left(k_{z}\right)_{i-j},\left(k_{x}\right)_{i-j} & \text { Shear factors per bay station } \\ l & \text { Scale of the turbulence }\end{array}$




\begin{tabular}{|c|c|}
\hline$m_{q_{j}}$ & Modal mass of the $j t h$ mode \\
\hline$n$ & Number of sample points \\
\hline$p$ & Number of dimensions of the design space \\
\hline$q(t)$ & Generalized or Modal coordinates \\
\hline $\bar{q}$ & dynamic pressure \\
\hline$r$ & Distance penetrated into the gust \\
\hline$r(\boldsymbol{X})$ & Correlation vector between unknown and known points. \\
\hline$\{u(t)\}$ & Vector of physical displacements \\
\hline$u_{g}$ & $\begin{array}{l}\text { Displacements at the reduced order model structural grid } \\
\text { points }\end{array}$ \\
\hline$u_{k}$ & Displacements at the aerodynamic grid points \\
\hline$w$ & Width of the Sticks model beam elements \\
\hline$w_{g}$ & Gust Normal velocity \\
\hline$w_{j}$ & $\begin{array}{l}\text { Normal flow (downwash) on each lifting element; } \\
\text { dimensionless }\end{array}$ \\
\hline$w_{o}$ & Gust equivalent air speed \\
\hline$w_{\text {ref }}$ & Gust reference air speed \\
\hline$w_{j}^{g}$ & $\begin{array}{l}\text { Static aerodynamic downwash on each lifting element; } \\
\text { dimensionless }\end{array}$ \\
\hline$y_{j}^{*}$ & $\begin{array}{l}y \text { coordinate at the } j t h \text { station in the wing local coordinate } \\
\text { system }\end{array}$ \\
\hline$x, y, z$ & Wing local coordinate system \\
\hline$z(\boldsymbol{X})$ & $\begin{array}{l}\text { Gaussian stationary random process evaluated at the } \\
\text { unknown points. }\end{array}$ \\
\hline
\end{tabular}




\begin{tabular}{|c|c|}
\hline Capital Greek letters & Definition \\
\hline$\Phi$ & Matrix of eigenvectors \\
\hline$\Psi(\cdot)$ & Standard normal distribution function \\
\hline$\Omega$ & Reduced frequency \\
\hline Minuscule Greek letters & Definition \\
\hline$\beta$ & $\begin{array}{l}\text { Unknown constant coefficients solved through least squares } \\
\text { method. }\end{array}$ \\
\hline$\gamma$ & Monitored load threshold \\
\hline$\zeta(X)$ & $\begin{array}{l}\text { Unknown constant coefficients of the simple linear } \\
\text { predictor. }\end{array}$ \\
\hline$\Delta \eta$ & Old incremental load factor \\
\hline$\eta_{j}$ & $\begin{array}{l}\text { Location of each wing station } \\
y_{j}^{*} \text { normalized with respect to the semi-span } S^{*}\end{array}$ \\
\hline$\theta_{x}, \theta_{y}, \theta_{z}$ & Deformation rotational angle around $\mathrm{x}, \mathrm{y}$, and $\mathrm{z}$ \\
\hline$\theta$ & Undefined parameter of the spatial correlation function \\
\hline$\mu_{g}$ & Mass ratio \\
\hline$\lambda$ & Lagrange multipliers \\
\hline$\lambda_{g}$ & Gust wave length \\
\hline$\rho$ & Air density \\
\hline$\sigma_{w}$ & Gust RMS velocity \\
\hline$\sigma^{2}$ & Variance of the Gaussian stationary random process \\
\hline$\left\{\varphi_{i}\right\}$ & Mode shape vector of the $i$-th mode \\
\hline$\phi(X)$ & Mean square error \\
\hline
\end{tabular}


$\psi(\cdot)$

$\omega$

$\omega_{n_{i}}$
Standard normal density

Angular or circular frequency

Natural angular frequency of the $i$-th mode 


\section{LIST OF FIGURES}

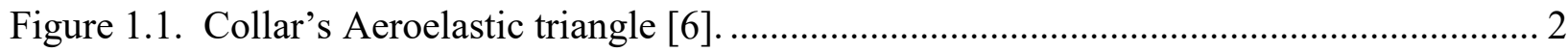

Figure 1.2. Schematic of the current industrial process the loads analysis and the structural

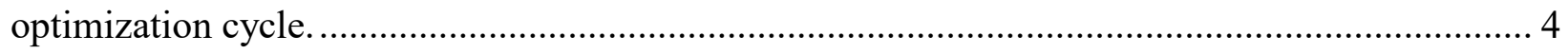

Figure 1.3 Simplified block diagram of the current industrial process...................................... 4

Figure 1.4. Schematic of the proposed process: a multidisciplinary design optimization process. 6

Figure 1.5. Simplified block diagram of the proposed process. The block framed with a dashed line indicates the focus of this research: The Surrogate-aided loads analysis................................. 6

Figure 2.1. Visual representation of a GFEM (on the left) transformed to a low-fidelity model (on

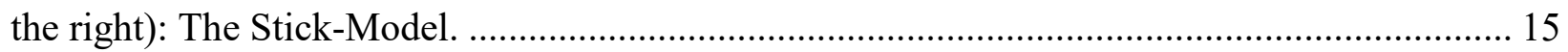

Figure 2.2. Wing box axes used to extract the stiffness properties........................................... 17

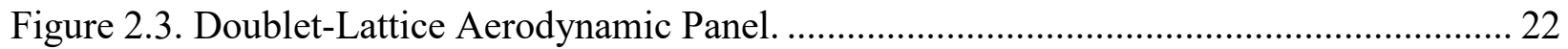

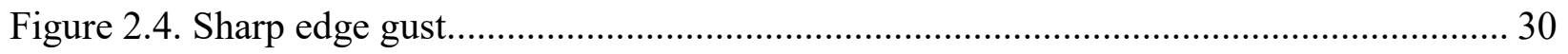

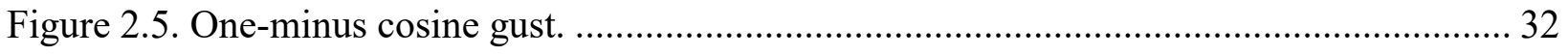

Figure 2.6. A) Time history of the twist moment. B) 1-D load envelope: twist moment along the wing

Figure 2.7. Convex hull of correlated loads of a commercial transport aircraft at the wing root. 38

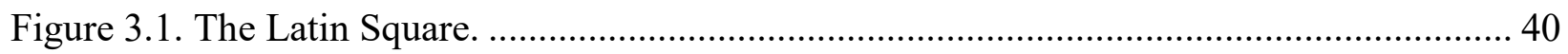

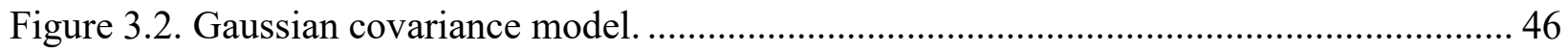

Figure 3.3. Standardized Cross-Validated Residual. One sample point is removed from the sample vector and the surface response is re-interpolated using the Kriging. ........................................ 49 
Figure 4.1. Time history of a dynamic system. The black dots indicate the points computed by the steady-state response participation factor. 57

Figure 4.2. Time history of a dynamic system. The black dots indicate the points computed by the transient response I participation factor. 58

Figure 4.3. Time history of a dynamic system. The black dots indicate the points computed by the transient response II participation factor. 60

Figure 4.4. Proof of concept: the cantilever beam model. 67

Figure 4.5. Solution of the modal equations of motion. A) Presents the behavior of the generalized coordinates. B) Presents the physical displacement of the grid points numbered according to Figure 4.4 68

Figure 4.6. Time history of the element in-plane shear force due to a 1 minus cosine shock of 0.28 seconds. 68

Figure 4.7. Comparison between five different definitions of Modal Participation Fractions..... 69 Figure 4.8. Normalized element in-plane shear force modal participation fraction: A) element 1; B) element 2; C) element 3; D) element 4; E) element 5. 71

Figure 5.1. Overview of the Algorithm. 77

Figure 5.2. Block diagram of the dummy optimization, required to construct the correlation model between the MSEC, as per equation 4.37, and a response of interest. 78

Figure 5.3. EGO Implementation: Estimated maximum out of plane bending moment (normalized) as a function of gust wavelength and half-wing length. The scatter points are the observations (207 points out of 627) used to reconstruct the surface response. 80 Figure 5.4. QQ plot of the standard error for the estimated maximum out of plane bending moment (normalized) as a function of gust wavelength and half-wing length 80 
Figure 5.5. True percentile error between the real and the estimated maximum out of plane bending moment (normalized) as a function of gust wavelength and half-wing length 81

Figure 5.6. EGO Implementation: Estimated multimodal test function. The scatter points are the observations (664 points out of 1681) used to reconstruct the surface response. 81

Figure 5.7. QQ plot of the standard error for the estimated multimodal test function. 82

Figure 5.8. True percentile error between the real and the estimated multimodal test function. . 82

Figure 6.1. Aeroelastic model of a Bombardier Aerospace Platform. 84

Figure 6.2. Percentile change in mass versus percentile change in axial stiffness (EA) as a result of skin thickness reduction in the wing box. Data retrieved with permission of Bombardier Aerospace [104]. 85

Figure 6.3. Percentile change in mass versus percentile change in shear stiffness about the y axis (EIy) as a result of skin thickness reduction in the wing box. Data retrieved with permission of Bombardier Aerospace [104]...... 86

Figure 6.4. Percentile change in mass versus percentile change in shear stiffness about the $\mathrm{z}$ axis (EIz) as a result of skin thickness reduction in the wing box. Data retrieved with permission of Bombardier Aerospace [104] 86

Figure 6.5. Percentile change in mass versus percentile change in torsional stiffness (GJ) as a result of skin thickness reduction in the wing box. Data retrieved with permission of Bombardier

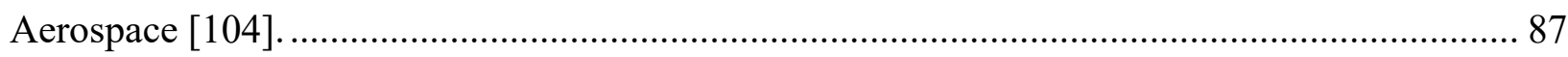

Figure 6.6. Family of gust wavelengths according to FAR25.341 regulations. ........................... 89

Figure 6.7. Gust reference velocity according to FAR25.341 regulations. ................................. 90

Figure 6.8. Flight Profile Alleviation Factor per FAR25.341 regulations................................... 90 
Figure 6.9. Normalized loads versus MSEC at the wing root, $y=0$. The scatter points are the correlated data and the solid lines are the polynomial fits for the following loads: A) Maximum out of plane bending moment (MOPB); B) Maximum out of plane shear force (MOPS); C) Maximum torsion moment (MT); D) Minimum out of plane bending moment (mOPB); E) Minimum out of plane shear force (mOPS); F) Minimum torsion moment (mT). 91

Figure 6.10. Normalized loads versus MSEC at $y=0.25$. The scatter points are the correlated data and the solid lines are the polynomial fits for the following loads: A) Maximum out of plane bending moment (MOPB); B) Maximum out of plane shear force (MOPS); C) Maximum torsion moment (MT); D) Minimum out of plane bending moment (mOPB); E) Minimum out of plane shear force (mOPS); F) Minimum torsion moment (mT). 92 Figure 6.11. Normalized loads versus MSEC at $y=0.50$. The scatter points are the correlated data and the solid lines are the polynomial fits for the following loads: A) Maximum out of plane bending moment (MOPB); B) Maximum out of plane shear force (MOPS); C) Maximum torsion moment (MT); D) Minimum out of plane bending moment (mOPB); E) Minimum out of plane shear force (mOPS); F) Minimum torsion moment (mT) ………….......................................... 92 Figure 6.12. Normalized loads versus MSEC at $y=0.75$. The scatter points are the correlated data and the solid lines are the polynomial fits for the following loads: A) Maximum out of plane bending moment (MOPB); B) Maximum out of plane shear force (MOPS); C) Maximum torsion moment (MT); D) Minimum out of plane bending moment (mOPB); E) Minimum out of plane shear force (mOPS); F) Minimum torsion moment (mT). 93

Figure 6.13. Normalized loads versus MSEC at the wing tip, $y=1.0$. The scatter points are the correlated data and the solid lines are the polynomial fits for the following loads: A) Maximum out of plane bending moment (MOPB); B) Maximum out of plane shear force (MOPS); C) 
Maximum torsion moment (MT); D) Minimum out of plane bending moment (mOPB); E) Minimum out of plane shear force (mOPS); F) Minimum torsion moment (mT) ....................... 93

Figure 6.14. Monitored elements and its normalized location with respect to the wing root location.

Figure 6.15. Normalized estimated max out of plane bending moment at the wing root $(y=0.00)$ during the first iteration as a function of Mach number and altitude with an Expected Improvement threshold of 1 , with 97 sample points 98

Figure 6.16. QQ plot of the max out of plane bending moment at the wing root with 84 initial sample points selected using the LH scheme. 98

Figure 6.17. Percentile error of the interpolated max out of plane bending moment at the wing root as a function of Mach number and altitude with an Expected Improvement threshold of 1. 99 Figure 6.18. Variation of the normalized out of plane bending moment of the time consistent loads as a function of normalized half-wing length. 103

Figure 6.19. Maximum out of plane bending moment (MOPB) at the wing root $(y=0.0)$ as a function of the iteration number. 103

Figure 6.20. Minimum out of plane bending moment (mOPB) at the wing root $(y=0.0)$ as a function of the iteration number. 104 


\section{LIST OF TABLES}

Table 4.1. Estimation of the peak load for an undamped 1-cosine pulse forcing function. 70

Table 6.1. Design Space 88

Table 6.2. Combination of element and load type that triggered the re-exploration of the design space per iteration. 95

Table 6.3. Normalized response surfaces of the 6 monitored loads for the first and the last iteration evaluated at the wing root $(y=0.0)$ and the wing tip $(y=1.0)$.

Table 6.4. Comparison of the performance between the full factorial search and the proposed methodology. The * indicates that $\mathrm{n}$ simulations where run whenever the MSEC trigger was surpassed. 104

Table 6.5. Mean percentage error of the interpolated surfaces during the first iteration. 105 


\section{INTRODUCTION}

Aircraft design is driven by multiple functional requirements that define its mission profile, including endurance, maximum payload, fuel efficiency and airworthiness, among others. During the conceptual design phase, a rough estimate of the aircraft loads is required for the selection of the airframe structural layout. Later, during the preliminary and the detailed design phases, accurate predictions of aircraft design and fatigue loads are needed for structural optimization and certification purposes [1-3].

In the following subsections, a brief discussion about the motivation for the development of the current thesis is provided, followed by a historical review of some of the most important events that set the base for the current status quo on the loads analysis for commercial transport aircraft, along with the latest efforts to integrate the loads analysis into the structural optimization cycle. An overview of this thesis is provided as well, to help navigate the reader through this document.

\subsection{BACKGROUND AND Motivation}

The uncertainty of the loading conditions that the aircraft will experience during flight and the detailed representation of numerical models, makes the process of identifying the design loads (also referred to as critical loads), both time and computationally expensive, since thousands of scenarios need to be analyzed to ensure the limit loads are properly identified $[4,5]$.

In this context, loads are the set of internal forces (i.e., shear force, bending and torsional moments) the airframe experiences as a response to an external excitation. For example, during ground maneuvers, such as taxiing, the interaction between inertial and elastic forces produce a net set of 
internal forces. The focus of this thesis is mainly on dynamic aeroelastic response analysis in which inertial, aerodynamic, and elastic forces interact. Though gust is the most widely used numerical example of aerodynamic turbulence in dynamic aeroelasticity, other incidences also fall under the same category, such as wind-milling analysis. This information is summarized for the reader in the Collar's aeroelastic triangle [6], shown in Figure 1.1.

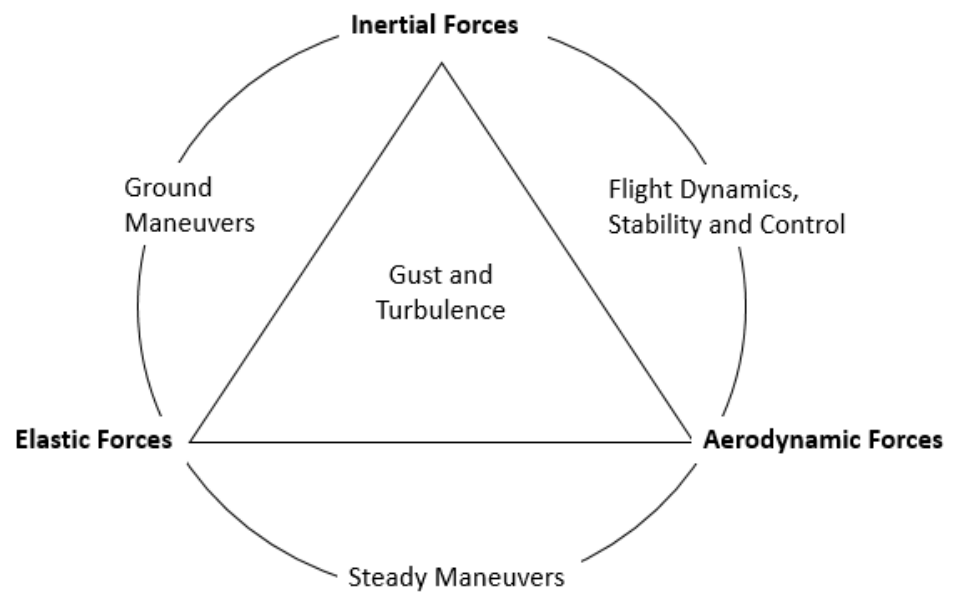

Figure 1.1. Collar's Aeroelastic triangle [6].

The dynamic aeroelastic response is evaluated from the aircraft's equilibrium position, such that the total loads are obtained by adding the steady maneuver load and the incidental response at each given center of gravity position, payload, altitude, and Mach number [6-8], where each combination of parameters is a discrete operational scenario and comprises the design space.

The current state-of-the-art structural optimization is performed on the Detailed Finite Element Model (DFEM). During the loads analysis, as shown in Figure 1.2, the DFEM is replaced by a low fidelity model, known as the Stick Model (also referred to as Reduced Order Model (ROM)), which preserves the dynamic properties of the structure within a frequency range of interest. A 
linearized potential flow method is superimposed to model the aerodynamics and the coupling between the two models is performed through splining techniques. Then, the equations of motion are solved and the corresponding internal loads are recovered at each station (element) of the ROM. The analyzed load cases are then monitored and the critical loading conditions at each station are identified. From this set, a sub-set of time consistent loads is computed and transformed into discretized loads, which are used for sizing and/or design optimization of the airframe.

A simplified block diagram of this process is presented in Figure 1.3. Here, the optimization process is separate from the loads analysis. As a consequence, the set of critical loads of the optimized model are different than the previous set, since several structural components are modified in size and geometry (sometimes even material) altering their static and dynamic aeroelastic behaviour. And therefore, the magnitude of their dynamic response and corresponding internal loads. If the new set of loads have a higher magnitude than those of the previous iteration, both, the design, and the certification requirements are compromised.

Though the use of reduced order models alleviates the computational burden, the integration of the loads analysis into the optimization process is still prohibitively expensive due to the extremely large number of operational scenarios required to be investigated. 


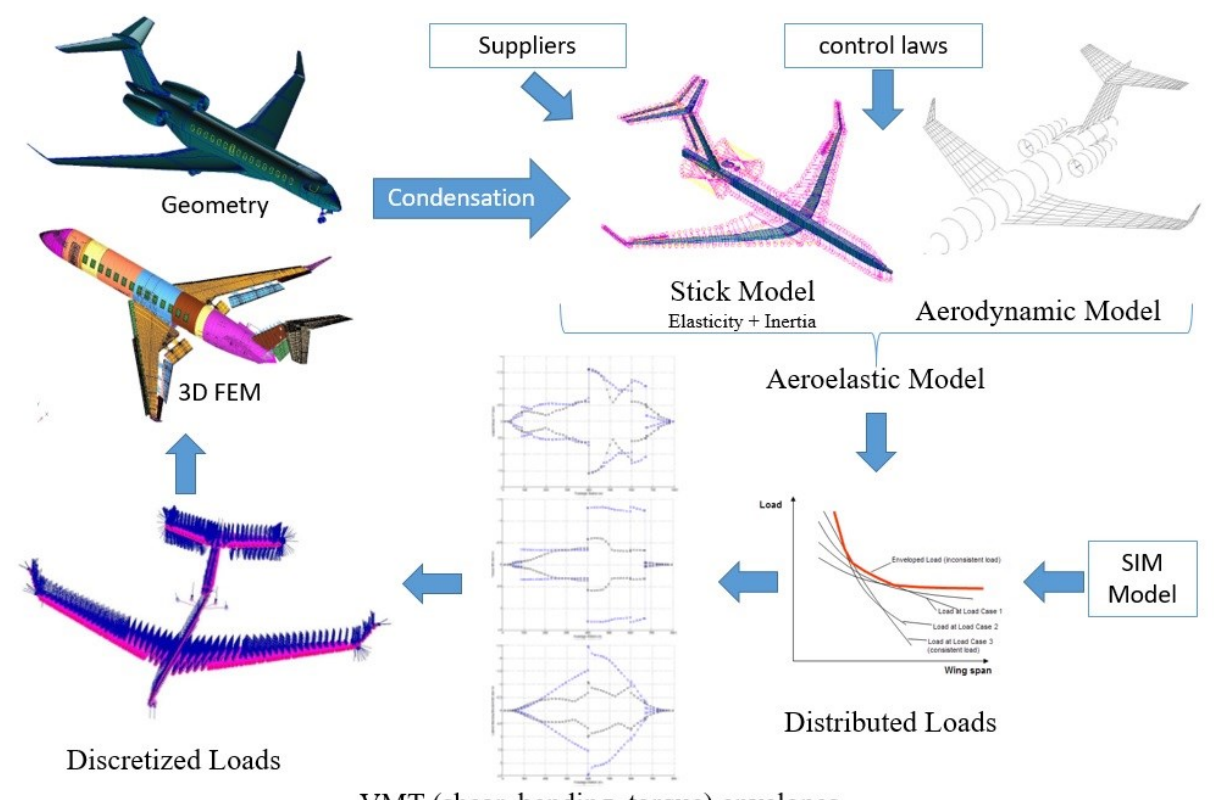

VMT (shear, bending, torque) envelopes

Figure 1.2. Schematic of the current industrial process the loads analysis and the structural optimization cycle.

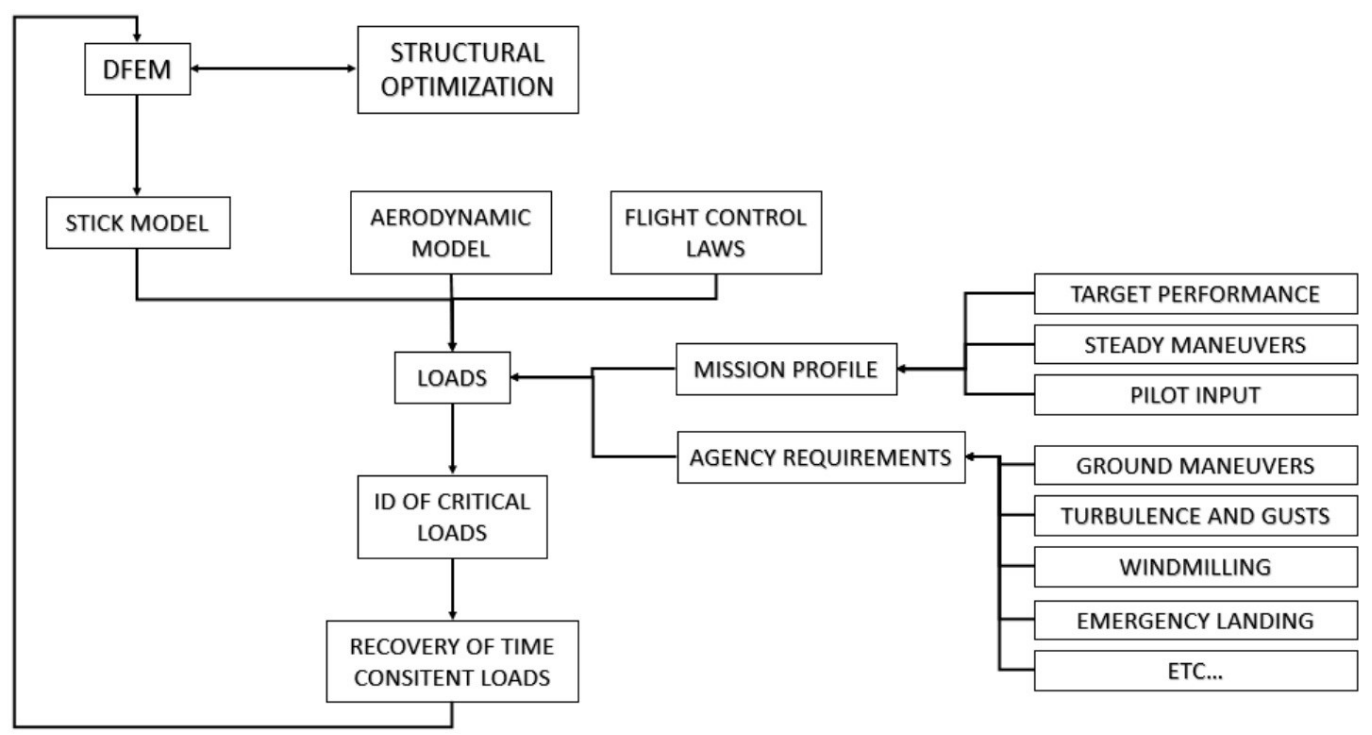

Figure 1.3 Simplified block diagram of the current industrial process. 
In fact, several authors have addressed the need to center, at the core of airplane design, the accurate and efficient computation of loads. Firstly, with the purpose of finding an optimal design with minimum weight, and secondly, to create a leaner industrial process $[1,3,9]$. With new environmental regulations on their way, new aircraft configurations must be explored. Thus, the efficient and high-fidelity prediction of aircraft loads becomes paramount in fulfilling this objective.

In this thesis, the focus lies in the efficient and accurate computation of the aeroelastic loads. This is one of the many challenges that lie in the path of developing an integrated MDO platform, such as the one shown in Figure 1.4. There, the optimization cycle is no longer separated from the loads analysis. Instead as the structural properties vary, the new set of critical or design loads are computed and reapplied to the DFEM. Figure 1.5 shows the simplified block diagram of the integrated process. The highlighted blocks represent the proposed modifications and the dashed blocks represent the obsolete processes.

Through out his work, the mathematical foundation and the practical application of the block named Surrogate-Aided Response, highlighted in green in Figure 1.5, are discussed. This algorithm was design to answer two fundamental questions: how can such a large design space be explored in a more efficient way and how significant is the change in the loading conditions after structural modification. The Efficient Global Optimization (EGO) algorithm [10] is used to answer the first question, whereas the concept of Modal Contribution Factors (MCF) is explored to address the second question. 


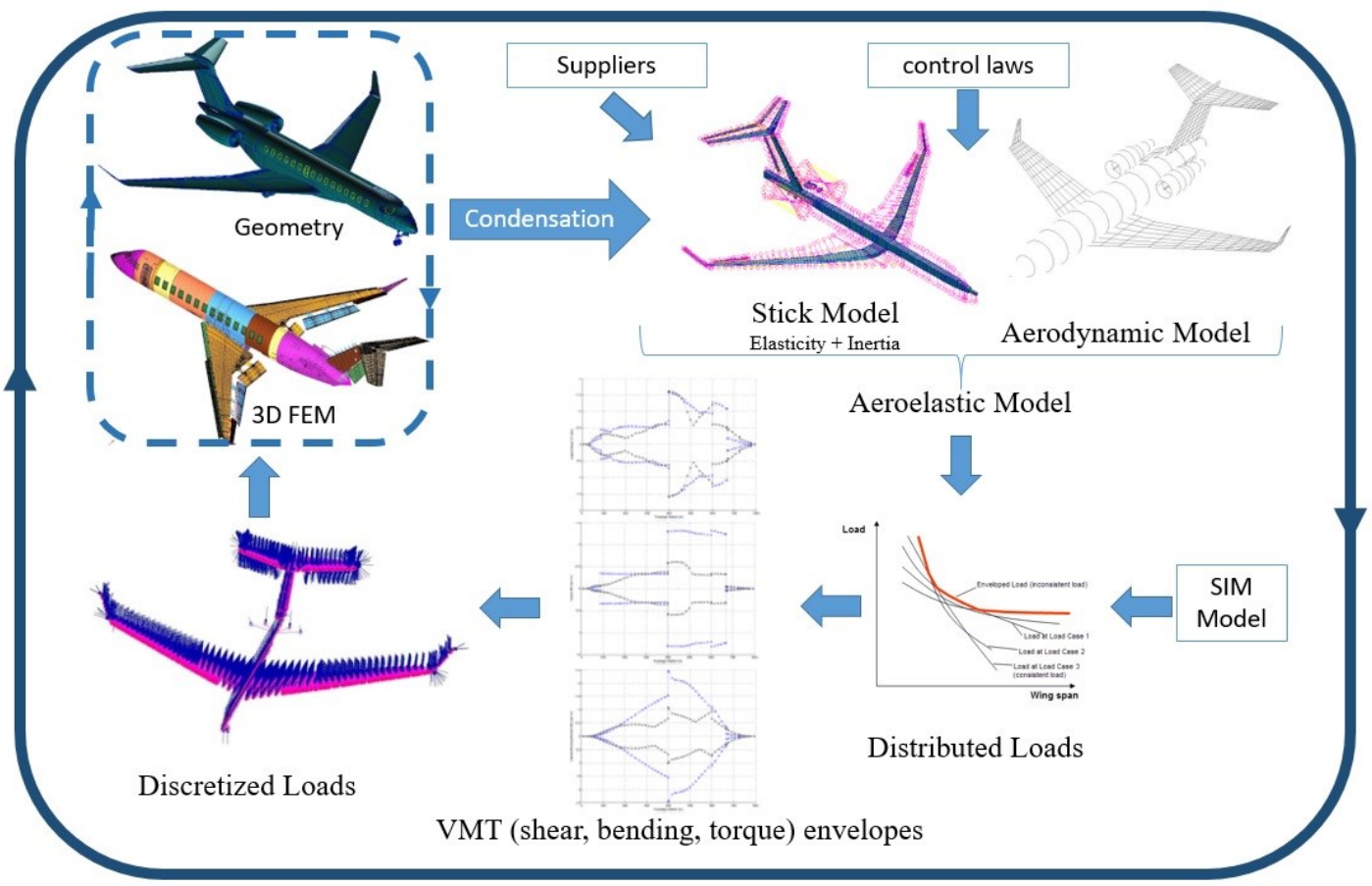

Figure 1.4. Schematic of the proposed process: a multidisciplinary design optimization process.

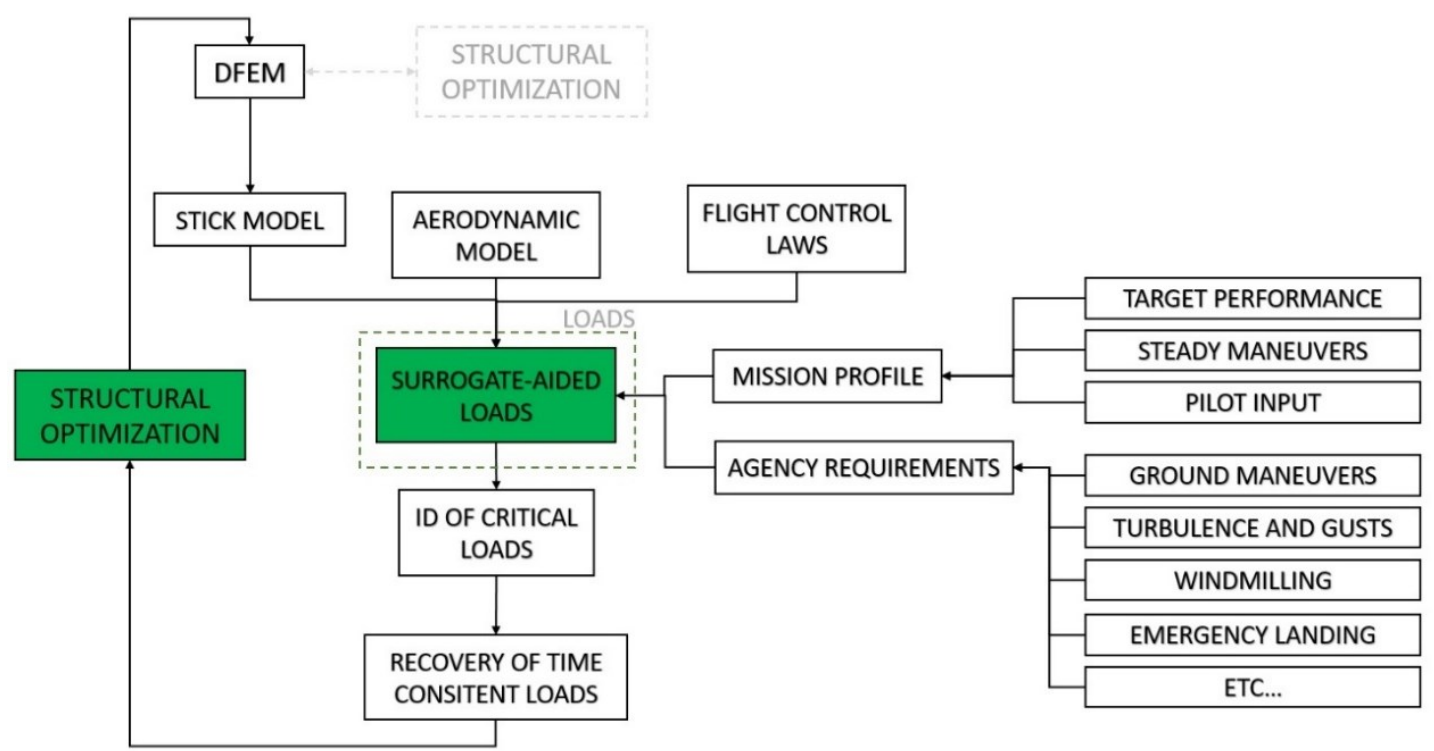

Figure 1.5. Simplified block diagram of the proposed process. The block framed with a dashed line indicates the focus of this research: The Surrogate-aided loads analysis. 


\subsection{LiteratURE REVIEW}

The development of the aircraft critical loads analysis is widely bounded by the development of the airworthiness requirements, as well as by aircraft manufacturers' internal requirements. It was in 1921 when the National Advisory Committee for Aeronautics (NACA) (which later would be known as NASA) first published a technical note requiring 4 critical load cases to be analyzed for army aircraft [11]. As new design configurations appeared, and cruise velocities increased during the early 1930's, the first U.S. airworthiness requirements considering a discrete sharp-edge gust analysis were published [3], though the first theoretical studies of airplanes encountering gust had been published by NACA in 1915 [12].

In 1956, the regulations required an estimate of a static load factor which accounted for the dynamic response of the aircraft $[3,12-13]$ based on a discrete one-minus-cosine gust analysis that was intended to represent more closely the gust velocity gradients in estimating the limit loads. It was during this period when the space race started, bringing with itself new design and analysis techniques such as Computer Based Design and the Finite Element Method [14], these achievements gave designers powerful tools to analyze complex structures and by the end of this decade one of the first Structural Analysis Software, NASTRAN (NASA Structural Analysis), was developed [11].

In the early 1970's, The Federal Aviation Administration, in the United States, introduced an amendment requiring the dynamic response of the aircraft to be evaluated for continuous turbulence in addition to the dynamic discrete gust [13]. Even before, there was an increasing concern in the engineering community about the amount of load cases required to determine the worst-case scenario for steady and dynamic loads [15]. Since a factorial search would be 
prohibitively expensive, two approaches were commonly used: mission analysis, based on the specific flight profile, in which the criteria for determining the limit loads was the frequency of exceedance at each mission segment; and the design envelope criterion, in which the worst load cases are found trough a point by point analysis [3, 16-18].

By the mid 1990's, The Loads and Dynamics Harmonization Working Group, to unify the airworthiness regulations around the globe, required the analysis of dynamic loads for multiple scenarios that were previously non-existent, such as emergency landing, ground maneuvers, tuned discrete gust, etc. [11,19]. Such historical development set the base for the current FAR-25 and CS-25 airworthiness requirements, which define the sheer number of load cases that must be analyzed to certify an airframe structure for commercial transport $[4,5,20]$.

With the increasing complexity of the computer models and the wide number of loads cases needed to be analyzed, researchers pursue new ways to simplify the estimation of aircraft loads. For instance, reduction methods such as the Guyan Reduction, Craig-Brampton, and the ComponentMode Synthesis [21] were further developed to reduce the number of degrees of freedom of the structural model, but still capture the underlying behaviour of the system.

The current state-of-the-art structural design optimization process is tightly bounded to its loads analysis. These concepts were matured during the 1970's and through the 1990's where several structural optimization surveys can be found $[14,22-26]$ that discussed the common problem of altering the dynamic behaviour of a structure while seeking weight minimization.

To alleviate some of these issues, constraints such as frequency range, displacement and stresses were sometimes imposed in the algorithms to tailor the structure's dynamic response [24,27-29]. Another approach was to specify a certain amount of material to attain a specific natural frequency 
[14]. These approaches rely heavily on a deterministic set of dynamic properties and could often lead to conservative and even off-optimal-performance designs.

In the specific case of dynamic aeroelastic loads, several efforts have been published in the past to account for the variation of the critical loads or to find the after-optimization critical loading condition in an efficient and accurate manner. Evaluating the worst loading condition due to turbulence has been one of the most discussed topics in the loads area due to the stochastic and uncertain nature of the physical phenomena $[3,12,30-33]$. It is also the most common numerical example in literature, however, unless explicitly stated, none of the techniques presented below are exclusive to gust related excitations.

The first efforts were recorded as early as 1980 with the introduction of the Statical Discrete Gust (SDG) $[18,30]$. The SDG approach aimed to reconcile the two most common approaches in modelling atmospheric excitations, Discrete Gust (1 Minus Cosine function) in the time domain and Continuous Turbulence (Von Karman Power Spectral Density function) in the frequency domain, by systematically searching over a specified family of equi-probable gust patterns the worst gust loading condition. Later, in 1991 The Matched Filter Theory [34-36] appeared, where the discrete tuned gust that would produce the maximum response in the system was calculated based on the principle of the convolution integral. The systems response to a unit impulse was calculated and normalized with respect to its own energy content. It was then reversed in time to obtain the "matched" or tuned input signal that would produce the maximum response.

A classical approach, though computationally expensive, is to estimate sensitivity derivatives. This methodology was used in the 90's, where, in some instances, the mode shapes were considered constant through the optimization to simplify the calculation [9, 37-39]. 
Reanalysis techniques were adopted recently in the field of Aeronautics [40-41] though these have been extensively studied in the field of civil engineering [42-47]. They employ the solution of the original structure and re-compute only the variation of the response. Following this approach, the magnitude of the deterministic critical loads selected during the design space exploration is updated as the structures parameters vary. However, the set of critical loads remain unaltered.

The airplane's dynamic behaviour is commonly modeled as a second order frequency domain formulation. Alternatively, these equations can be transformed into a Linear Time Invariant (LTI) state space representation and solved numerically in the time domain. This led various researchers to explore the use of the Lyapunov equation [32,48] and an energy-to-peak gain model [49] to determine the worst gust excitation of a 3D Finite Element model.

More recently, surrogate models, such as Kriging, Surface Response, and Second Order Regression, have gained popularity [50-52]. These are refined interpolation methods that approximate the behavior of complex responses based on a set of sample points, which are normally selected via Design of Experiment methodologies, and provide a computationally inexpensive way of exploring large design spaces [51-53]. For example, Kodaparast et. al. [41,54] presented an efficient algorithm based on the Kriging predictor to evaluate the worst gust load case. In this case, a Kriging predictor is built for each load at each station (e.g., the out of plane bending moment at the wing root) and monitored to find the gust wavelength that would produce the maximum dynamic response. Following this approach at an industrial level, the computational burden may be of the same size as the regular procedure because of the large number of loads and stations that are still required to be monitored. 
To improve such a drawback, Ghosh et. al. [55] proposed a Principal Component Analysis Assisted Surrogate Model to estimate the design loads using an orthogonal decomposition to un-correlate the loads to diminish the number of surrogate models needed to evaluate the worst loading condition. Following a similar approach, Tartaruga et al. [56] used the Singular Value Decomposition (SVD) method to reduce the order of the model in conjunction with a surrogate predictor. Castellani et al. [57] studied a Parametric ROM, in which the equations of motion were cast in the form of state space variables, sampled at different points in the flight envelope, reduced through matrix projection and interpolated through out the rest of the flight envelope, yielding accurate results with considerable computational savings.

Following the same approach as Kodaparast et. al., Ghosh et. al., and Tartaturga et. al. [41,54-56], in this thesis, the use of metamodeling techniques for the identification of critical loads after structural modifications are expanded. To that end, the Efficient Global Optimization (EGO) subalgorithm, as introduced by Jones et. al. [10] is implemented.

This sub-algorithm can be seen as an extension to the Kriging, in which a new sample points are added, until a certain level of accuracy has been achieved. In this way, the EGO allows to properly identify the global minimum/maximum points in the selected design space.

The issue of constructing an individual predictor for each load type at each station, as indicated by Kodaparast et. al. [41,54] is addressed by maximizing the use of the data extracted at each sampled point, exploiting similarities between families of curves.

Furthermore, considering that not every iteration of the structural optimization process will forcefully induce a significant change in the load(s), re-exploring the design space with every iteration might result in a wasted computational power. Instead, the use of a correlation model 
between a metric (based on the concept of $\mathrm{MCF}$ ) and the monitored load(s) at a single sampled point can be used to draw global information about the variation of the load(s). Acting as triggers for the re-exploration of the design space.

\subsection{THESIS OVERVIEW}

This thesis is composed of seven chapters. For the reader's convenience, the theoretical framework has been divided into three chapters, namely chapters 2,3 and 4 . Chapter 2 describes the most upto-date methods used in the current industrial process to estimate the aeroelastic loads. This background information is intended to give a non-expert reader a basic understanding of the complexity of the current methods and to identify some of the challenges that lie in the path of an integrated multidisciplinary design optimization platform.

Chapter 3 shows the mathematical development of the techniques that build up the EGO algorithm, which are: The Latin Hypercube sampling scheme, the Kriging model, the Model CrossValidation, and the Expected Improvement function. These methods are used jointly to interpolate, with a high degree of accuracy, design spaces that are computationally expensive to evaluate.

In chapter 4, different metrics, based on the concept of modal contribution factors, are reviewed to determine which one may be suitable to indicate (or flag) when a significant change in the magnitude of the critical load has occurred. The results of this analysis are also provided in this chapter, where a case study with a simple model composed of five degrees of freedom is used as a proof of concept and to select the metric that will be used in the Surrogate-Aided Response algorithm. 
Chapter 5 discusses the final implementation of the Surrogate-Aided Response block and in chapter 6 a case study based on the aeroelastic model of a Bombardier Aerospace platform is used to assess the performance of the method proposed. Finally, chapter 7 presents the conclusions where the advantages of the proposed method are discussed and some practical recommendations the user should keep in mind are given. Future research is also addressed in this last chapter. 


\section{THEORETICAL FRAMEWORK PART I: NUMERICAL MODEL FOR FLEXIBLE AIRCRAFT}

Modern transport aircraft structures are highly flexible. The mutual interaction between aerodynamic, elastic, and inertial forces play an important role in the structural integrity and the overall performance of the airplane. This interaction induces additional forces that redistribute the loading experienced by each structural component. The correct estimation of the aeroelastic loads is paramount to design reliable and optimal structures [58].

The aircraft free-free model, in the context of dynamic aeroelastic analysis, is comprised of rigid body and flexible degrees of freedom (DoF), where the structure's dynamic response is evaluated following a modal approach. The high-fidelity Detail Finite Element Model (DFEM) is replaced with a low-fidelity Stick-Model, while the aerodynamic high-fidelity Computational Fluid Dynamics (CFD) simulation is replaced by a low-fidelity panel method. The masses (structural and non-structural) are lumped throughout the main flexural axis of the structure and added independently to decouple the weight prediction from the structural model. Then the models are superimposed.

The coordinate system of the aerodynamic model commonly does not agree with the structural models' coordinate system, in which case an interpolation function is used to relate the forces and displacements in both coordinate systems.

These models and methods are accurate but simple enough to be called thousands of times during the aeroelastic loads analysis. In the following subsections, the mathematical tools typically used to estimate the loads for linear flexible aircraft are introduced. Additionally, the main models used 
to simulate atmospheric disturbances are also presented, these are: the one-minus-cosine gust, the sharp-edge gust and the continuous turbulence model.

\subsection{THE STRUCTURAL MODEL}

Aircraft are highly complex structures comprised of thousands of components that interact with each other. High fidelity representations such as a Detailed Finite Element Model (DFEM) are preferred for the optimization of local components. However, imposing such a large model for recurrent analysis is impractical. Therefore, the model is simplified firstly to a Global Finite Element Model (GFEM).

To capture the underlying dynamic behaviour of the system using a minimum quantity of DoF, the GFEM system is further transformed to a low-fidelity model known as the Stick-Model [59], as shown in Figure 2.1. In the following sections, a brief overview of these two structural models is presented.
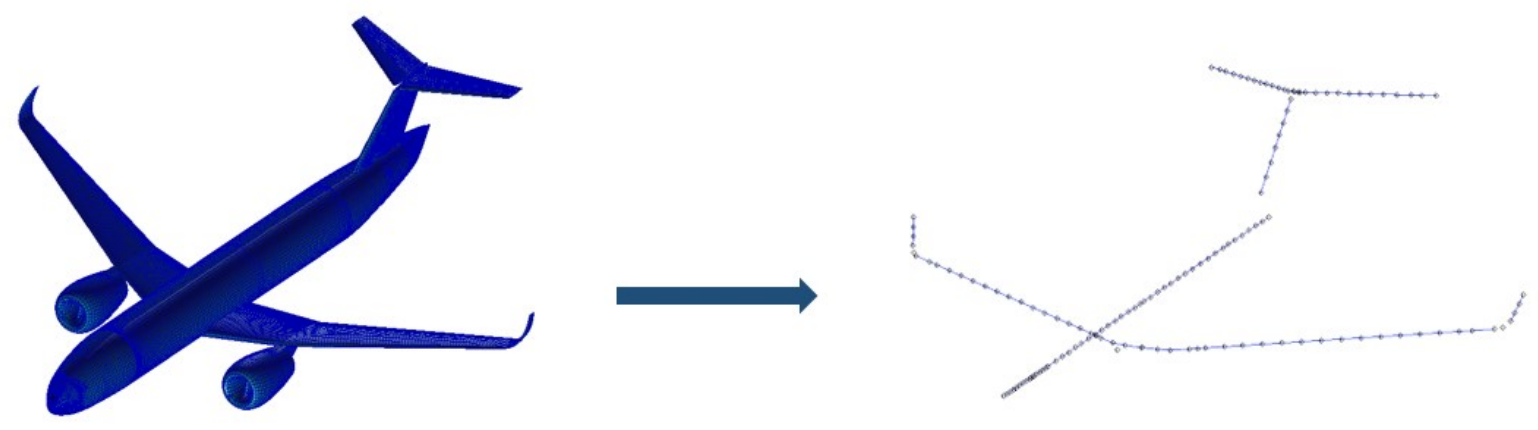

Figure 2.1. Visual representation of a GFEM (on the left) transformed to a low-fidelity model (on the right): The Stick-Model. 


\subsubsection{Global Finite Element Model}

The Finite Element method discretizes a complex structure into a number of elements of simple geometry connected through points known as nodes or grid points. In this thesis, the term grid is preferred. The properties of each of the elements are assembled to obtain the global mass and stiffness matrices of the structure, however, these models tend to be in the order of thousands of elements, making them unpractical for iterative analyses.

To further reduce the size of a GFEM, several methodologies exist in the literature [60]. However, in the framework of this thesis, the Stick Model is the only one being considered.

\subsubsection{Model Order Reduction - The Stick Model}

Once the structural layout is defined and the GFEM of the airframe is available, the Stick Model can be developed. The Stick Model is low-fidelity Finite Element Model consisting of a set of beam elements with equivalent axial and shear stiffness extending along the structures elastic axis. The method assumes that the stiffness properties are extracted with respect to the principal axes of the original geometry [61].

First, a local Cartesian coordinate system is created at the shear center of each wing-box station and it is oriented along the elastic axis as shown in Figure 2.2. The location of each wing station, $y_{j}^{*}$, is normalized with respect to the semi-span, $S^{*}$, as per equation 2.1 , to obtain the normalized length $\left(\eta_{j}-\eta_{i}\right)$ between two consecutive wing stations.

To obtain the displacements in the principal axis of each station, the 3 DoF associated with rotation are constrained, leaving only the 3 translational DoF free, in each of the skin-stringers grid points. 
Then the grids of the skin-stringers are connected to a grid point located at the shear center of the wing station with a rigid element, such as the RBE2 in NASTRAN, as can be seen in Figure 2.2.

A unitary load is applied at the shear center grid point of one of the two stations in each one of the principal directions $(\mathrm{x}, \mathrm{y}$, and $\mathrm{z})$ and the displacements $\left(d_{x}, d_{y}\right.$, and $\left.d_{z}\right)$ at both stations are retrieved. Then, the equivalent cross sectional area, $(A)_{i-j}$, is computed using equation 2.2, similarly the shear factors $\left(k_{z}\right)_{i-j}$ and $\left(k_{x}\right)_{i-j}$ are computed using equations 2.3 and 2.4.

In a similar fashion, a unitary moment is applied at the shear center grid point of one of the two stations about each one of the principal directions $(\mathrm{x}, \mathrm{y}$, and $\mathrm{z})$ and the angular displacements $\left(\theta_{x}, \theta_{y}\right.$, and $\left.\theta_{z}\right)$ at both stations are retrieved. The second moments of area, and the second polar moment of area can be estimated using equations 2.5-2.7.

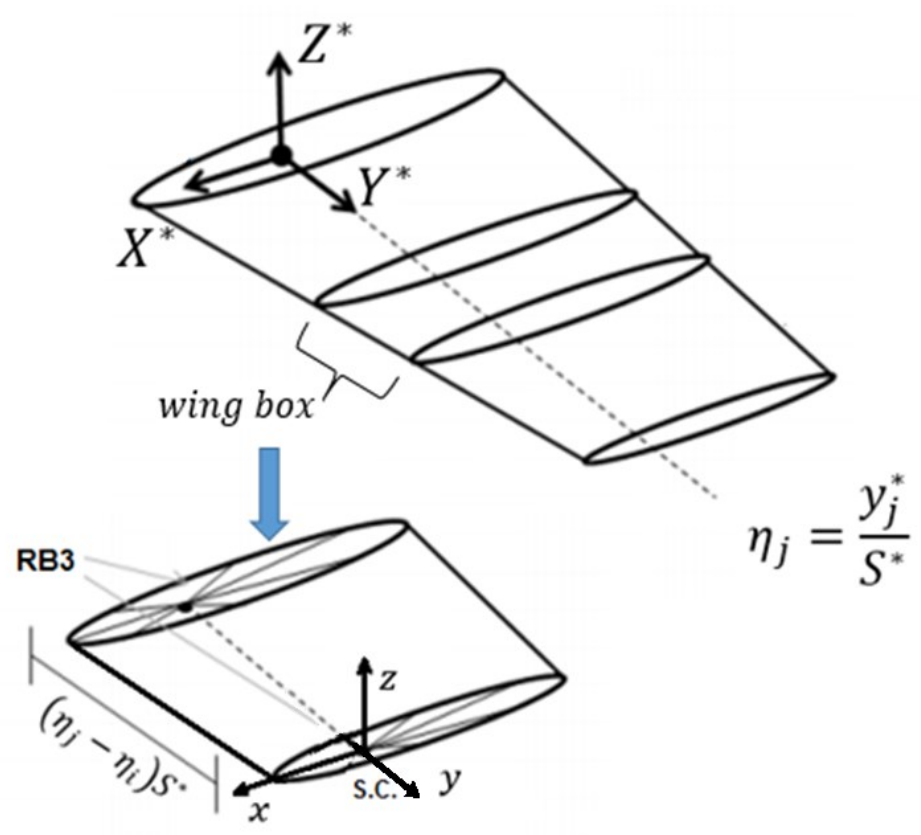

Figure 2.2. Wing box axes used to extract the stiffness properties 


$$
\begin{gathered}
\eta_{j}=\frac{y_{j}^{*}}{S^{*}} \\
(A)_{i-j}=\frac{1}{E}\left|\frac{\left(\eta_{j}-\eta_{i}\right) S^{*}}{\left(d_{y_{j}}-d_{y_{i}}\right)}\right| \\
\left(k_{x}\right)_{i-j}=\frac{1}{G A}\left|\frac{\left(\eta_{j}-\eta_{i}\right) S^{*}}{\left(d_{x_{j}}-d_{x_{i}}\right)}\right| \\
\left(k_{z}\right)_{i-j}=\frac{1}{G A}\left|\frac{\left(\eta_{j}-\eta_{i}\right) S^{*}}{\left(d_{z_{j}}-d_{z_{i}}\right)}\right| \\
\left(\mathrm{I}_{x}\right)_{i-j}=\frac{1}{E}\left|\frac{\left(\eta_{j}-\eta_{i}\right) S^{*}}{\left(\theta_{x_{j}}-\theta_{x_{i}}\right)}\right| \\
\left(\mathrm{I}_{z}\right)_{i-j}=\frac{1}{E}\left|\frac{\left(\eta_{j}-\eta_{i}\right) S^{*}}{\left(\theta_{z_{j}}-\theta_{z_{i}}\right)}\right| \\
\left(\mathrm{I}_{y}\right)_{i-j}=\frac{1}{G}\left|\frac{\left(\eta_{j}-\eta_{i}\right) S^{*}}{\left(\theta_{y_{j}}-\theta_{y_{i}}\right)}\right|
\end{gathered}
$$

The accuracy of this method relies on the assumption that the elastic axis and the principal torsional axis are coincident. However, the assumption is no longer valid when the wing possesses a dihedral angle, wing swept angle and wing twist, since the elastic axis will be tilted from the principal torsional axis [61]. 
Other methods have been proposed in the literature to condense the DoF's of complex structures, known as Model Order Reduction (MOR) techniques, such as the Guyan- Iron, the Dynamic reduction, or the Craig-Brampton [60,62]. However, these methods do not provide a physical representation of the aircraft and therefore the Stick-Model is still preferred.

\subsubsection{Modal analysis and the generalized coordinates}

The response $\{u(t)\}$ of any linear time invariant system may be expressed as a linear combination of harmonic motions, where each harmonic function $q_{i}(t)$ has a clear distinct deformation pattern, called a mode shape $\varphi_{i}$ and it is associated with a discrete frequency, known as a natural frequency $\omega_{n_{i}}[63]$

$$
\{u(t)\}=\sum_{i=1}^{m}\left\{\varphi_{i}\right\} \mathrm{q}_{i}(\mathrm{t})
$$

The natural frequencies and corresponding mode shapes can be obtained by solving the classical eigenvalue problem of an undamped free vibrating system.

$$
\left([K]-\omega_{n_{i}}^{2}[M]\right)\left\{\varphi_{i}\right\}=0
$$

To compute the solution numerically iterative methods are often used such as the Inverse Iteration Method or the Lanczos Method [64]. The principle lies in the fact that each eigenvector can be approximated as a linear combination of the other linearly independent eigenvectors (equation 2.10) and make use of the Rayleigh quotient (equation 2.11) to evaluate the corresponding eigenvalue, such that: 


$$
\begin{gathered}
\left\{\varphi_{i}^{k}\right\}=[K]^{-1}[M]\left\{\varphi_{i}^{k-1}\right\} \\
\omega_{n_{i}}^{2}=\frac{\left\{\varphi_{i}\right\}^{T}[K]\left\{\varphi_{i}\right\}}{\left\{\varphi_{i}\right\}^{T}[M]\left\{\varphi_{i}\right\}}
\end{gathered}
$$

The solution of eigenvalues and eigenvectors is often an expensive computational problem when dealing with structures with thousands of DoF, since there will be as many eigenvalues and eigenvectors as DoF. The method selected for the extraction of eigenvalues and eigenvectors strongly depend on the frequency range of interest and the number of massless degrees of freedom associated with the model. The theoretical development of such methods falls outside of the scope of this project. However, Saad, Y. [65] provides a large overview of such iterative methods and MacNeal, R. [64] provides brief guidelines for selecting the best method for the intended application.

To simplify the solution of the equations of motion, the eigenvectors are used to uncouple the stiffness, mass and in some cases the damping matrices (i.e., when proportional damping is assumed). Since the modes are orthogonal, the off-diagonal terms become zero and the new diagonal matrices are called modal mass $M_{q}$, and modal stiffness $K_{q}$.

$$
\begin{gathered}
{\left[M_{q}\right]=\left\{\varphi_{i}\right\}^{T}[M]\left\{\varphi_{j}\right\}=\left\{\begin{array}{cc}
0, & i \neq j \\
m_{q_{j}}, & i=j
\end{array}\right.} \\
{\left[K_{q}\right]=\left\{\varphi_{i}\right\}^{T}[K]\left\{\varphi_{j}\right\}=\left\{\begin{array}{cc}
0, & i \neq j \\
k_{q_{j}}, & i=j
\end{array}\right.}
\end{gathered}
$$

The harmonic functions defined in equation 2.8 are also known as the modal coordinates or generalized coordinates and are used to simplify the solution of flexible aircraft equations of motion. 


\subsection{THE AERODYNAMIC MODEL}

High-fidelity CFD based on Navier-Stokes equations are prohibitively expensive for the loads

analysis due to the sheer number of load cases that are needed to determine the critical loads. Instead, low-fidelity models based on potential flow theory are employed [7].

There are several methods found in the literature, such as Strip Theory, the Doublet-Lattice Method, or the Vortex-Lattice Method that provide good approximations for the distribution of the aerodynamic forces. However, if the analysis lies in the transonic flight regime the models should be corrected based on wind tunnel test data or a set of high-fidelity simulations [6]. In more recent years, supersonic low-fidelity models such as the ZONA 51 [64] have been implemented in legacy codes such as Nastran.

In the present work, the Doublet-Lattice Panel Method will be used to determine unsteady aerodynamic influence coefficients (AIC's) in the frequency domain. The AIC's can be tightly coupled with the FEM method and represent an inexpensive way to compute the distribution of the aerodynamic forces over arbitrary lifting surfaces.

\subsubsection{The Doublet-Lattice panel method}

The Doublet-Lattice Panel method is based on linearized aerodynamic potential flow theory, where the flow may be steady or unsteady (harmonically oscillating) [64]. The external configuration of the aircraft is divided into trapezoidal panels, which are further subdivided into lifting elements, also called boxes, where an acceleration potential doublet of uniform but unknown strength is located at each lifting element quarter chord [66] as depicted in Figure 2.3. 
The set of control points $(j$ set) is used to compute the downwash vectors and are located at each lifting element three-quarter chord, where the Kutta condition of flow tangency must be met [66]. The aerodynamic grid points $(k s e t)$ are physically located at the center of each lifting element where forces and moments due to the pressure distribution are calculated.

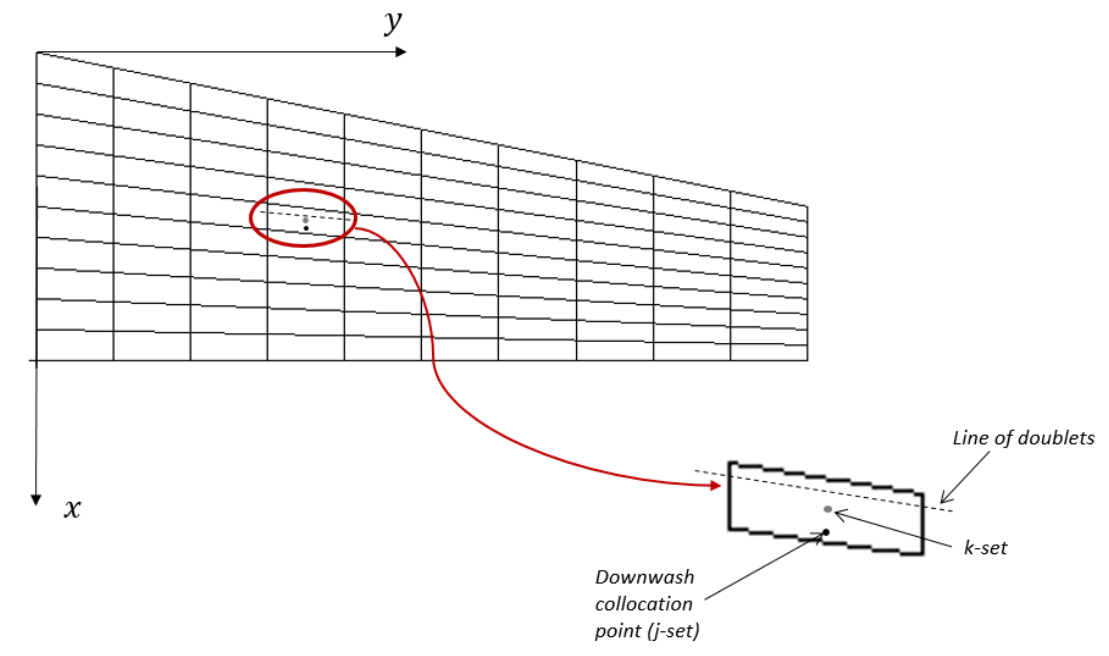

Figure 2.3. Doublet-Lattice Aerodynamic Panel.

The matrix $A_{j j}$ is known as the Aerodynamic Influence Coefficient (AIC's) matrix and it is evaluated at the control points $(j-s e t)$, it relates the dimensionless induced normal velocity, $w_{j}$, to the normalized lifting pressure on each lifting element,$\left\{f_{j} / \bar{q}\right\}$.

$$
\left\{w_{j}\right\}=\left[A_{j j}\right]\left\{f_{j} / \bar{q}\right\}
$$

A second relationship between the substantial differentiation matrices $\left(D_{j k}^{1}\right.$ and $\left.D_{j k}^{2}\right)$ and the aerodynamic grid points ( $k$-set) displacements is used to compute the downwash at the $j$-th point due to the $k$-th displacement. 


$$
\left\{w_{j}\right\}=\left[D_{j k}^{1}+i k D_{j k}^{2}\right]\left\{u_{k}\right\}+\left\{w_{j}^{g}\right\}
$$

The component $w_{j}^{g}$ accounts for the static downwash that may arise from an initial condition, such as camber, wing twist or initial angle of attack.

The forces acting at the aerodynamic grid points $\left\{P_{k}\right\}$ are computed by integrating the pressure of each lifting element that comprise the panel:

$$
\left\{P_{k}\right\}=\left[S_{k j}\right]\left\{f_{j}\right\}
$$

where $\left[S_{k j}\right]$ is the integration matrix. Finally, by combining equations $2.14-2.16$ the Force Aerodynamic Influence Coefficients matrix $\left[Q_{k k}\right]$ evaluated at the aerodynamic grid points can be computed as follows:

$$
\left[Q_{k k}\right]=\left[S_{k j}\right]\left[A_{j j}\right]^{-1}\left[D_{j k}^{1}+i k D_{j k}^{2}\right]
$$

It should be pointed out, that the AIC's are a function of the Mach number and the reduced frequency $k$, where the reduced frequency is defined as:

$$
k=\frac{\omega b}{V_{\infty}}
$$

and where $V_{\infty}$ is the free stream velocity and $b$ is the semi-chord. Since there is a dependency between the AIC's and the circular frequency of oscillation, the loads analysis is conventionally carried out in the frequency domain and transformed back into the time domain using Fourier transformations. However, there are different approaches to approximate the values of AIC in the time domain. One such approach is by fitting Rational Approximation Functions with a least squares method [7]. 


\subsection{SPLINING INTERPOLATION - TRANSFER OF AERODYNAMIC FORCES TO THE STRUCTURAL GRID}

The aerodynamic grid set is created independently from the structural grid set and generally this two sets do not coincide. To transfer the loads and displacements from one set to the other, an interpolation function is needed, which is referred to as splining.

The splining matrix $G_{k g}$ relates the aerodynamic displacements in the $k$-set to the structural degrees of freedom, namely the $g$-set, as:

$$
\left\{u_{k}\right\}=\left[G_{k g}\right]\left\{u_{g}\right\}
$$

The two system of forces should equally deform the structure, so that based on the principle of virtual work, the virtual displacements from the aerodynamic grid, $\delta u_{k}$, should produce the same virtual work as the virtual displacements from the structural grid, $\delta u_{g}$, set, thus:

$$
\left\{\delta u_{k}\right\}^{T}\left\{F_{k}\right\}=\left\{\delta u_{g}\right\}^{T}\left\{F_{g}\right\}
$$

Substituting equation 2.19 into equation 2.20 yields equation 2.21 .

$$
\left\{\delta u_{g}\right\}^{T}\left(\left[G_{k g}\right]^{T}\left\{F_{k}\right\}-\left\{F_{g}\right\}\right)=0
$$

The transfer of aerodynamic loads to the structural grid points is then given by:

$$
\left\{F_{g}\right\}=\left[G_{k g}\right]^{T}\left\{F_{k}\right\}
$$

The splining, $G_{k g}$, matrix can be derived using different techniques, such as linear splines or surface splines. The surface spline is based on classic infinite plate theory, whereas the linear spline is a generalization of an infinite beam, in which the infinite beam possesses a set of known deflections and twist angles [64]. The derivation of the surface splining matrix is beyond the scope 
of this document; however, any interested reader is referred to Harder, R.L. \& Desmarais, R.N. [67], in which a detailed description of their mathematical development is given.

Using the splining matrix, the force AIC's which provides the variation of aerodynamic forces due to structural deformations evaluated in the structural grid is found as follows:

$$
\left[Q_{g g}\right]=\left[G_{k g}\right]^{T}\left[Q_{k k}\right]\left[G_{k g}\right]=\left[G_{k g}\right]^{T}\left[S_{k j}\right]\left[A_{j j}\right]^{-1}\left[D_{j k}^{1}+i k D_{j k}^{2}\right]\left[G_{k g}\right]
$$

A further modal reduction is needed to perform a dynamic aeroelastic analysis. Similar to equations 2.12 and 2.13, for the generalized mass and stiffness matrices, the generalized force AIC's matrix in the modal coordinate system is computed using the following expression.

$$
\left[Q_{q}\right]=\left[\varphi_{i}\right]^{T}\left[Q_{g g}\right]\left[\varphi_{i}\right]
$$

\subsection{THE AEROELASTIC EQUATION IN MODAL COORDINATES}

The classic form of the aeroelastic equations of motion with reduced frequency dependent aerodynamics in forced vibration and expressed in the frequency domain, is given by equation 2.25. This expression is evaluated with respect to a steady initial position ( $1 \mathrm{~g}$ level flight) where a small disturbance $\{P(\omega)\}$ excites the structure.

$$
\left[-M_{q} \omega^{2}+i C_{q} \omega+K_{q}-\frac{1}{2} \rho V_{\infty}^{2} Q_{q}(m, k)\right]\{q(\omega)\}=\{P(\omega)\}
$$

Here, $\{q(\omega)\}$ is the vector of generalized (or modal) coordinates, $P(\omega), M_{q}, C_{q}, K_{q}$ and $Q_{q}(m, k)$ are the generalized forcing function, the generalized or modal mass, modal damping, modal stiffness, and modal aerodynamic influence coefficients, respectively. 
The generalized forcing function in the frequency domain, $P(\omega)$, is obtained by transforming the time domain functions via Fourier transform, as in equation 2.26.

$$
\{P(\omega)\}=\int_{-\infty}^{\infty}\{P(t)\} e^{-i \omega t} d t
$$

where $\{P(t)\}$ is the generalized forcing function vector in the time domain. The generalized forcing function vector may contain generalized non-aerodynamic forces, $\left\{P_{g e n}(t)\right\}$, and generalized aerodynamic disturbances, $\left\{P_{\text {aero }}(t)\right\}$ as per equation 2.27 .

$$
\{P(t)\}=\left\{P_{\text {gen }}(t)\right\}+\left\{P_{\text {aero }}(t)\right\}
$$

The non-aerodynamic forces are applied directly on the structural grid points of the Stick-Model $(g$-set). Therefore, their transformation from the physical coordinates to the generalized (or modal) coordinates is straight forward as:

$$
\left\{P_{g e n}(t)\right\}=\left[\varphi_{i}\right]^{T} F(t)
$$

where $F(t)$ is the vector of non-aerodynamic forces. For the aerodynamic forces to be transformed to the generalized coordinates four relationships are required. Firstly, to express the forces in the aerodynamic grid points $(k$-set) induced by a downwash applied at any aerodynamic box control point $(j-s e t)$ as in equation 2.29 .

$$
\left[Q_{k j}\right]=\left[S_{k j}\right]\left[A_{j j}\right]
$$

Secondly, to express the aerodynamic forces in the $k$-set to the structural grid points $(g$-set $)$ through the splining matrix: 


$$
\left[Q_{g j}\right]=\left[G_{k g}\right]^{T}\left[Q_{k j}\right]
$$

Thirdly, to transform the matrix of aerodynamic forces applied in the structural grid ( $g$-set) due to a downwash applied in the $j$-set, $\left[Q_{g j}\right]$, to the generalize coordinates:

$$
\left[Q_{q j}\right]=\left[\varphi_{i}\right]^{T}\left[Q_{g j}\right]
$$

And finally to relate the downwash forcing function $\left\{w_{j}(t)\right\}$ to the matrix of generalized aerodynamic forces due to a downwash vector at any collocation point $\left(\left[Q_{q j}\right]\right)$ :

$$
\left\{P_{\text {aero }}(t)\right\}=\bar{q}\left[Q_{q j}\right]\left\{w_{j}(t)\right\}
$$

In the following subsection we will provide the most common functions used to model the downwash forcing function $\left\{w_{j}(t)\right\}$. In practice, these are: the one-minus cosine function in the time domain and the Von-Karman spectrum given as a function in the frequency domain. The sharp-edge gust is also discussed for the sake of completeness; however, this function is no longer used in practice.

The modal aerodynamic influence coefficients will be real numbers if the analysis involves quasisteady aerodynamics. However, if the analysis is required to account for the effects of unsteady aerodynamics (as in the case of dynamic aeroelastic problems) the coefficients will be complex numbers [3].

The generalized coordinates contain elastic as well as rigid body modes. The latter are characterized by having a zero-natural frequency. In the rigid body modes all the lumped masses of the structure are oscillating in phase and there is no stress induced within the structure [6]. 
The solution of the modal coordinates can be obtained in terms of a transfer function, $H(\omega)$, which is simply the inverse of the term in brackets of equation 2.25 , such that:

$$
\{q(\omega)\}=\frac{1}{\left[-M_{q} \omega^{2}+i C_{q} \omega+K_{q}-\frac{1}{2} \rho V_{\infty}^{2} Q_{q}(m, k)\right]}\{P(\omega)\}=[H(\omega)]\{P(\omega)\}
$$

The aeroelastic response of the structure to an arbitrary excitation can be calculated through equation 2.33 by a direct multiplication between the transfer function and the excitation in the frequency domain. To obtain the response in the time domain, an Inverse Fourier Transform is carried out in the form of equation 2.34 .

$$
\{q(t)\}=\int_{-\infty}^{\infty}[H(\omega)]\{P(\omega)\} e^{i \omega t} d \omega
$$

\subsection{ATMOSPHERIC DISTURBANCES FOR COMMERCIAL TRANSPORT AIRCRAFT}

Regulatory agencies as well as manufacturers' policies determine the minimum requirements to assess structural integrity. Throughout this section, some important definitions regarding atmospheric disturbances are introduced, based on sections FAR 25.301-25.537 [5].

To ensure that the maximum loads for each station in the airplane are estimated, enough points on and within the boundaries of the design envelope must be investigated. The aircraft must be able to withstand any forced structural vibration, whether due to an operational condition, such as a gust encounter or due to a failure, malfunction, or adverse condition in the flight control system (unless shown to be extremely improbable) [5].

This implies that the strength of the airframe must be evaluated at the limit loads where no detrimental permanent deformation should be observed. The limit loads are the maximum external 
loads to be expected during service life. The ultimate loads correspond to the limit loads multiplied by the prescribed 1.5 safety factor where the structure must withstand the ultimate load for 3 seconds before failure.

The case of discrete gust and continuous turbulence will be explained in further detail in the next subsection, since they represent the most common types of external excitation the aircraft will experience in flight and among the most common numerical examples found in the literature.

\subsubsection{Gust and Turbulence models}

Gust and turbulence are disturbances in the atmosphere that modify the pressure distribution around the lifting surfaces inducing additional loads. When there is a net change in the effective angle of incidence due to a gust-induced velocity gradient normal to the flight path, the gust is said to be a vertical gust (it should be noted that lateral gust falls within the same description). When the gust-induced velocity gradient is parallel to the flight path it is called a head-on gust [3]. Through out this project, only the case of vertical gusts is addressed.

Due to the uncertain nature of the phenomena, engineers and scientist developed two approaches to model the behaviour of the atmosphere, based on the profile of the disturbance: the discrete gust approach and the continuous turbulence approach. Both are still in use today and procurement agencies often expect both analyses to be conducted for certification purposes $[4,5]$.

\subsubsection{Discrete Gusts}

As the name implies, the discrete gust is an isolated disturbance with a pulse type profile. The simplest approximation is the Sharp-Edge Gust and was replaced later within the JAR/FAR 
regulations by the so called one-minus cosine profile which attempts to represent more closely the velocity gradients observed in the atmosphere $[12,13]$.

\subsection{Sharp Edge Gust}

The simplest approximation of a vertical gust is a step function, normally called a sharp-edge gust. The penetration effects are not considered and the analysis is restricted to plunge rigid body motions only.

Consider an aircraft flying in a steady trimmed condition that encounters a sudden symmetrical vertical sharp-edge gust as depicted in Figure 2.4.
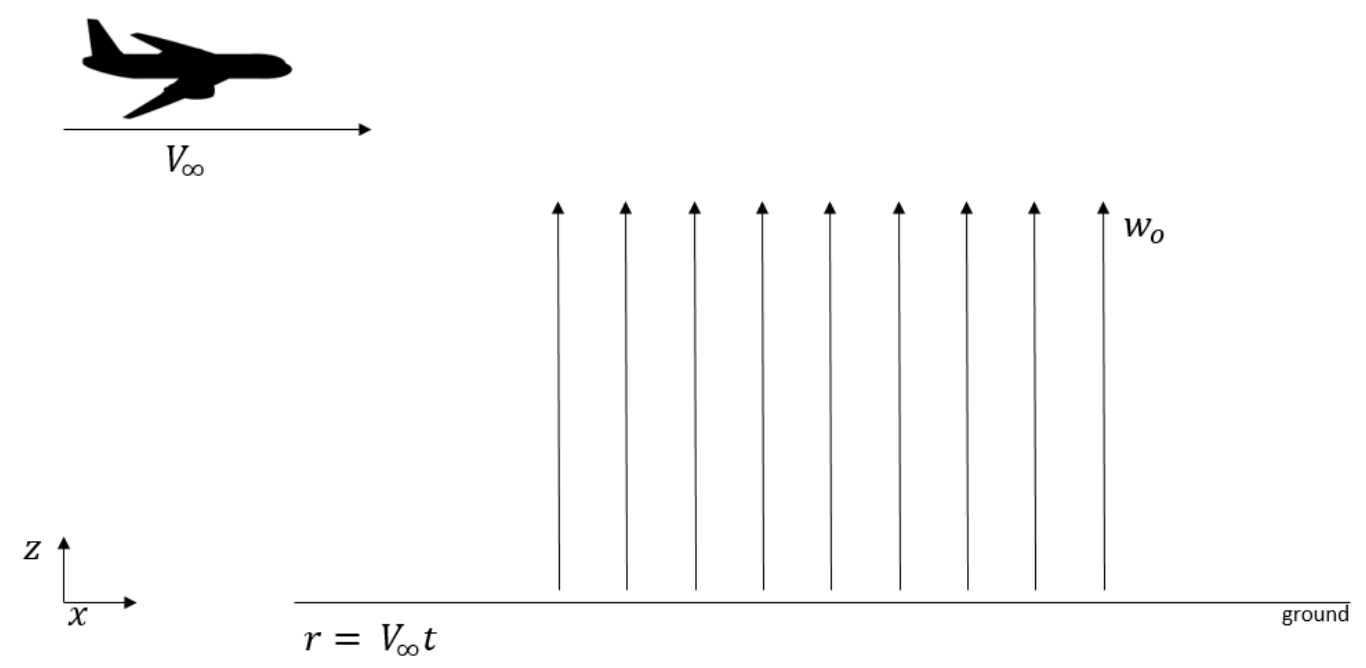

Figure 2.4. Sharp edge gust.

The incremental load factor as a function of wing loading is given by the following equation:

$$
\Delta \eta=\frac{\Delta L}{W}=K_{g} \frac{\rho}{2} \frac{V_{\infty} C_{L \propto} W_{g}}{W / S}
$$


where $\Delta L$ is the incremental lift, $W$ is the aircraft weight, $\rho$ is the air density, $C_{L \alpha}$ is the lift coefficient, $w_{g}$ is the gust velocity and $K_{g}$ is load alleviation factor. Here, the load alleviation factor, $K_{g}$, attempted to account for the unsteady aerodynamic effects. It was determined from empirical data collected from six different airplanes and normalized with respect to the Boeing B247 [13]. Then, the overall load factor, $\eta$, is calculated readily using Pratt's equation [13]:

$$
\eta=1+\Delta \eta=1+\frac{\rho}{2} \frac{V_{\infty} C_{L \alpha} w_{g}}{W / S} K_{g}
$$

Where $K_{g}$ is a function of the mass ratio, $\mu_{g}$, and is calculated as:

$$
K_{g}=\frac{0.88 \mu_{g}}{5.3+\mu_{g}}
$$

while the mass ratio, $\mu_{g}$, is computed as:

$$
\mu_{g}=\frac{2 W}{\rho g S c C_{L \propto}}
$$

where $g$ is the gravitational acceleration, $S$ is the wing area, and $c$ is the aerodynamic chord.

\subsection{One-Minus-Cosine Gust}

The gust velocity term, $w_{g}$, in equation 2.35 was replaced by a one-minus cosine function, as depicted in Figure 2.5, in 1964 within the JAR/FAR 25 regulations $[3,13]$. The revised criteria is still currently in force and states that gust loads must be determined by dynamic analysis and must consider all significant elastic and rigid DoF.

$$
w_{g}=\frac{w_{o}}{2}\left(1-\cos \frac{\pi r}{\lambda_{g}}\right)
$$


The one-minus cosine gust profile should be evaluated for gust gradients $\lambda_{g}$ ranging from 60 to 700 feet and the distance penetrated the gust, $r$, is evaluated from 0 to $2 \lambda_{g}$. The gust equivalent air speed, $w_{o}$, may be computed using the following expression:

$$
w_{o}=w_{r e f} F_{g}\left(\frac{\lambda_{g}}{350}\right)^{1 / 6}
$$
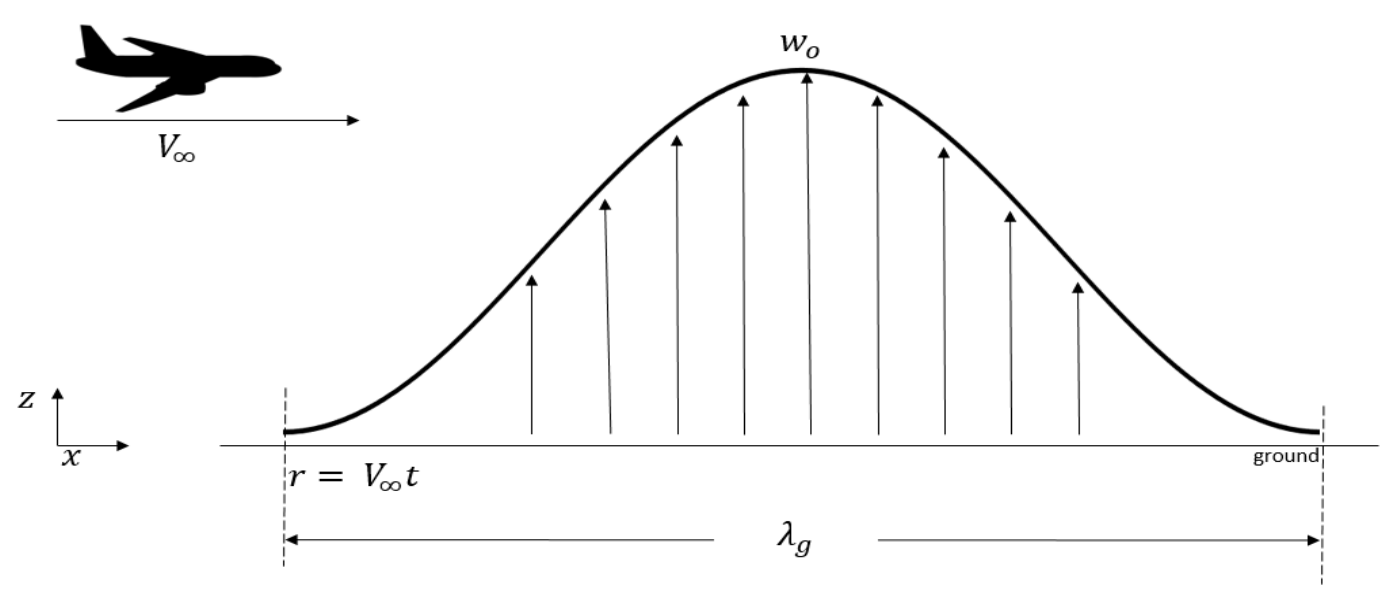

Figure 2.5. One-minus cosine gust.

where $w_{r e f}$ are prescribed values of gust equivalent air speeds dictated by the regulatory agencies and $F_{g}$ is the flight profile alleviation factor.

The profile alleviation factor, $F_{g}$, is increased linearly from sea level up to a value of 1.0 at the maximum operating altitude, $Z_{m o}$. The value of the profile alleviation factor at sea level is computed using equations 2.41 through 2.45 . 


$$
\begin{gathered}
R_{1}=\frac{\text { max landing weight }}{\text { max take off weight }} \\
R_{2}=\frac{\text { max zero }- \text { fuel weight }}{\text { max take off weight }} \\
F_{g m}=\sqrt{R_{2} \tan \left(\pi R_{1} / 4\right)} \\
F_{g z}=1-\frac{Z_{m o}}{250000} \\
F_{g}=0.5\left(F_{g z}+F_{g m}\right)
\end{gathered}
$$

\subsubsection{Continuous Turbulence}

The assumption of the idealized discrete gusts for loads analysis was a topic of great controversy during the 1950's and 1960's, since many scientists and engineers agreed that this idealization did not closely represented the behaviour of the atmosphere [12]. In 1962 Von Karman presented his "isotropic turbulence" spectrum which was included in the FAR 25 G appendix in 1970 in the amendment 25-23, which required the dynamic response of the aircraft to be evaluated for vertical and lateral continuous turbulence $[3,13]$.

Turbulence itself is regarded as a stochastic phenomenon, i.e. it is of apparent random nature. To model its behaviour, the profile is idealized as a Gaussian stationary random process, that is, it has an infinite duration and its statistical properties are the same everywhere as it is sampled [3].

The isotropic turbulence spectrum for vertical and lateral turbulence is in the form of equation 2.46 which can be written as: 


$$
\Phi(\Omega)=\left(\sigma_{w}^{2} l / \pi\right) \frac{1+\frac{8}{3}(1.339 l \Omega)^{2}}{\left[1+(1.399 l \Omega)^{2}\right]^{11 / 6}}
$$

where $\Phi(\Omega)$ is the gust power spectral density, $\Omega$ is the spatial frequency, $l$ is the scale of the turbulence and $\sigma_{w}^{2}$ is the square of the mean gust velocity. For heads-on turbulence, equation 2.47 is used instead which results in:

$$
\Phi(\Omega)=\left(\sigma_{w}^{2} / \pi\right) \frac{2 l}{\left[1+(1.399 l \Omega)^{2}\right]^{5 / 6}}
$$

Just as in the case of the discrete gust, the prescribed velocities are defined by each regulatory agency (FAR-25; CS-25). The scale of the turbulence, $l$, is another prescribed parameter, which has a value of $25000 \mathrm{ft}$. for civil aircraft gust loads estimations for all altitudes.

The spatial frequency, $\Omega$, also known as reduced frequency is computed as:

$$
\Omega=\frac{\omega}{V_{\infty}}
$$

where $\omega$ is the circular frequency and $V_{\infty}$ is the free stream velocity. Other reduced frequencies can be found in literature. Herein two reduced frequencies will be commonly used $\Omega$ and $k$. The relationship between both reduced frequencies is given by equation 2.49 , where $b$ is the aerofoil semi-chord.

$$
k=\frac{\omega b}{V_{\infty}}=\Omega \mathrm{b}
$$




\subsection{LoAd Recovery Methods}

Different methods have been proposed in the literature to recover the internal loads once the equations of motion have been solved in modal domain. In the following section, we will present a brief overview of the three most widely used methods, namely, the Mode Displacement Method, the Summation of Forces Method, and the Mode Acceleration Method [68].

\subsubsection{Mode displacement method}

In the Mode Displacement Method (MDM), the displacements using physical coordinates are retrieved using equation 2.8 (shown here below as equation 2.50), after transforming the frequency domain solution of equation 2.33 into the time domain using the Inverse Fourier transformation.

$$
\{u(t)\}=\sum_{i=1}^{m}\left\{\varphi_{i}\right\} q_{i}(t)
$$

The number of modes, $m$, used to retrieve the displacements, $\{u(t)\}$, is generally much smaller than the overall number of degrees of freedom of the system, $N$, since higher frequency modes are truncated at the frequency range of interest [68].

The set of bending and torsional moments and shear forces $\left\{\mathrm{R}_{e}(\mathrm{t})\right\}$, at each element is then calculated by:

$$
\left\{\mathrm{R}_{e}(\mathrm{t})\right\}=\left[C^{T}\right]\left[\mathrm{K}_{e}\right] \sum_{i=1}^{m}\left\{\varphi_{j, i}\right\} q_{i}(t)
$$


where $\left[\mathrm{K}_{e}\right]$ is the stiffness matrix of each element in the global coordinate system, $\left\{\varphi_{j, i}\right\}$ is the rows of the eigenvector matrix associated with the element $e$, and $C^{T}$ is a rotational matrix to transform the loads from the global coordinate system to the element coordinate system.

\subsubsection{Summation of forces method}

In the Summation of Force Method, the displacements due to inertial, elastic, and aerodynamic forces are also computed using equation 2.50. Thus, they are computed using the matrix of truncated eigenvectors.

To improve the quality of the results equation 2.52 is used. Here the response can be seen as a pseudo-static response, where the summation of the inertial, elastic and aerodynamic terms act as dynamic correction when a pseudo-static force, $\{P(t)\}$, is applied to the structure.

$$
\{u(t)\}=[K]^{-1}\{P(t)\}-\sum_{i=1}^{m}\left\{\varphi_{i}\right\} q_{i}(t)
$$

The loads can then be recovered as per the following equation:

$$
\left\{\mathrm{R}_{e}(\mathrm{t})\right\}=\left[C^{T}\right]\left[\mathrm{K}_{e}\right]\left\{u_{e}(t)\right\}
$$

where $u_{e}(t)$ are the displacements associated with the grid points of such an element $e$ calculated using equation 2.52 .

\subsubsection{Selection of critical loads and the loads envelope}

Once the loads have been recovered at each wing element for several cases investigated, the determination of the critical peak single loads and the critical correlated loads becomes an 
extensive endeavour to localize the minimum and maximum values that will serve for both, certification and optimization.

\subsubsection{Single loads envelope}

The time history response for each load at each station is obtained as a result of the loads recovery, where the maximum and minimum responses can be easily identified, as seen in Figure 2.6. (A). However, this representation is impractical considering the amount of stations and cases to be analyzed.

Instead, a 1-D load envelope is constructed by plotting the maximum and minimum response for each case investigated. This representation allows for visualization of the distribution of the $\max / \mathrm{min}$ forces and moments along a component as shown in Figure 2.6. (B) [69].

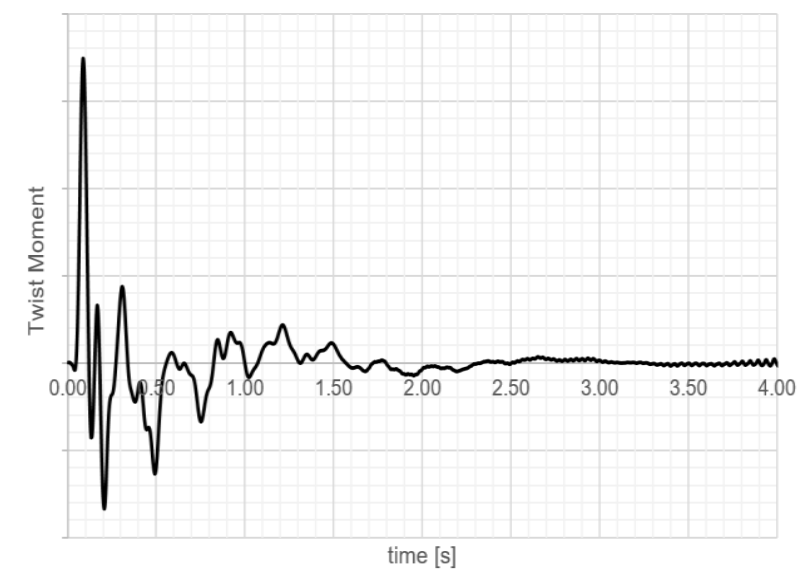

(A)

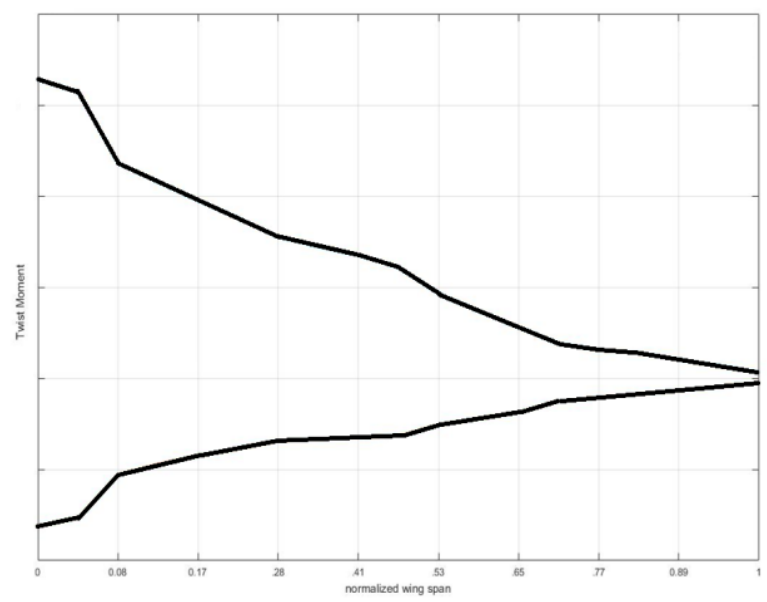

(B)

Figure 2.6. A) Time history of the twist moment. B) 1-D load envelope: twist moment along the wing. 


\subsubsection{Correlated loads}

The correlated loads can be found by plotting a specific set of loads in what is called an egg-plot, for example the bending moment versus torque at a given station of the aircraft, as seen in Figure 2.7. Each point represents a load case from which the bending and torque moments where computed and the points at the top of the scatter represent the critical correlated loads. A convex hull algorithm can also be employed through time dependant loads (such as in the case of gust loads) to obtain the corresponding convex hull [55].

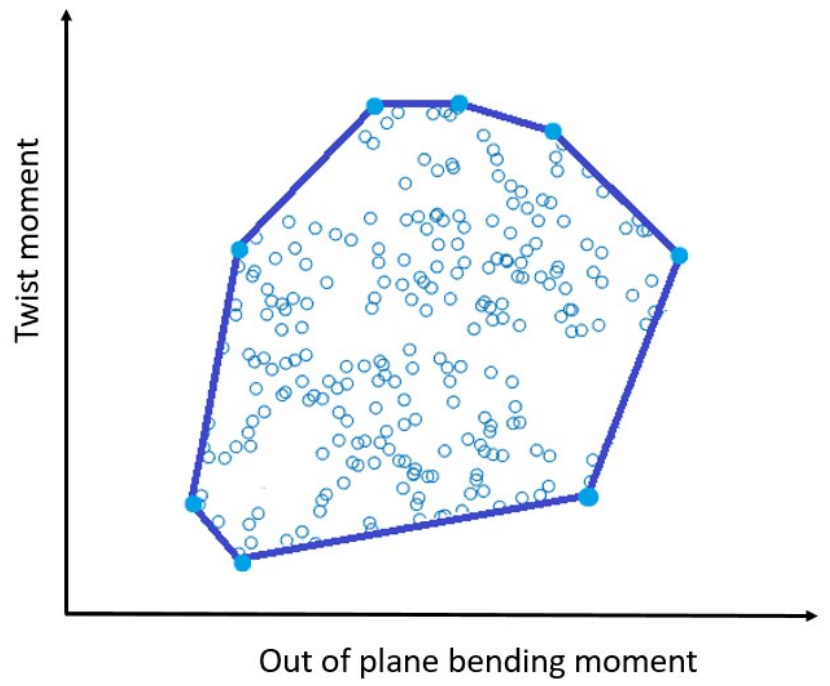

Figure 2.7. Convex hull of correlated loads of a commercial transport aircraft at the wing root. 


\section{THEORETICAL FRAMEWORK PART II: EFFICIENT GLOBAL OPTIMIZATION (EGO)}

So far, the mathematical models of the physical system have been described, its disturbances and methods to compute its response. Throughout this chapter, the theory behind one of the two main blocks, presented earlier in Figure 1.5, that comprise the "Surrogate-Aided Response" algorithm is described in detail.

The EGO algorithm, as presented in reference [10], consists mainly of 4 steps:

1. Initial selection of sampling points

2. Initial estimation of the surface response

3. Diagnosis (Model - Cross Validation)

4. Selection of new data points based on the Expected Improvement Function

The initial sample points can be chosen via random selection or following a more complex Design of Experiments method, here the Latin Hypercube scheme was used. Then, an initial surface response is computed using the Kriging metamodeling technique and a cross validation test is performed to assess if the model can be approximated as it is, or if a transformation should be applied to increase the quality of the interpolation. Lastly, using the Expected Improvement Function, subsequent sample points are added to the initial set until a specified accuracy is reached.

As to the initial number of sample points, there is no formulae. Instead, as a rule of thumb, one should consider sampling a percentage of the design space equal to ten times the order of the design space [10]. 


\subsection{LATIN HYPERCUBE SAMPLING SCHEME}

When the exploration of a large design space is required, one often faces the problem of selecting the observation points. This choice of samples is not trivial, since local phenomena may be overlooked by either clustering of sample points around an area or by the lack of resolution of the grid size used to partition the design space [70]. To that end, the Latin Hypercube allows us to select the grid points of the design space at which the samples are to be measured or computed [71].

The Latin hypercube sampling is generalization (to an arbitrary number of dimensions) of the socalled Latin square, shown in Figure 3.1, in which, only one sample exists per each row and per column of a two-dimensional space. Therefore, in the Latin hypercube the sample points are chosen as to be the only point aligned in the hyperplane that contains it.

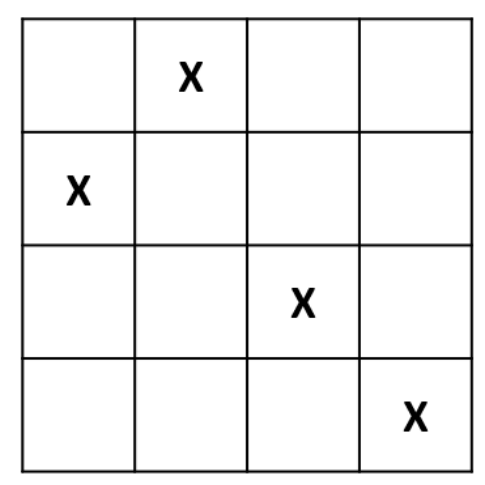

Figure 3.1. The Latin Square.

Let $\mathrm{n}$ be the number of samples and $\mathrm{p}$ the number of variables (or dimensions) of the design space. A $n \times p$ matrix $\mathbf{P}$ can be constructed, in which each of the $\mathrm{p}$ columns is a random permutation of the integers $0, \ldots, \mathrm{n}$; and a $n \times p$ matrix $\mathbf{R}$, in which a random distribution of numbers from 0 to 1 are assigned. Then, in its simplest form, the sampling space $\mathbf{S}$ is computed as: 


$$
\boldsymbol{S}=\frac{1}{n}(\boldsymbol{P}-\boldsymbol{R})
$$

\subsection{KRIGING INTERPOLATOR}

The Kriging can be regarded as a generalized linear regression model that seeks to minimize the mean square error between the estimated value and the true value by measuring the similarity (covariance) and dissimilarity (semi-variance) of the known neighbour sample points as a function of the distance between such points [56,72].

Consider that for a given set of known sample points $\boldsymbol{X}=\left\{\boldsymbol{x}_{1}, \boldsymbol{x}_{2}, \ldots, \boldsymbol{x}_{\boldsymbol{n}}\right\}$ there exists $n$ responses $\boldsymbol{Y}=\left\{\boldsymbol{y}\left(\boldsymbol{x}_{1}\right), \boldsymbol{y}\left(\boldsymbol{x}_{2}\right), \ldots, \boldsymbol{y}\left(\boldsymbol{x}_{\boldsymbol{n}}\right)\right\}$. The estimated response $\widehat{\boldsymbol{Y}}(\boldsymbol{X})$ can be described by the sum of a linear regression model and a component reflecting the influences of the independent variables $\boldsymbol{X}$ to the dependent variable $\boldsymbol{Y}$, namely $Z(\boldsymbol{X})$, which creates local deviations; this is the fundamental idea behind the Kriging predictor.

Considering that the set of known sample points can be described by a single variable, or by a set of variables, i.e. $\boldsymbol{x}_{\mathbf{1}}=\left(x_{1}, w_{1}\right)$, the Kriging predictor can be represented mathematically as:

$$
\widehat{\boldsymbol{Y}}(\boldsymbol{X})=f(\boldsymbol{X})^{T} \beta+Z(\boldsymbol{X})
$$

where $\beta$ are a set of unknown coefficients, $f(\boldsymbol{X})$ is a set of polynomial regression functions expressed by equation 3.3 and the superscript ()$^{T}$ is the linear algebraic vector transpose.

$$
f(\boldsymbol{X})=\left\{f\left(\boldsymbol{x}_{1}\right), f\left(\boldsymbol{x}_{2}\right), \ldots, f\left(\boldsymbol{x}_{\boldsymbol{n}}\right)\right\}
$$

The product $f(\boldsymbol{X})^{T} \beta$ is the global approximation of the response. Here, a second order polynomial of the following form is used: 


$$
f\left(\boldsymbol{x}_{\boldsymbol{i}}\right)^{T} \beta=f\left(x_{i}, w_{i}\right)^{T} \beta=\left[\begin{array}{llllll}
1 & x_{i} & w_{i} & x_{i}^{2} & x_{i} w_{i} & w_{i}^{2}
\end{array}\right]\left[\begin{array}{l}
\beta_{1} \\
\beta_{2} \\
\beta_{3} \\
\beta_{4} \\
\beta_{5} \\
\beta_{6}
\end{array}\right]
$$

This form of the Kriging is known as universal. When the set polynomial functions are equal to a set of ones (i.e. $f(\boldsymbol{X})=\{1\})$ the interpolator takes a form known as ordinary Kriging [51].

An analytical solution can be obtained for estimating the initial constant terms $\beta_{0}$ by means of the least square method as:

$$
\beta_{0}=\left(\boldsymbol{F}^{\boldsymbol{T}} \boldsymbol{F}\right)^{-1}\left(\boldsymbol{F}^{\boldsymbol{T}} \boldsymbol{Y}\right)
$$

Here, $\boldsymbol{F}$ is the matrix of polynomial functions $f(\boldsymbol{X})$ evaluated at the known sample points $\boldsymbol{X}=$ $\left\{\boldsymbol{x}_{1}, \boldsymbol{x}_{2}, \ldots, \boldsymbol{x}_{\boldsymbol{n}}\right\}$. The second term in equation 3.2, namely $Z(\boldsymbol{X})$, is a Gaussian stationary random process that possesses a zero-mean value and a covariance given by:

$$
\operatorname{Cov}[Z(\boldsymbol{X})]=\sigma^{2} \boldsymbol{R}\left(\boldsymbol{\theta}, \boldsymbol{x}_{\boldsymbol{i}}, \boldsymbol{x}_{\boldsymbol{j}}\right)
$$

Where $\sigma^{2}$ is the process variance and $\boldsymbol{R}\left(\boldsymbol{\theta}, \boldsymbol{x}_{\boldsymbol{i}}, \boldsymbol{x}_{\boldsymbol{j}}\right)$ is known as the Spatial Correlation Function (SCF), this is a function of the distance between sample points and an undefined parameter $\boldsymbol{\theta}$.

Furthermore, a correlation vector between the unknown points $\boldsymbol{X}$ and the known sample points is defined as $\boldsymbol{r}(\boldsymbol{X})$.

$$
\boldsymbol{r}(\boldsymbol{X})=\left\{\boldsymbol{R}\left(\boldsymbol{\theta}, \boldsymbol{x}, \boldsymbol{x}_{\boldsymbol{i}}\right), \boldsymbol{R}\left(\boldsymbol{\theta}, \boldsymbol{x}, \boldsymbol{x}_{i+1}\right), \ldots, \boldsymbol{R}\left(\boldsymbol{\theta}, \boldsymbol{x}, \boldsymbol{x}_{\boldsymbol{n}}\right)\right\}^{T}
$$

Now, let us assumes that a simple predictor, $\widehat{\boldsymbol{Y}}(\boldsymbol{X})$, can be built as a linear combination of unknown of coefficients $\zeta(\boldsymbol{X})^{T}$ and known $\boldsymbol{Y}$ responses. 


$$
\widehat{\boldsymbol{Y}}(\boldsymbol{X})=\zeta(\boldsymbol{X})^{T} \boldsymbol{Y}
$$

The error between the linear predictor and the true function $Y_{t}(\boldsymbol{X})$ is unknown. However, it can approximated by assuming that the true function can be represented by equation 3.2 [72]. Then, the error is given by:

$$
e=\widehat{\boldsymbol{Y}}(\boldsymbol{X})-Y_{t}(\boldsymbol{X})=\zeta(\boldsymbol{X})^{T}(\boldsymbol{F} \beta+Z(\boldsymbol{X}))-\left(f(\boldsymbol{X})^{T} \beta+z(\boldsymbol{X})\right)
$$

Keep in mind that $z(\boldsymbol{X})$ represents the deviations evaluated at the unknown points and $Z(\boldsymbol{X})$ represents the deviations evaluated at the known sample points. Rearranging equation 3.9 and factorizing the terms the following expression is found:

$$
e=\zeta(\boldsymbol{X})^{T} Z(\boldsymbol{X})-z(\boldsymbol{X})+\left(\zeta(\boldsymbol{X})^{T} \boldsymbol{F}-f(\boldsymbol{X})^{T}\right) \beta
$$

In the context of statistics, a predictor is said to be unbiased if the difference between the estimators expected value and the true value is zero [73]. To keep the predictor $\widehat{\boldsymbol{Y}}(\boldsymbol{X})$ unbiased, the second term of equation 3.10 becomes is set to zero, that is:

$$
\zeta(X)^{T} \boldsymbol{F}-f(\boldsymbol{X})^{T}=0
$$

Thus, the mean square error, $\phi(\boldsymbol{X})$ between the linear predictor and the true function can be estimated by taking the expected value of the unbiased square error:

$$
\phi(\boldsymbol{X})=E\left[e^{2}\right]=E\left[\left(\zeta(\boldsymbol{X})^{T} Z(\boldsymbol{X})-z(\boldsymbol{X})\right)^{2}\right]
$$

Similarly, to the least square method, the Kriging seeks to minimize the mean square error $\phi(X)$ as given by equation 3.12. In order to do so, $Z(\boldsymbol{X})$ and $z(\boldsymbol{X})$ are expressed in terms of the spatial correlation function chosen, which yields equation 3.13. 


$$
\phi(\boldsymbol{X})=\sigma^{2}\left(1+\zeta(\boldsymbol{X})^{T} \boldsymbol{R}\left(\boldsymbol{\theta}, \boldsymbol{x}, \boldsymbol{x}_{\boldsymbol{n}}\right) \zeta(\boldsymbol{X})-2 \zeta(\boldsymbol{X})^{T} \boldsymbol{r}(\boldsymbol{X})\right)
$$

The original problem has now been transformed into minimizing equation 3.13 with respect to the linear predictor $\zeta(X)^{T}$ subjected to the constraint equation 3.11. The solution is found using the Lagrange multiplier $(\lambda)$, which yields the following set of equations:

$$
\left[\begin{array}{cc}
\boldsymbol{R}\left(\boldsymbol{\theta}, \boldsymbol{x}, \boldsymbol{x}_{\boldsymbol{n}}\right) & \boldsymbol{F} \\
\boldsymbol{F}^{\boldsymbol{T}} & 0
\end{array}\right]\left[\begin{array}{c}
\zeta(\boldsymbol{X}) \\
\lambda
\end{array}\right]=\left[\begin{array}{c}
\boldsymbol{r}(\boldsymbol{X}) \\
f(\boldsymbol{X})
\end{array}\right]
$$

From equation 3.14, the solution of the minimization variables $\zeta(\boldsymbol{X})$ and $\lambda$ yields:

$$
\begin{gathered}
\lambda=\left(\boldsymbol{F}^{T} \boldsymbol{R}\left(\boldsymbol{\theta}, \boldsymbol{x}, \boldsymbol{x}_{\boldsymbol{n}}\right)^{-1} \boldsymbol{F}\right)^{-1}\left(\boldsymbol{F}^{T} \boldsymbol{R}\left(\boldsymbol{\theta}, \boldsymbol{x}, \boldsymbol{x}_{\boldsymbol{n}}\right)^{-1} \boldsymbol{r}(\boldsymbol{X})-f(\boldsymbol{X})\right) \\
\zeta(\boldsymbol{X})=\boldsymbol{R}\left(\boldsymbol{\theta}, \boldsymbol{x}, \boldsymbol{x}_{\boldsymbol{n}}\right)^{-1}(\boldsymbol{r}(\boldsymbol{X})-\boldsymbol{F} \lambda)
\end{gathered}
$$

Substituting equations 3.15 and 3.16 into 3.8 , the following relation is obtained :

$$
\begin{array}{rl}
\widehat{Y}(X)=r(X)^{T} & R\left(\theta, x, x_{n}\right)^{-1} Y \\
& -\left(F^{T} R\left(\theta, x, x_{n}\right)^{-1} r(X)\right. \\
& -f(X))^{T}\left(F^{T} R\left(\theta, x, x_{n}\right)^{-1} F\right)^{-1} F^{T} R\left(\theta, x, x_{n}\right)^{-1} Y
\end{array}
$$

Note that the term $\left(\boldsymbol{F}^{\boldsymbol{T}} \boldsymbol{R}\left(\boldsymbol{\theta}, \boldsymbol{x}, \boldsymbol{x}_{n}\right)^{-\mathbf{1}} \boldsymbol{F}\right)^{-1} \boldsymbol{F}^{\boldsymbol{T}} \boldsymbol{R}\left(\boldsymbol{\theta}, \boldsymbol{x}, \boldsymbol{x}_{n}\right)^{-\mathbf{1}} \boldsymbol{Y}$ has the form of equation 3.5. Indeed, equation 3.5 is the solution to the least square method when the correlation between the points is equal to the identity matrix, thus:

$$
\beta=\left(F^{T} R\left(\theta, x, x_{n}\right)^{-1} F\right)^{-1} F^{T} R\left(\theta, x, x_{n}\right)^{-1} Y
$$

Finally, substituting 3.18 into 3.17 and rearranging the terms, the predictor takes the form:

$$
\widehat{\boldsymbol{Y}}(\boldsymbol{X})=f(\boldsymbol{X})^{T} \beta+\boldsymbol{r}(\boldsymbol{X})^{T} \boldsymbol{R}\left(\boldsymbol{\theta}, \boldsymbol{x}, \boldsymbol{x}_{n}\right)^{-\mathbf{1}}(\boldsymbol{Y}-\boldsymbol{F} \beta)
$$


For a given set of sample points the parameters $\beta, \boldsymbol{R}\left(\boldsymbol{\theta}, \boldsymbol{x}, \boldsymbol{x}_{\boldsymbol{n}}\right)^{-\mathbf{1}}$ and the term in parenthesis $(\boldsymbol{Y}-\boldsymbol{F} \beta)$ are fixed. For every new point needed to calculate in the design space only the vectors $f(\boldsymbol{X})$ and $\boldsymbol{r}(\boldsymbol{X})^{\boldsymbol{T}}$ need to be computed.

The selection of the SCF $\left(\boldsymbol{R}\left(\boldsymbol{\theta}, \boldsymbol{x}, \boldsymbol{x}_{\boldsymbol{n}}\right)\right)$ will greatly modify the behaviour of the surrogate model, since it defines the level of influence between neighbouring points, i.e. it dictates the weight that the magnitude of a sample point has on the magnitude of a predicted point as a function of their distance. Take for example a continuous smooth function, such as a quadratic equation, in this scenario the correlation model should reflect that points far away from a sample are not or only slightly influenced by it, but points that lie in the close neighborhood are greatly influenced by it.

Certain restrictions exist as to which functions are suitable, commonly Gaussian exponential and linear functions are used. In this work, the Gaussian model is considered since it provides a smooth transition between levels of influence between neighboring points.

\subsubsection{The Gaussian Correlation Model}

The Gaussian function is expressed as equation 3.20 [72]. The parameter $\boldsymbol{\theta}$, as shown in Figure 3.2, controls the curvature of the function. Thus, it controls the level of influence that the sampled points have on the unknown points of the design space as a function of the distance $\left(\boldsymbol{x}_{\boldsymbol{j}}-\boldsymbol{x}_{\boldsymbol{i}}\right)$ between them. A value of 1 indicates that the points are perfectly correlated, whereas a value of zero indicates the correlation is non-existent.

$$
\boldsymbol{R}\left(\boldsymbol{\theta}, \boldsymbol{x}_{\boldsymbol{j}}, \boldsymbol{x}_{\boldsymbol{i}}\right)=e^{\left[-\boldsymbol{\theta}\left|\boldsymbol{x}_{\boldsymbol{j}}-\boldsymbol{x}_{\boldsymbol{i}}\right|^{2}\right]}
$$




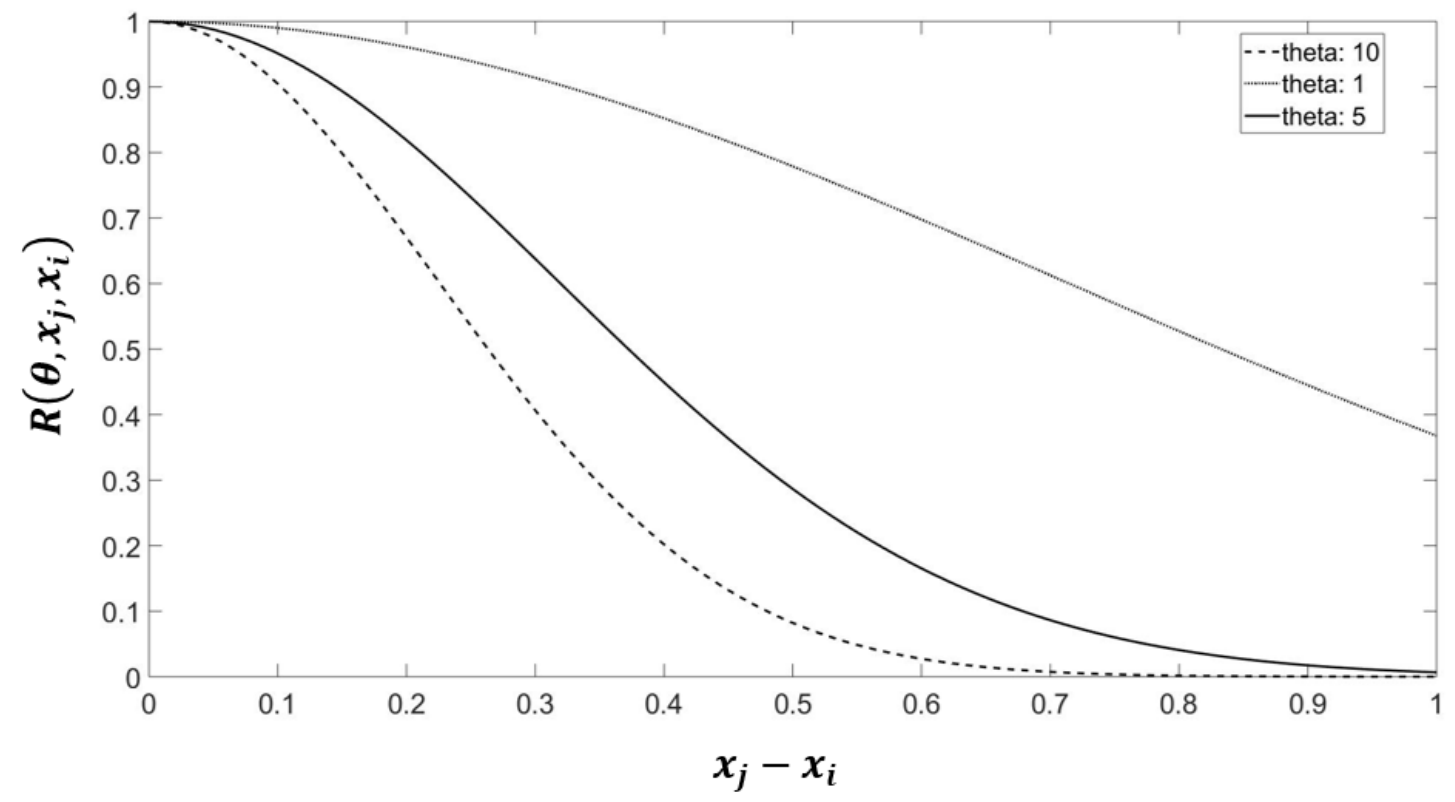

Figure 3.2. Gaussian covariance model.

To extend the analysis to multivariate, a univariate function is used for each of the dimension $p$ of the problem at hand. Using the product correlation rule [74], the special correlation function is given by:

$$
\boldsymbol{R}\left(\boldsymbol{\theta}, \boldsymbol{x}_{\boldsymbol{i}}, \boldsymbol{x}_{\boldsymbol{j}}\right)=\prod_{l=1}^{p} \boldsymbol{R}\left(\boldsymbol{\theta}_{l}\left|\boldsymbol{x}_{\boldsymbol{j}, l}-\boldsymbol{x}_{\boldsymbol{i}, l}\right|\right)
$$

The matrix of correlations $\boldsymbol{R}$ can then be assembled from all possible combinations of the known sample points $n$.

$$
R\left(\theta, x_{j}, x_{i}\right)=\left[\begin{array}{ccc}
R\left(x_{i}, x_{i}\right) & \cdots & R\left(x_{i}, x_{n}\right) \\
\vdots & \ddots & \vdots \\
R\left(x_{n}, x_{i}\right) & \cdots & R\left(x_{n}, x_{n}\right)
\end{array}\right]
$$

Note that the diagonal terms will all be equal to unity, since the correlation of a sample point with respect to itself is always one. Though by definition $\boldsymbol{R}\left(\boldsymbol{\theta}, \boldsymbol{x}_{\boldsymbol{j}}, \boldsymbol{x}_{\boldsymbol{i}}\right)$ should yield positive definite 
matrix, several issues arise due to round off errors and the distribution of the sample points selected. For example, a well-known difficulty arises when data points are too close to each other: the matrix becomes ill-conditioned. That is, the ratio between the highest and lowest eigenvalue is too large and the determinant of $\boldsymbol{R}\left(\boldsymbol{\theta}, \boldsymbol{x}_{\boldsymbol{j}}, \boldsymbol{x}_{\boldsymbol{i}}\right)$ is close to zero [75].

For practical applications, the eigenvalues of $\boldsymbol{R}\left(\boldsymbol{\theta}, \boldsymbol{x}_{\boldsymbol{j}}, \boldsymbol{x}_{\boldsymbol{i}}\right)$ are computed and if any negative eigenvalues are found the system is disturbed $m$ times until the system becomes positive definite. The disturbed correlation model is given by $R_{d}$ in the set of equations given in 3.23 , where $\mu$ is the disturbance magnitude, $I$ is the identity matrix and $\varepsilon_{r}$ is a constant related to the accuracy of the computation [72].

$$
\begin{gathered}
R_{d}=\boldsymbol{R}\left(\boldsymbol{\theta}, \boldsymbol{x}_{\boldsymbol{j}}, \boldsymbol{x}_{\boldsymbol{i}}\right)+\mu I \\
\mu=(10+m) \varepsilon_{r} \\
\varepsilon_{r}=2.22 \times 10^{-16}
\end{gathered}
$$

Though any choice of $\boldsymbol{\theta}$ would produce a surrogate model that interpolates exactly through the known data points, the goal is to find the parameters that has the highest probability of reproducing the behaviour of the correlated deviations $Z(\boldsymbol{X})[72,76]$. To that end, the Maximum Likelihood method is used, which is described briefly in the next subsection.

\subsubsection{The Maximum Likelihood Estimator}

The best Kriging parameters are found by maximizing the logarithm of the probability distribution function of the residuals, known otherwise as the Maximum Likelihood Estimator (MLE) [61]. 
It is assumed that the residuals possess a Gaussian probability distribution function, where the MLE takes the form of equation 3.24.

$$
\begin{aligned}
L\left[\boldsymbol{\theta}, \beta, \sigma^{2} \mid \boldsymbol{Y}\right]= & -\frac{n}{2} \ln (2 \pi)-\frac{n}{2} \ln \left(\sigma^{2}\right)-\frac{1}{2} \ln \left(\left|\boldsymbol{R}\left(\boldsymbol{\theta}, \boldsymbol{x}_{\boldsymbol{j}}, \boldsymbol{x}_{\boldsymbol{i}}\right)\right|\right) \\
& -\frac{1}{2 \sigma^{2}}(\boldsymbol{Y}-\boldsymbol{F} \beta)^{\boldsymbol{T}} \boldsymbol{R}\left(\boldsymbol{\theta}, \boldsymbol{x}_{\boldsymbol{j}}, \boldsymbol{x}_{\boldsymbol{i}}\right)^{-\mathbf{1}}(\boldsymbol{Y}-\boldsymbol{F} \beta)
\end{aligned}
$$

Where $\mathrm{n}$ is the total number of sample points. Since the negative logarithmic function is concave a first order optimality condition is enough to locate the best parameters $\boldsymbol{\theta}, \beta, \sigma^{2}$. Analytical solutions can be found for $\beta, \sigma^{2}$ and their optimal parameters are renamed $\hat{\beta}$ and $\hat{\sigma}^{2}$ which are expressed as:

$$
\begin{gathered}
\hat{\beta}=\left(\boldsymbol{F}^{\boldsymbol{T}} \boldsymbol{R}\left(\boldsymbol{\theta}, \boldsymbol{x}_{\boldsymbol{j}}, \boldsymbol{x}_{\boldsymbol{i}}\right)^{-\mathbf{1}} \boldsymbol{F}\right)^{-1} \boldsymbol{F}^{\boldsymbol{T}} \boldsymbol{R}\left(\boldsymbol{\theta}, \boldsymbol{x}_{\boldsymbol{j}}, \boldsymbol{x}_{\boldsymbol{i}}\right)^{-\mathbf{1}} \boldsymbol{Y} \\
\hat{\sigma}^{2}=\frac{1}{n}(\boldsymbol{Y}-\boldsymbol{F} \hat{\beta})^{\boldsymbol{T}} \boldsymbol{R}\left(\boldsymbol{\theta}, \boldsymbol{x}_{\boldsymbol{j}}, \boldsymbol{x}_{\boldsymbol{i}}\right)^{-\mathbf{1}}(\boldsymbol{Y}-\boldsymbol{F} \hat{\beta})
\end{gathered}
$$

There is no analytical solution for the parameter $\boldsymbol{\theta}$, instead equation 3.24 is re-written in terms of the known best parameters $\hat{\beta}, \hat{\sigma}^{2}$ and use a gradient based optimization method, as found in $[10,51,72]$, to solve for $\widehat{\boldsymbol{\theta}}$ :

$$
L[\boldsymbol{\theta} \mid \boldsymbol{Y}]=-\frac{n}{2} \ln (2 \pi)-\frac{n}{2} \ln \left(\hat{\sigma}^{2}\right)-\frac{1}{2} \ln \left(\left|\boldsymbol{R}\left(\boldsymbol{\theta}, \boldsymbol{x}_{\boldsymbol{j}}, \boldsymbol{x}_{\boldsymbol{i}}\right)\right|\right)-\frac{n}{2}
$$

As a consequence of profiling the parameters $\hat{\beta}, \hat{\sigma}^{2}$ into the MLE is that $\boldsymbol{\theta}$ tends to be biased. A solution to this problem is to use the restricted maximum likelihood functions (RMLE), which consists in calculating the log-likelihood of $n-p$ linearly independent contrasts, that is, linear combinations of observations whose joint distribution does not depend on the parameters $\hat{\beta}, \hat{\sigma}^{2}$. 
Here the mathematical development of the RMLE will not be addressed. However, the reader is directed to Zhang, X. [77] for further information.

\subsection{Model VAlidation ("CROSS-VAlidation")}

A simple approach to estimating the accuracy of the predictor, without sampling anymore points, relies on a "cross-validation" test. The basic idea is to remove one of the sampled data points and re-calculate the response surface $\widehat{\boldsymbol{Y}}_{\boldsymbol{i}-\mathbf{1}}(\boldsymbol{X})$ and its corresponding mean square error $\phi_{-i}(\boldsymbol{X})$ using the remaining $n-1$ observations.

The number of standard errors between the estimated surface, $\hat{Y}_{-\boldsymbol{i}}\left(\boldsymbol{x}_{\boldsymbol{i}}\right)$, and the known value, $\boldsymbol{Y}\left(\boldsymbol{x}_{\boldsymbol{i}}\right)$, can be calculated using the Standardized Cross-Validated Residual (SCVR) as [10]:

$$
S C V R=\frac{\boldsymbol{Y}\left(\boldsymbol{x}_{\boldsymbol{i}}\right)-\hat{Y}_{-\boldsymbol{i}}\left(\boldsymbol{x}_{\boldsymbol{i}}\right)}{\sqrt{\phi_{-\boldsymbol{i}}\left(\boldsymbol{x}_{\boldsymbol{i}}\right)}}
$$

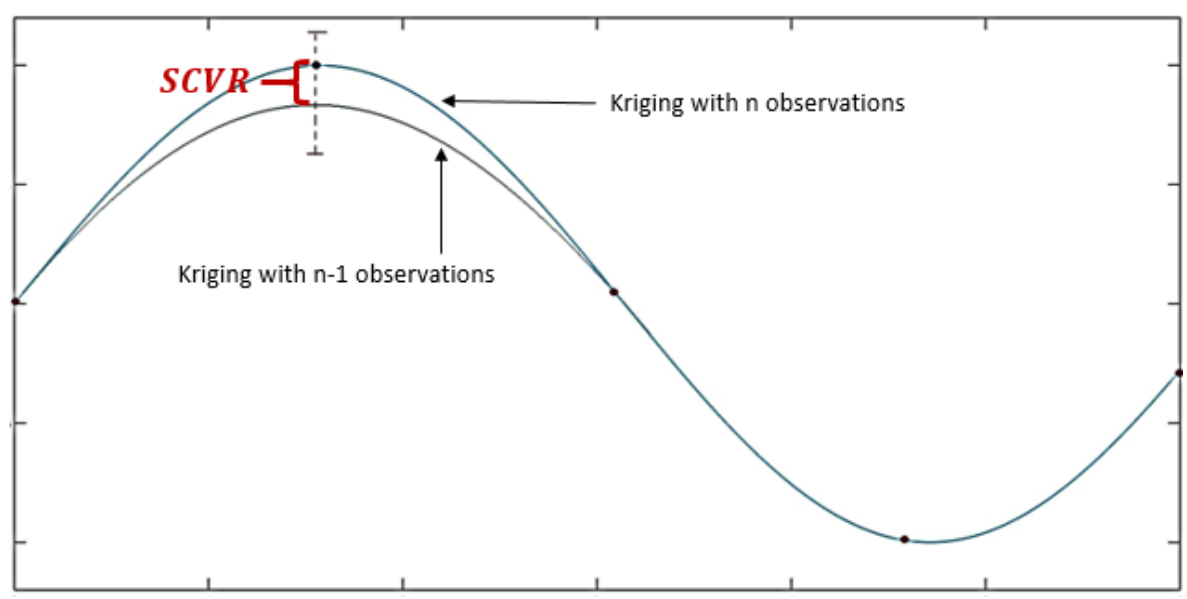

Figure 3.3. Standardized Cross-Validated Residual. One sample point is removed from the sample vector and the surface response is re-interpolated using the Kriging. 
The Standardized Cross-Validated Residual $(S C V R)$, as given per equation 3.28, is simply a confidence interval as can be seen in Figure 3.3 [10]. The model is said to be valid, if the SCVRS lie within a $[-3,+3]$ interval. When a model fails the diagnostic, it is recommended that a transformation be performed, of the kind $\ln (\boldsymbol{Y})$, to improve the quality of the fit, or to increase the initial number of sample points selected for the fit .

\subsection{THE EXPECTED IMPROVEMENT FUNCTION}

The Expected Improvement Function is one of several "infill criterion" used to select subsequent observations after an initial fit [10]. The main idea here is to model the uncertainty about the function's value at a point as the realization of a normally distributed random variable with mean, standard deviation and root mean square error as given by the Kriging predictor. This method provides a good trade-off between choosing saddle points and points with high uncertainty [78].

Let $f_{\min }=\min \left(\boldsymbol{Y}\left(\boldsymbol{x}_{\boldsymbol{i}}\right), \ldots, \boldsymbol{Y}\left(\boldsymbol{x}_{\boldsymbol{n}}\right)\right)$, be the current minimum function value. If a new sample point is to be chosen, this point should improve our current estimate of $f_{\min }$, thus the improvement function $I(\boldsymbol{X})$ is written as:

$$
I(\boldsymbol{X})=\max \left(f_{\min }-\boldsymbol{Y}(\boldsymbol{X}), 0\right)
$$

To obtain the Expected Improvement, the expected value of equation 3.29 is taken.

$$
E[I(\boldsymbol{X})]=E\left[\max \left(f_{\min }-\boldsymbol{Y}(\boldsymbol{X}), 0\right)\right]
$$

The closed from solution of equation 3.30 takes the form:

$$
E[I(\boldsymbol{X})]=\left(f_{\min }-\widehat{\boldsymbol{Y}}(\boldsymbol{X})\right) \Psi\left(\frac{f_{\min }-\widehat{\boldsymbol{Y}}(\boldsymbol{X})}{\sqrt{\phi(\boldsymbol{X})}}\right)+\psi\left(\frac{f_{\min }-\widehat{\boldsymbol{Y}}(\boldsymbol{X})}{\sqrt{\phi(\boldsymbol{X})}}\right) \sqrt{\phi(\boldsymbol{X})}
$$


Where $\Psi(\cdot)$ is the standard normal distribution function and $\psi(\cdot)$ is the standard normal density. The new infill point is then selected as the point where the Expected Improvement is maximum:

$$
\max (E[I(\boldsymbol{X})])
$$




\section{THEORETICAL FRAMEWORK PART III: MODAL CONTRIBUTION FACTORS}

This chapter presents the second main block that construct the surrogate-aided loads algorithm. It intents to exploit the idea of measuring, in a computationally cheap manner, how significant the change in the monitored loads is after structural modification during design optimization.

To begin, let's recall that the complex behaviour of a dynamic structure can be decomposed into a set of simple harmonic functions linearly coupled with amplitude ratios. This is achieved by finding the eigenvalues and eigenvectors that satisfy the characteristic equation, namely, the undamped free vibration problem as given in equation 2.9. In the real world, all systems are continuous and they all possess an infinite number of degrees of freedom and thus an infinite number of eigenvectors, however only a fraction of those modes are necessary to approximate the response.

Hence, if the modes that will dominate the response could be identified and their contribution assessed, some information could be draw about the response itself.

The concept of modal contribution factor, however, seems to be ambiguous. A simple search of the words "modal participation factor" will quickly reveal that several variants of this concept exist and that it is used interchangeably with the term "modal contribution factors". For example, in electrical engineering [79-82] the term refers to the product between the left and the right eigenvectors. On the other hand, MacNeal, R., Irvine, T. and Girard, A., \& Roy, N.A. [83-85] refer to the modal mass participation factor as simply modal participation factor, while Kuhar, E.J. \& Stahle, C.V. [86] uses the same term to refer to the components of the eigenvector matrix. Chen, 
J.T., et al., Chen, J.T., Hong, H.K., \& Yeh, C.S., Wison, E.L., Yuan, M.W., \& Dickens, J.M., Carlbom, P.F., Salmonte, A.J., Photiadis, D.M. et al., and Wallrapp, O., \& Wiedemann, S. [8793] each define a convenient mathematical form defined as modal participation factor.

In the following subsection, a unified concept is presented, along with a simple classification of the different definitions available in the literature relevant to structural engineering.

A simple 5 degree of freedom model is used as proof of concept to determine which metric would be preferable to use in the surrogate-aided algorithm. However, for convenience and for the sake of being concise, the comparative results of this chapter are presented in detail in the following chapter.

\subsection{Modal CONTRIBUtion FACTORS}

The complex response of a dynamic structural system can be deconstructed into a set of simple harmonic functions, $q_{i}(t)$, also known as modal responses, linearly coupled with amplitude ratios. This transformation is achieved by finding the system modal pairs, in the form of natural frequencies, $\omega_{n_{i}}$, and mode shapes, $\left\{\varphi_{i}\right\}$, that satisfy its characteristic equation. The equation of motion for an undamped free vibration system with $m$ modes retained in the solution:

$$
\{u(t)\}=\sum_{i=1}^{m}\left\{\varphi_{i}\right\} q_{i}(t)=\varphi_{j, 1} q_{1}(t)+\varphi_{j, 2} q_{2}(t)+\cdots+\varphi_{j, m} q_{m}(t)
$$

where the subscript $j$ indicates the grid point number. From equation 4.1 the modal contribution can be defined as the quantity of movement that each mode grants to the total nodal response [9495]. In other words, it is the product between the modal response $q_{i}(t)$ and the amplitude ratio $\varphi_{j, i}$, denoted here with the Greek letter gamma $(v)$ as: 


$$
v_{j, i}=\varphi_{j, i} q_{i}(t)
$$

We will emphasize the distinction between modal contribution and a second family of parameters referred to as modal participation factors. The former indicates the kinematic contribution of the $i$-th mode to the $j$-th nodal response, as given by equation 4.2 . The latter aims to rank the relative importance of the mode by either approximating the value of $q_{i}(t)$, or by drawing assumptions about the behaviour of the system based on the eigenvector matrix, $\Phi$.

\subsubsection{Class I: Static Modal Participation Factors (SMPF)}

The amplitude of each modal response can be expressed as the product of a constant, $\Gamma_{i}$, and a time dependent term, $D_{i}(t)$, [94-96] which is expressed as:

$$
q_{i}(t)=\Gamma_{i} D_{i}(t)
$$

Though in general the time history of the response may be of interest, it is the value of the maximum displacement that will be used for the sizing or the design optimization of a structure. Thus, from equation 2.8 , the maximum value of the response in the physical domain is directly proportional to the maximum amplitude of the time dependent term, which is expressed as:

$$
\{u\}^{0}=\Phi[\Gamma]\{D\}^{0}
$$

where $\{u\}^{0}$ is the vector of maximum nodal displacements, $\{D\}^{0}$ is the dynamic modal response vector subjected to a unitary force, and $[\Gamma]$ is the diagonal matrix of modal participation factors. To find appropriate expressions for $[\Gamma]$ and $\{D\}^{0}$ a static modal decomposition will be performed.

First, the vector of external forces, $F(t)$, is decomposed into two components: a time invariant vector $f$ and a time dependent expression $p(t)$ as: 


$$
F(t)=f p(t)
$$

Since the vector $f$ is independent of time, it can be expanded into a matrix of static forces $f^{s t}$ acting at each grid point $j$ and within each mode $i$; where the sum of the columns of $f^{s t}$ is equal to the magnitude of the $j$-th component of the vector $f$ [94-97] which is expressed as:

$$
f_{j, i}^{s t}=\Gamma_{i, i} \varphi_{j, i}\left\{\begin{array}{l}
\text { for } i=1,2, \ldots, m \\
\text { for } j=1,2, \ldots, N
\end{array}\right.
$$

where $N$ and $m$ are the number of grid points and number of modes retained in the solution, respectively, and:

$$
\Gamma=\operatorname{diag}\left(\Phi^{T} f\right)
$$

When the eigenvectors are normalized with respect to mass, the vector of total static displacement can be found by the use of Hooke's law, which yields:

$$
u_{j}^{s t}=\sum_{i=1}^{m} \frac{\Gamma_{i, i}}{\omega_{n_{i}}^{2}} \varphi_{j, i}
$$

The vector of total static displacement can then be related to the vector of total dynamic displacements by substituting equation 4.8 into 4.4 , which results in:

$$
u_{j}^{s t}=u_{j}^{s t} \sum_{i=1}^{m} \omega_{n_{i}}^{2} D_{i}^{0}
$$

To derive an appropriate expression for $D^{0}$, lets recall the classical equation of motion in the modal coordinates, where the eigenvectors have been mass-normalized:

$$
\ddot{q}(t)+\omega_{n}^{2} q(t)=\Phi^{T} f p(t)
$$


Where the operator (*) denotes the second derivative with respect to time.

By introducing equations 4.3 and 4.7 into 4.10 , equation 4.11 is obtained, where the term $\Gamma$ is factored out of the expression, resulting in:

$$
\ddot{D}(t)+\omega_{n}^{2} D(t)=p(t)
$$

Equation 4.11 is simply the harmonic functions subjected to a unit dynamic load. Thus, depending on the form of the excitation force $p(t)$, a particular method can be selected to find an analytical solution to 4.11 and consequently for $D^{0}$.

\subsubsection{Steady-state Response}

When $p(t)$ is a periodic function, a dynamic amplification factor $R_{i}$ can be defined as the ratio between the amplitude of the dynamic response $D_{i}^{0}$ and the static response $D_{i}^{s t}[94]$ expressed as:

$$
R_{i}=\frac{D_{i}^{0}}{D_{i}^{s t}}
$$

The amplitude of the static response is found by neglecting the contribution of the acceleration term in the mass-normalized modal equations of motion, yielding equation 4.13.

$$
D_{i}^{s t}=\frac{1}{\omega_{n_{i}}^{2}}
$$

While the dynamic amplification factor is the steady-state amplitude of an undamped system subjected to a unit periodic function, expressed here as equation 4.14 .

$$
R_{i}=\frac{1}{\omega_{n_{i}}^{2}-\omega^{2}}
$$


By introducing equations 4.12 through 4.14 , into 4.9 , an inexpensive expression which allows estimating the maximum amplitude of the physical displacements during steady-state vibration is found, which is expressed as:

$$
\{u\}^{0}=\{u\}^{s t}\{R\}
$$

To compare the output of this method with respect to the proposed methodology, a steady-state modal participation fraction $L_{i}^{s t}$ is defined as equation 4.16.

$$
L_{i}^{s t}=\frac{\left|\Gamma_{i} D_{i}^{o}\right|}{\sum_{i=1}^{m}\left|\Gamma_{i} D_{i}^{o}\right|}=\frac{\left|\Gamma_{i} /\left[\omega_{n_{i}}^{2}\left(\omega_{n_{i}}^{2}-\omega^{2}\right)\right]\right|}{\sum_{i=1}^{m}|| \Gamma_{i} /\left[\omega_{n_{i}}^{2}\left(\omega_{n_{i}}^{2}-\omega^{2}\right)\right]||}
$$

This fractional number allows the comparison of the relative amplitudes of the modal responses $q_{i}(t)$ for a given design and loading condition.

The right-hand side of equation 4.16 computes the normalized magnitude of the steady-state modal response, highlighted with black dots in Figure 4.1. There, it becomes clearer that the transient portion of the motion is neglected.

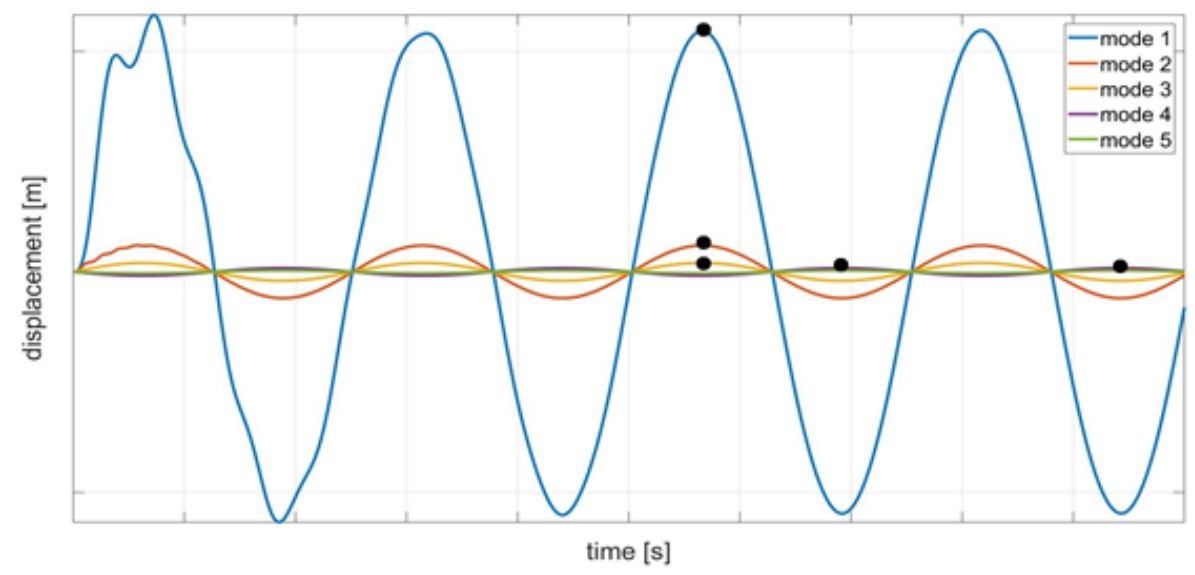

Figure 4.1. Time history of a dynamic system. The black dots indicate the points computed by the steady-state response participation factor. 


\subsubsection{Transient Response I}

The steady state participation factors neglect the contribution of the transient components of the time domain solution. When the global maximum is of interest, the goal is to estimate the maximum dynamic amplitude of system responses, namely:

$$
D_{i}^{t s}=\max \left(D_{i}(t)\right)
$$

Equation 4.17, locates the maximum of each modal response, as depicted in Figure 4.2. Notice, that the points estimated occur at different point in time.

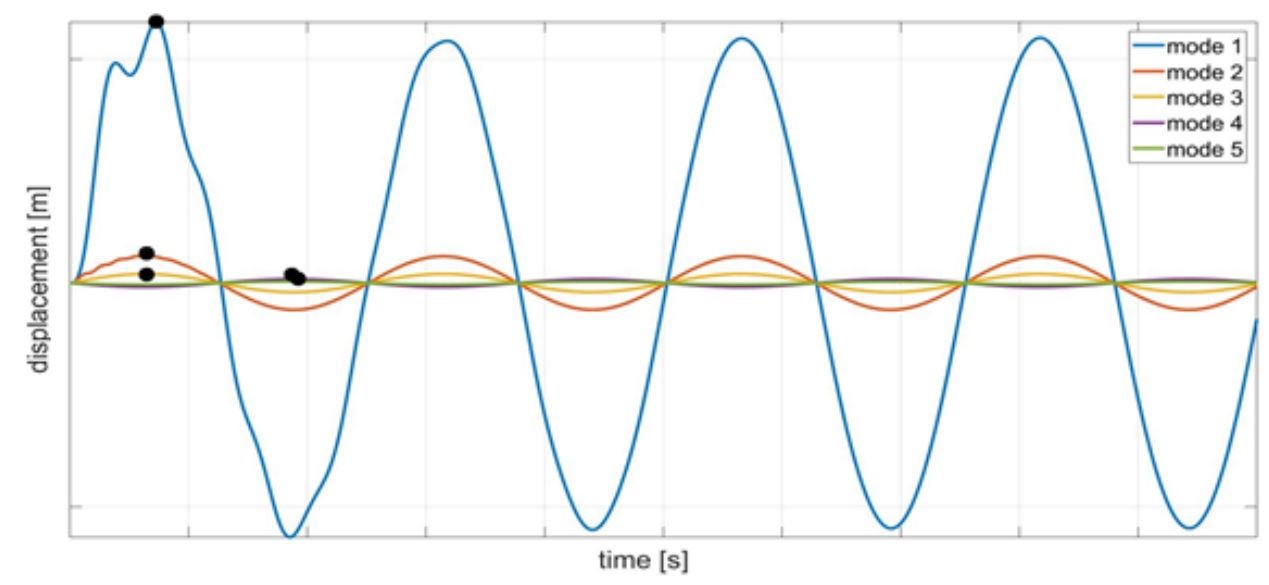

Figure 4.2. Time history of a dynamic system. The black dots indicate the points computed by the transient response I participation factor.

Then, a transient participation fraction $L_{i}^{t s}$ can be defined similarly to the steady participation fraction as the ratio between the maximum amplitude of the harmonic solution and the sum of the maximum amplitude of all the modes retained in the system, expressed mathematically as:

$$
L_{i}^{t s}=\frac{\left|\Gamma_{i} D_{i}^{t s}\right|}{\sum_{i=1}^{m}\left|\Gamma_{i} D_{i}^{t s}\right|}
$$




\subsubsection{Transient Response II - Analytical solutions}

To obtain equation 4.17 , the solution of the modal equations of motion is required a priori. As shown in [93] the solution of equation 4.17 can be approximated by using a quasi-static solution. Here, a new participation factor based on the closed form solution of a Single Degree of Freedom System (SDoF) is introduced.

Since this method requires the analytical solution of the modal equations of motion in the time domain, an excitation in the form of equation 4.19 is used as an example.

$$
p(t)=1-\cos (\omega t)
$$

This excitation is representative of the analytical model of the Tuned Discreet Gust (TDG) of an aero structure and thus relevant to the current study. However, it should be emphasized that the methodology is applicable for any forcing function where a close form solution exists.

The analytical solution in the time domain for an undamped SDoF system subjected to a unitary pulse such as 4.19 is given by:

$$
D_{i}=\left\{\begin{array}{cc}
\frac{1}{\omega_{n_{i}}^{2}}\left[1-\cos \left(\omega_{n_{i}} t\right)\right]-\frac{2}{\omega^{2}-\omega_{n_{i}}^{2}} \sin \left[\frac{\left(\omega_{n_{i}}+\omega\right) t}{2}\right] \sin \left[\frac{\left(\omega_{n_{i}}-\omega\right) t}{2}\right] & 0 \leq t \leq \tau \\
\frac{1}{\omega_{n_{i}}^{2}}\left[\cos \left(\omega_{n_{i}}(t-\tau)\right)-\cos \left(\omega_{n_{i}} t\right)\right]+\frac{1}{2 \omega_{n_{i}}\left(\omega-\omega_{n_{i}}\right)}\left[\cos \left(\left(\omega-\omega_{n_{i}}\right) \tau+\omega_{n_{i}} t\right)-\cos \left(\omega_{n_{i}} t\right)\right]-\cdots & \tau \leq t \\
\frac{1}{2 \omega_{n_{i}}\left(\omega+\omega_{n_{i}}\right)}\left[\cos \left(\left(\omega+\omega_{n_{i}}\right) \tau+\omega_{n_{i}} t\right)-\cos \left(\omega_{n_{i}} t\right)\right] &
\end{array}\right.
$$

where $\tau$ is the pulse length. From classical theory of shock, the maximum response will occur during the application of the pulse whenever the forcing frequency is smaller than twice the natural frequency of the system [98]. Thus, the solution for the maximum amplitude of the dominant elastic mode and the time $t_{d o m}^{\max }$ at which it occurs can be estimated by differentiating equation 4.20 with respect to time during the application of the pulse and finding the roots of the derivative. 
Alternatively, computing the complete time response of the dominant mode using 4.20 and extracting the peak values.

Then, the rest of the dynamic responses can be estimated by computing 4.20 at $t_{d o m}^{\max }$, as shown in Figure 4.3 and expressed mathematically as:

$$
D_{i}^{t s I I}=\max \left(D_{i}\left(t_{d o m}^{\max }\right)\right)
$$

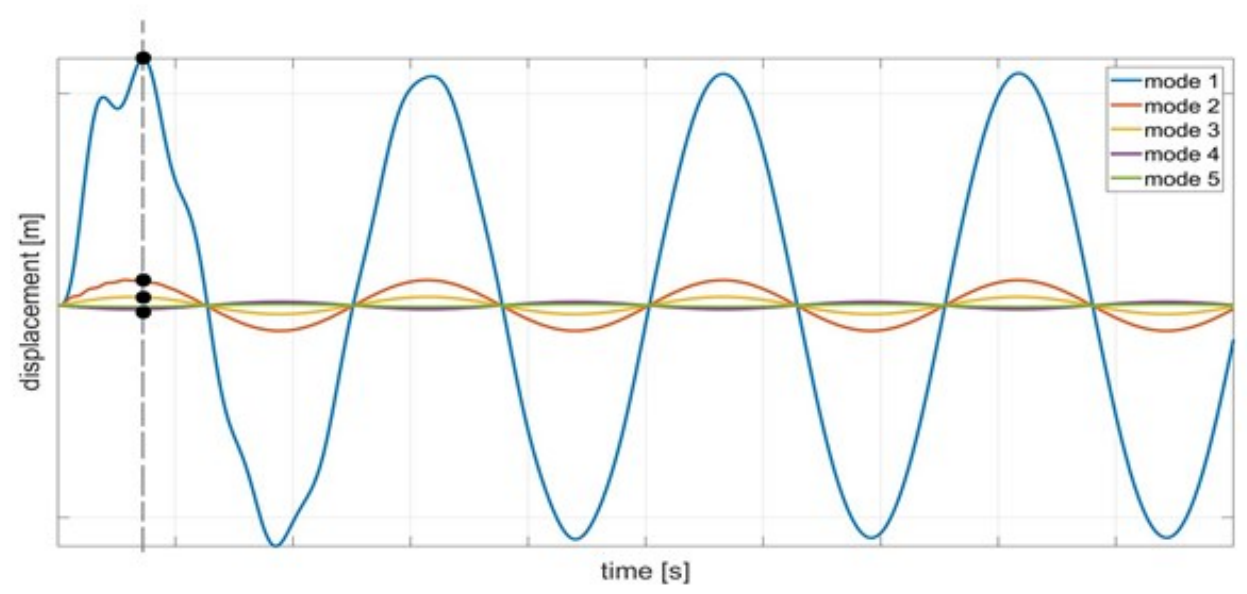

Figure 4.3. Time history of a dynamic system. The black dots indicate the points computed by the transient response II participation factor.

The transient response II participation fraction is defined similar to equations 4.16 and 4.18 as:

$$
L_{i}^{t s I I}=\frac{\left|\Gamma_{i} D_{i}^{t s I I}\right|}{\sum_{i=1}^{m}\left|\Gamma_{i} D_{i}^{t s I I}\right|}
$$

\subsubsection{Internal load Participation Factor}

For point loads, the element internal forces are computed as the product of the element stiffness matrix $\left[K_{e}\right]$ and the corresponding displacement vector $\left\{u_{e}(t)\right\}$ of the grids that conform to the element in the global coordinate system. 
When the element and global coordinate systems do not coincide a rotation matrix $[C]^{T}$ is necessary to ensure that the internal forces computed, $\left\{N_{e}(t)\right\}$, are expressed in the element coordinate system as:

$$
\left\{N_{e}(t)\right\}=[C]^{T}\left[K_{e}\right]\left\{u_{e}(t)\right\}
$$

Equation 4.23 can be expanded into its modal components as:

$$
\left\{N_{e}(t)\right\}=[C]^{T}\left[K_{e}\right]\left\{u_{e}(t)\right\}=[C]^{T}\left[K_{e}\right]\left[\varphi_{e}\right][\Gamma]\{D(t)\}
$$

Equation 4.24 gives an important insight into the variables that affect the internal loads sustained by each element. The term $[\Gamma]$ is constant for a given loading conditions and its magnitude depends on the matrix of eigenvectors. On the other hand, the magnitude of $\{D(t)\}$ is strongly dependent on the initial conditions and the ratio between the forcing and natural frequencies of the system. The magnitude of $[\Gamma]\{D(t)\}$, thus, determines the dominance of a mode in the solution, whereas the product $\left[\Sigma_{e}\right]=[C]^{T}\left[K_{e}\right]\left[\varphi_{e}\right]$ determines the impact of such mode in the $r$ component of the element load where $r \in\{1: 6\}$. A participation factor can then be defined as:

$$
L_{r, i}^{\text {load }}=\frac{\left|\sum_{e_{r, i} \Gamma_{i} D_{i}^{0}}\right|}{\sum_{i=1}^{m}\left|\sum_{e_{r, i}} \Gamma_{i} D_{i}^{0}\right|}
$$

\subsubsection{Class II: Relative Importance of a Mode}

This second class of modal participation factors aims to identify the harmonic functions that are easily excited. Many of these definitions try to exploit the structure of the modal equations of motion, to find a constant parameter, that could draw information about the systems behaviour without solving any differential equation of motion. 
The most widely known definition, that falls in this category, is the modal effective mass participation factor. It is used to determine the number of modes that should be retained to recover the solution in the physical domain $[84,99]$. However other definitions have been proposed in the literature. As an example, Wilson [89] defined a mode participation factor similar to equation 4.7 to determine which modes should be retained in subsequent analysis. In the context of rail vehicles, Carlbom et al. [90] introduced a criterion, based on four parameters, to identify dominant modes. Though many more definitions exist, here, the focus is restricted to definitions relevant to structural analysis.

\subsubsection{Modal Effective Mass Participation Factor}

The modal effective mass was initially derived for a SDoF system subjected to forced acceleration through the base. Its derivation proves that the effective mass of the system is directly proportional to the magnitude of the inertial force $F$. Thus, a large modal effective mass indicates a large inertial force, due to the enforced acceleration $\ddot{U}$.

The extension of this concept to multi-degree of freedom systems (MDoF), is attained by performing a Craig-Brampton transformation [99], which yields equation 4.26.

$$
F=M^{e f f}\left[1+\left(\frac{\omega}{\omega_{n}}\right)^{2} H\left(\frac{\omega}{\omega_{n}}\right)\right] \ddot{U}
$$

Where, $H\left(\frac{\omega}{\omega_{n}}\right)$ represents the system's transfer function and $M^{e f f}$ is the so-called modal effective mass, defined mathematically as:

$$
M_{p, i}^{e f f}=\frac{\Gamma_{p, i}^{m m T} \Gamma_{i, p}^{m m}}{M_{q_{i}}}
$$


This parameter indicates the effective mass of an elastic mode associated with a rigid-body mode. In fact, the summation of the masses associated with each elastic mode $i$ per rigid-body mode $p$ is equal to the mass of the rigid-body degree of freedom $p$.

The constant $M_{q_{i}}$ is the modal mass of the i-th eigenvector in equation 4.27 and $\Gamma^{m m}$ is the modal effective mass participation factor. The later relates the rigid-body modes $\varphi^{r}$ of size $n \times p$ (grid points x rigid-modes), with the elastic modes $\varphi^{e}$ of size $n \times m$ (grid points $\mathrm{x}$ elastic-modes) by:

$$
\Gamma^{m m^{T}}=\varphi^{r T} M \varphi^{e}
$$

Though the definition of the modal effective mass is derived assuming a free-free MDoF system, an important point should be kept in mind: the modal characteristics of the elastic degrees of freedom must be computed with respect to a selected degree of freedom, called boundary DoF, which should be constrained for the analysis. In fact, if this remark is not considered, the results extracted using equation 4.27 are ill-founded as the participation of the elastic DoF's will tend to zero for free-free systems.

This key remark is tightly wounded to the Craig-Brampton transformation. For further details, the reader is referred to De Silva, C.W. and Wijker, J.J. [98-99]. Finally, for comparison purposes an effective modal mass fraction can be defined as the ratio between the effective modal mass and the total mass of the system:

$$
L_{p, i}^{m m}=\frac{M_{p, i}^{e f f}}{\sum_{j=1}^{N} m_{j}}
$$




\subsubsection{Free-Free Modal Participation Factors}

Based on the concept of Effective Interface Mass (EIM), Kammer et al. [100] recently introduced a new definition to assess the relative importance of each mode for free-free systems.

By introducing the variable change, as per equation 2.8 , into the classical equation of motion of an undamped SDoF system and solving for the physical acceleration one obtains:

$$
\ddot{u}(t)=\Phi \ddot{q}(t)=\Phi \Phi^{T} f p(t)-\Phi \omega_{n_{i}}^{2} q(t)
$$

In the context of structural dynamics, the product $\Phi \Phi^{T}$ is classically regarded as the relationship between the response of the $j$-th grid point when the $n$-th grid is excited. From 4.30, this interpretation can be expanded by considering the product $\Phi \Phi^{T} f$, such that, the modes that are strongly excited by the external force vector are, in consequence, expected to contribute strongly to the physical response.

To assess such contribution, as defined in [100], the trace of the product between two eigenvectors is taken. Then, the free-free modal participation factor takes the form of equation 4.31.

$$
\Gamma_{i}^{f r e e}=\operatorname{tr}\left(\varphi_{i} \varphi_{i}^{T}\right)
$$

To compare the output of 4.31 , to the rest of the definitions presented here, a free-free modal participation fraction is defined as the ratio between the free-free participation factors and the trace of the elastic-degrees of freedom $\Gamma^{\text {elas }}$. That is:

$$
L_{i}^{\text {free }}=\frac{\Gamma_{i}^{\text {free }}}{\Gamma^{e l a s}}=\frac{\operatorname{tr}\left(\varphi_{i} \varphi_{i}{ }^{T}\right)}{\operatorname{tr}\left(\varphi^{e} \varphi^{e^{T}}\right)}
$$




\subsubsection{Modal Strain Energy Participation Factor}

The very well-known expression for strain energy $S E(t)$ is given here by equation 4.33 .

$$
S E(t)=\frac{1}{2} u(t)^{T} K u(t)
$$

The strain energy time history can be decomposed into its modal components, where the familiar term $K_{q}$ appears. If the eigenvectors were mass normalized, then the matrix of modal stiffness is equivalent to the diagonal matrix of eigenvalues. If our interest lies in the peak value, a matrix of peak strain energies, $S E^{\max }$, of size $m x N$ can be found

$$
S E^{\max }=\frac{1}{2} q^{\max T} \Phi^{T} K \Phi q^{\max }=\frac{1}{2} q^{\max T} K_{q} q^{\max }
$$

The strain energy matrix, as presented in equation 4.34 can be expensive to evaluate, since the solution of the maximum magnitude of the harmonic modal responses is necessary. Instead several authors [101-103] have proposed the use of the element modal strain energy $M S E_{j i}$ as assessment criteria. The $M S E_{j i}$ evaluates the participation of each mode at each element stiffness submatrix $K_{e}$ in the global coordinate system that composes the global stiffness matrix $K$.

$$
M S E_{j i}=\frac{1}{2} \varphi_{j, i}^{T} K_{e} \varphi_{j, i}
$$

A modal strain participation fraction $L_{e i}^{M S E}$, can be defined, as in [101], as the ratio between the strain energy and the kinetic energy of the system in free vibration. Thus:

$$
L_{e i}^{M S E}=\frac{\varphi_{j, i}^{T} K_{e} \varphi_{j, i}}{\Phi_{i}^{T} M \Phi_{i} \omega_{n_{i}}^{2}}
$$


A large modal strain energy fraction at the element $j$-th in the mode $i$-th indicates that such an element is a major load carrying component for such a mode. Thus, any structural modifications in that element will produce significant changes in the behaviour of its dominant mode.

When several modes are of interest, a coefficient can be constructed as the average of the summation of the modal strain energies of all the modes considered [102,103].

$$
M S E C_{j}=\frac{1}{m} \sum_{i=1}^{m} M S E_{j i}
$$

\subsection{PROOF OF CONCEPT}

A simple five degree of freedom model was created to assess the output of each of the metrics described previously in this chapter. Through this comparison, a suitable metric may be found, that allows to draw information about the system's behaviour, without numerically solving the equations of motion. Let us begin by describing the model characteristics.

The model consists of a cantilever beam with five beam elements, as shown in Figure 4.4 . Where each beam element has a stiffness of $8700[\mathrm{~N} / \mathrm{m}]$ and the model is undamped. It is excited by a 1 minus cosine shock (as given by equation 4.19), with a magnitude of $100 \mathrm{~N}$ acting at the fifth grid point and a forcing frequency of $22.4 \mathrm{rad} / \mathrm{sec}$. i.e., the pulse duration is 0.28 seconds. 


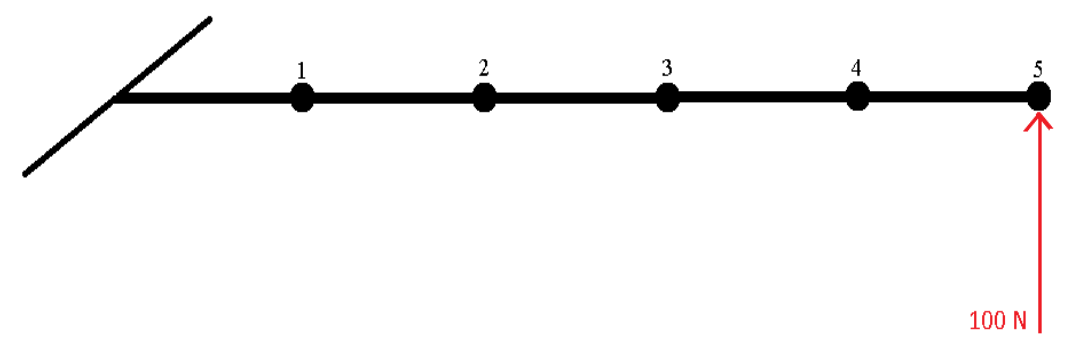

Figure 4.4. Proof of concept: the cantilever beam model.

To give the reader a better insight, the modal equations of motion were first solved in the time domain using a fourth order numerical method Runge-Kutta's, and the result is presented in Figure 4.5. Where the time domain histories of the modal response and the physical displacements of the five elements are presented as A) and B) respectively. The time history of the element in-plane shear force was also estimated and is presented as Figure 4.6. Comparing Figure 4.5 A) with Figure 4.5 B) and Figure 4.6, it seems clear that the physical displacements follow closely the behaviour of the first mode. The internal load also follows the behaviour of the first mode, except there is a slight phase angle between the time histories during the pulse application period.

Now, let's compute the modal steady-state $L_{i}^{s t}$, transient $L_{i}^{t s}$, transient- analytical solution $L_{i}^{t s I}$, modal effective mass $L_{i}^{m m}$, and free-free $L_{i}^{f f}$ participation fractions using equations $4.16,4.18$, $4.22,4.29$, and 4.32 respectively. The results are presented in the following subsection. 
(A)

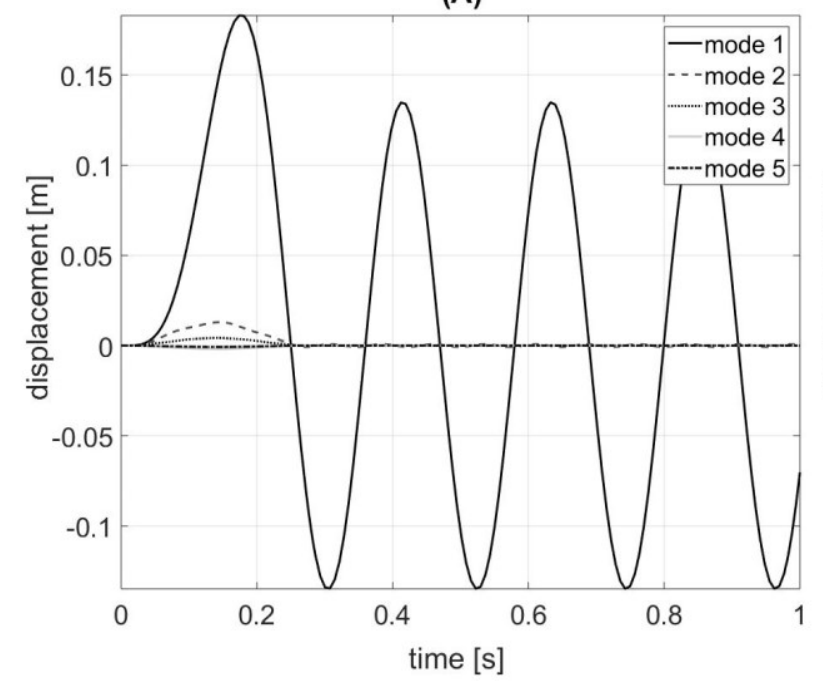

(B)

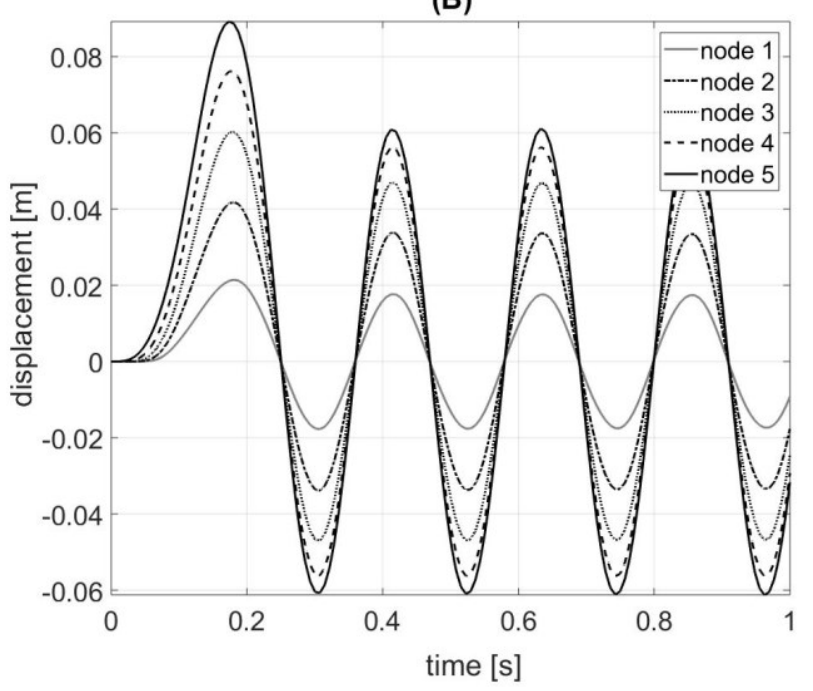

Figure 4.5. Solution of the modal equations of motion. A) Presents the behavior of the generalized coordinates. B) Presents the physical displacement of the grid points numbered according to Figure 4.4.

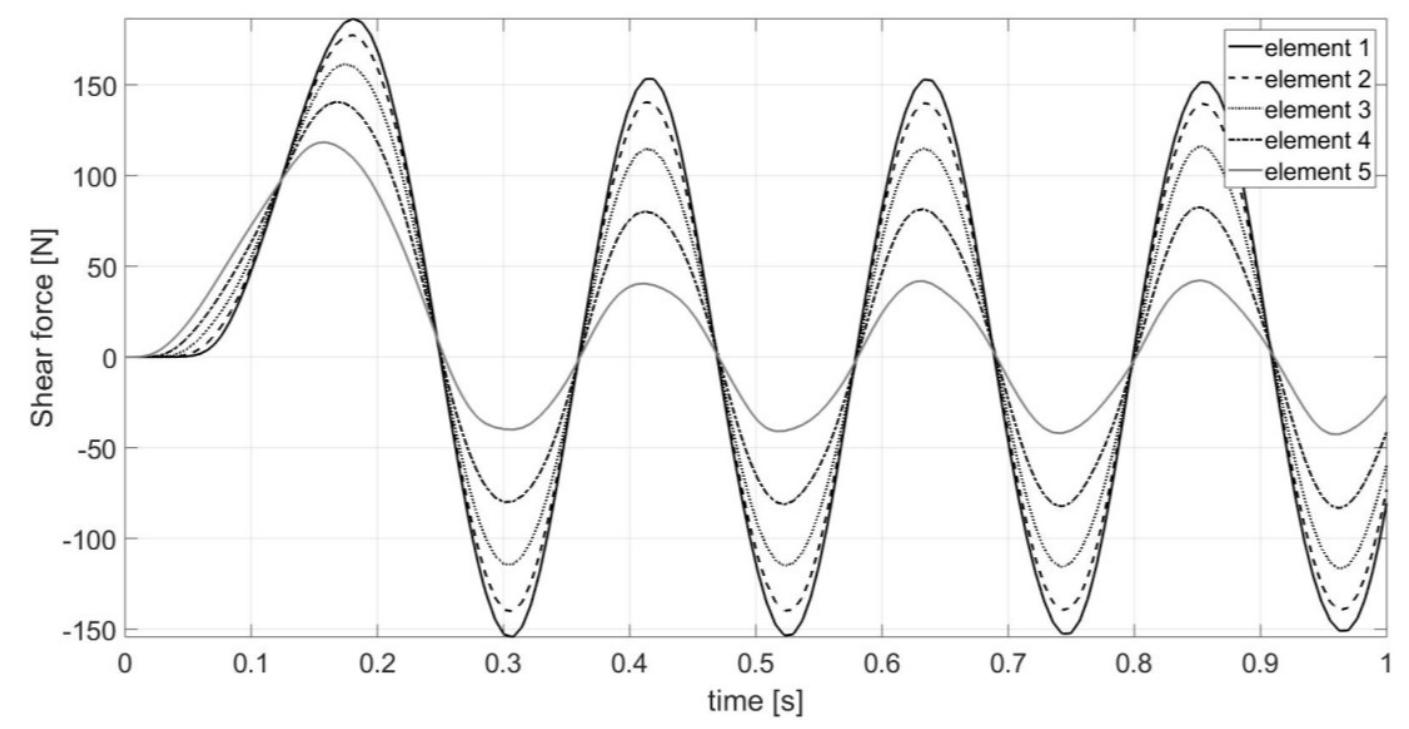

Figure 4.6. Time history of the element in-plane shear force due to a 1 minus cosine shock of 0.28 seconds. 


\subsection{RESULTS}

A comparison between the five different definitions of modal participation fractions is presented in Figure 4.7. The steady-state $L_{i}^{s t}$, transient $L_{i}^{t s}$, transient- analytical solution $L_{i}^{t s I I}$, are indeed estimates of the amplitude of the modal response $q_{i}(t)$, therefore the percentage of participation is well in agreement with Figure 4.5. A): the amplitude of the first mode is very large in comparison to that of the fifth mode for the given excitation frequency.

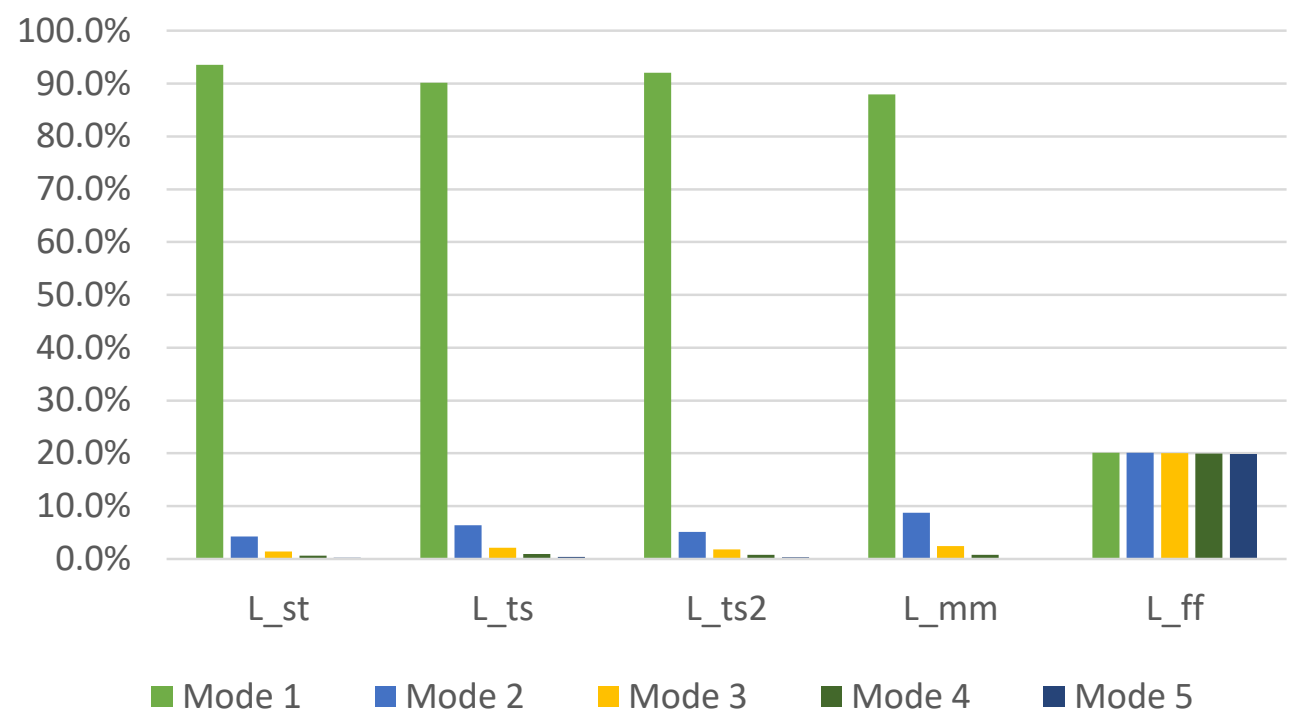

Figure 4.7. Comparison between five different definitions of Modal Participation Fractions.

The modal effective mass participation factor is also in agreement with Figure 4.5. A), a large modal effective mass indicates that a particular mode has a large modal inertial component, thus it is easier to excite. However, this metric is independent of the excitation frequency, i.e. it is constant for a given system. In this sense, the modal effective mass should not be used as a measure of the relative amplitude of the modal response, since the latter is dependent on the ratio between the natural and the excitation frequencies. 
The estimated free-free participation fractions are in disagreement with the rest of the participation fractions computed, since it indicates that the participation of all modes is almost identical. This is because, the trace of the product of two identical eigenvectors, is equivalent to the modal residue, i.e., how the $j$-th grid point reacts to a force applied in the $r$-th grid point. Therefore, this definition does not provide a real assessment on the importance of a mode, but rather how much the grid point $j$-th would react to a force applied on itself.

Using the previous results of the steady-state $L_{i}^{s t}$, the transient $L_{i}^{t s}$, and the analytical $L_{i}^{t s I I}$ participation fractions, the peak shear force at each element can be approximated using equation 4.24. The results are presented in Table 4.1, where it is clear that the analytical $L_{i}^{t s I I}$ approach yields very accurate results.

\begin{tabular}{|} 
Table 4.1. Estimation of the peak load for an undamped 1-cosine pulse forcing function. \\
\hline Element & Actual peak load & $L_{i}^{\text {St }}$ & $\%$ \%error & $L_{i}^{t s}$ & $\%$ error & $L_{i}^{t s I}$ & $\%$ error \\
\hline 1 & 186.4156 & 149.4080 & $-19.852 \%$ & 189.3272 & $1.5619 \%$ & 185.7747 & $-0.3438 \%$ \\
\hline 2 & 177.3955 & 141.8315 & $-20.048 \%$ & 157.5947 & $-11.1619 \%$ & 177.4305 & $0.0198 \%$ \\
\hline 3 & 161.4438 & 127.0626 & $-21.296 \%$ & 158.8907 & $-1.5814 \%$ & 161.3123 & $-0.0815 \%$ \\
\hline 4 & 140.5757 & 105.8503 & $-24.702 \%$ & 175.6759 & $24.9689 \%$ & 138.9849 & $-1.1317 \%$ \\
\hline 5 & 118.3022 & 79.2702 & $-32.993 \%$ & 95.8039 & $-19.0176 \%$ & 112.0805 & $-5.2591 \%$ \\
\hline
\end{tabular}

The percentage error of $L_{i}^{s t}$ is due to the omission of the transient terms; the load recovered corresponds, in fact, to the actual steady-state shear force. On the other hand, the percentage error associated with $L_{i}^{t s}$ was found to be significantly larger. The implicit assumption that all the modes 
are in-phase, creates a tendency to over or under estimate the actual magnitude of the peak physical response and therefore the peak load.

Finally, the participation fraction of each mode to the internal load was estimated using equation 4.25 , in combination with equations $4.16,4.18$, and 4.22 . The modal strain energy was also computed using equation 4.36. To compare this set of results, the values of each matrix were normalized with respect to the component with the largest magnitude and the results are displayed in Figure 4.8.
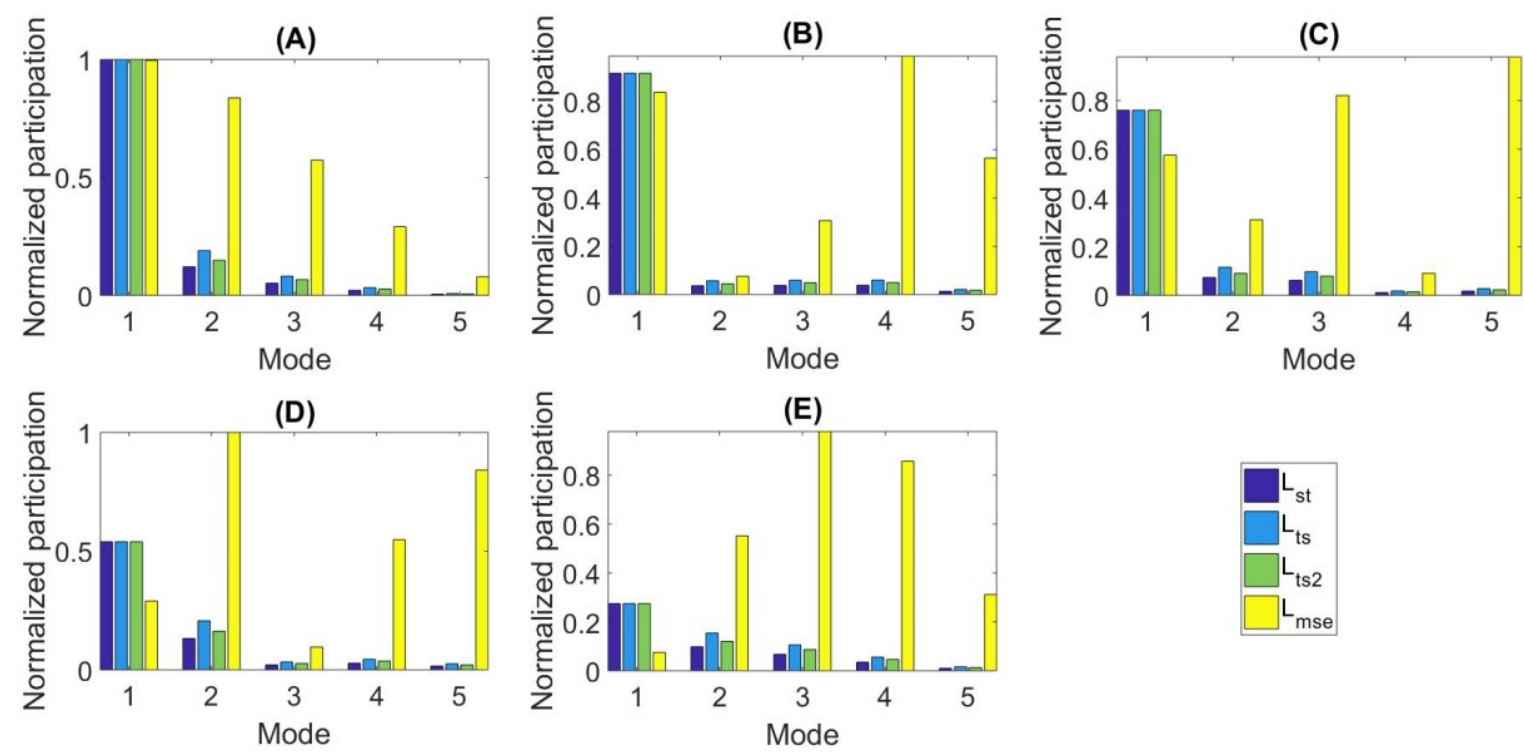

Figure 4.8. Normalized element in-plane shear force modal participation fraction: A) element 1; B) element 2; C) element 3; D) element 4; E) element 5.

In Figure 4.8, the participations estimated using equations 4.16, 4.18, and 4.22, along with equation 4.25, are shown. They are well in agreement with each other. However, the participation of the modes estimated using the modal strain energy approach differs greatly. 
Equation 4.25, is a summation of the product of two terms: firstly, $\Gamma_{i} D_{i}$ is a measure of the amplitude of the modal response; secondly the term $\Sigma_{i}^{e}$ represents the difference between the relative amplitudes of the grid points for a particular mode, the smaller the difference, the smaller the overall participation of such mode. This is the case of element 5 as seen in Figure 4.8. Though the first mode still dominates, it does it in a smaller proportion whereas the rest of the modes gain importance. In contrast, the magnitude of the load in element 1 is dominated by the first and second modes; such that any structural modification that significantly affects such modes, will produce significant effects on the peak load of element 1.

According to the modal strain energy, in Figure 4.8, it appears that the higher frequency modes are more relevant to the overall response. Keep in mind that the definition given by equation 4.36 indicates the ratio between the modal strain and the modal kinetic energies of the system. Thus, a small value indicates that the kinetic energy (i.e., the inertial component) is more relevant to the response than the quasi-static strain energy.

For example, in the case of 5-th element, the $3^{\text {rd }}$ mode carries a larger strain energy, therefore, any modification that impacts the third mode shape, $\varphi_{3}$, (but doesn't necessarily modifies the kinetic energy), would largely impact the response of element 5 . On the other hand, any modification on the first mode shape, $\varphi_{1}$, will not affect significantly its response. Instead if its natural frequency were to be modified $\omega_{n_{1}}$, a large change in the kinetic energy would be expected and thus in the magnitude of the load in element 5 . 


\subsection{BEST CHOICE OF METRIC FOR THE SURROGATE-AIDED RESPONSE ALGORITHM}

The first class of modal response participation factors (SMPF) have the potential to be used as reanalysis tools, since they allow to estimate the after-modification response without the need to solve the equations of motion. When the excitation force is periodic, and the interest lies in solving for the steady-state response, the $L_{i}^{s t}$ fraction provides the most accurate results. However, when the peak load is of interest, $L_{i}^{t s I I}$ should be the used, since it provides excellent results.

The second class of modal participation factors are independent of the excitation and depend solely on the mass and stiffness distribution, in this sense, this type of factors should not be used as a measure of the relative amplitude of the modal responses, since these are a function of the excitation frequency. Instead, their application should be restricted, for example, in the case of the modal effective mass fraction, to determine the number of modes to be retained in the analysis.

The modal strain energy, on the other hand, is an inexpensive expression that relates the mass and stiffness distribution of the structure to its modal characteristics. This expression can be useful to visualize how modifying the physical properties at the element level can impact the behaviour of a specific mode.

Based on the results presented in the previous subsection, the obvious choice of metric is that of the SMPF. However, a key requirement for the implementation of the SMPF is for the equation of motion in the modal domain to be uncoupled, which in the case of aeroelastic models, does not occur due to the addition of the matrix of aerodynamic influence coefficients.

Thus, a first step to implement the SMPF is to uncouple the aeroelastic equations of motion. This step falls out of the scope of this project. Instead, a correlation model between the modal strain 
energy coefficient (MSEC), as presented in equation 4.37, and the peak aeroelastic load at element level were selected as the metric of choice.

Though, far from ideal, this choice of metric provides a quick guess of the model's behaviour during optimization. The obvious drawback is the need to construct the correlation model prior to the actual structural optimization and the exploration of the design space. The implementation of this metric will be further addressed in the next chapter. 


\section{IMPLEMENTATION OF THE SURROGATE-AIDED RESPONSE ALGORITHM}

The code was implemented in Matlab, where the Design and Analysis of Computer Experiments (DACE) toolbox [72] was adopted for the construction of the surrogate model. This tool box is a free-source code for the Kriging interpolator and the Latin Hypercube. The block diagram of the algorithm is shown in Figure 5.1 and the core of the code is presented in Appendix I.

The algorithm starts with the definition of a grid-like design space, followed by the selection of the initial sampling points using the Latin Hypercube scheme. Then, the MSEC of all the structural elements selected for monitoring are computed using equation 4.37. An estimated incremental load $(\Delta L)$ is calculated based on the previous and current values of the MSEC and a polynomial.

In fact, a family of polynomials is constructed prior to the exploration of the design space. At this initial stage, there is no information about how the optimization process will modify the maximum/minimum monitored loads. As a matter of fact, the location of the maximum/minimum response is yet unknown. However, global information can be drawn from local data, i.e. from a single point, randomly selected, in the design space. By using a metric that allows us to correlate the change in structural parameters to the variation of the monitored loads, namely the MSEC. For this purpose, a "dummy" optimization is performed about a single point in the design space, a correlation model is built, and the resulting polynomials fed into the main program.

When the estimated incremental load, $\Delta L$, is larger than a user specified threshold, $\gamma$, the design space needs to be re-explored in search of the minimum and the maximum loads; else, if the incremental load, $\Delta L$, is smaller than $\gamma$, the optimization process continues. Usually, variations 
less than $5 \%$ of the monitored loads are deemed negligible. However the choice of $\gamma$ is left to the user's free will.

Since the design space is shared by all the monitored elements and all the monitored loads, once the exploration of the design space gets triggered due to $\gamma$ being surpassed, all the response sample vectors, $\boldsymbol{Y}(\boldsymbol{X})$ are populated simultaneously. Maximizing the use of the information that can be extracted by computing a single sample point.

The sampled data now enters the EGO algorithm block. Here, a cross-validation diagnosis is run per surface response. If the diagnosis fails, the program will launch a warning, suggesting to either perform a data transformation or to increase the initial number of sample points. Then, the Expected Improvement locates a new observation point, runs the simulation, and collects the results. This process repeats itself until the Expected Improvement of the function is smaller than a selected threshold $\left(E_{I}\right)$. The $\mathrm{E}$ threshold can be chosen to be equal or smaller than $1 \%$ of the current maximum/minimum value of the interpolated response surface [10]. After the interpolation is completed, the global $\mathrm{min} / \mathrm{max}$ are located, and the time consistent loads recovered. 


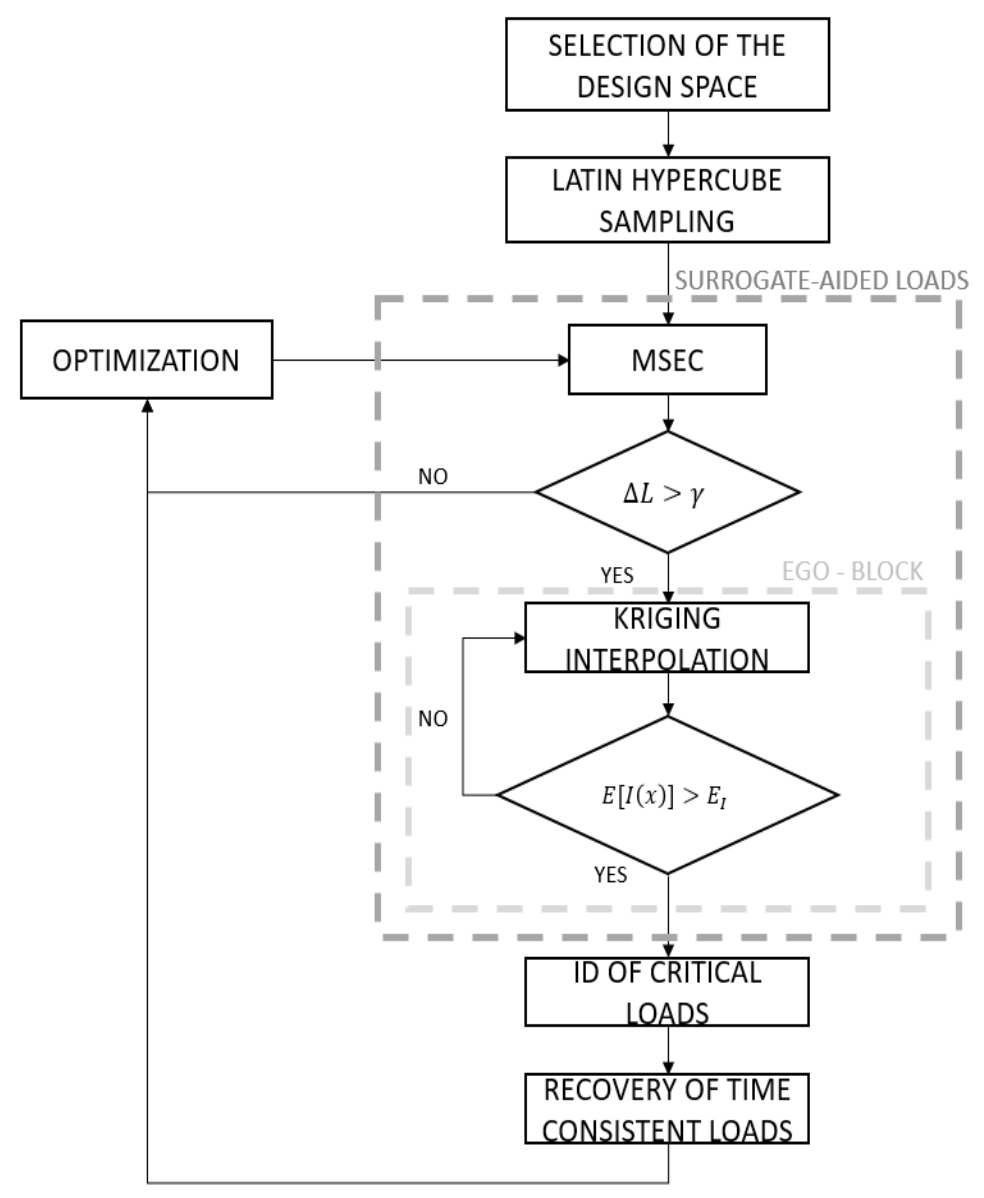

Figure 5.1. Overview of the Algorithm.

\subsection{ELEMENT MODAL STRAIN ENERGY CORRELATION FUNCTION APPLICATION}

The question of how sensitive the dynamic loads are to changes in design variables, is a cumbersome one. Though their study may offer a powerful tool, in practice they are often too expensive to compute. Since, as in the case of aeroelastic models, there is no simple analytical equations.

Though the question of which variables may induce large changes in the response remains unanswered, other possibilities can be explored to assess when a significant change has occurred. 
To that end, the use of a correlation model, based on the element modal strain energy factor, is proposed.

Such idea was drawn from the concept of modal participation factors, as presented in the previous chapter, which aims to determine inexpensively the relative importance of each mode retained in the dynamic solution and how structural modifications affect their dynamic response.

Thus, a dummy optimization is run firstly, as shown in Figure 5.2, to estimate the MSEC, as per equation 4.37 and the response of interest around a single point in the sky for a set of stations (or elements). Then, a polynomial function, that best fits the data, is used to create a correlation model for each station and each response of interest.

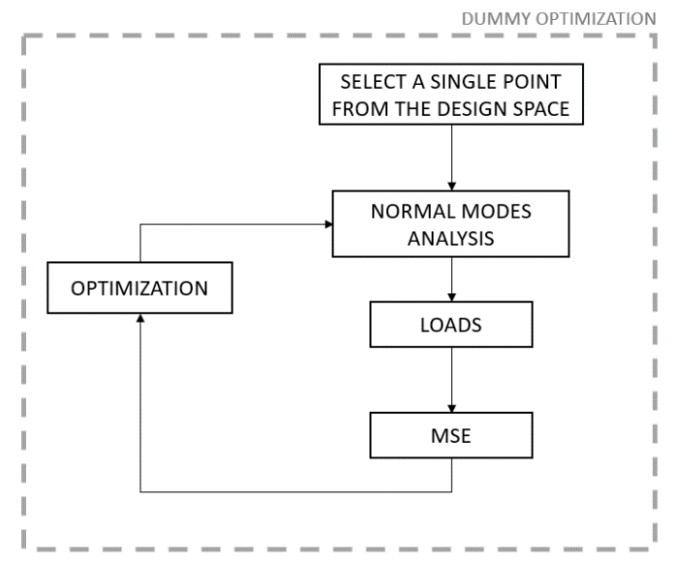

Figure 5.2. Block diagram of the dummy optimization, required to construct the correlation model between the MSEC, as per equation 4.37, and a response of interest.

This model is not intended to be used for extrapolation, nor to predict the magnitude of the load at any other point in the design space. Instead, local information from a single sample point is extracted and one can expect that the rest of the design space will behave in a similar fashion, i.e., if the MSEC varies, then the monitored load is expected to also vary around the whole design 
space, except there is no certainty about how much did the magnitude of the maximum or the minimum load vary or if they surpassed the $\gamma$ threshold. There is only certainty that in at least one point in the design space the change in the load did or did not exceeded $\gamma$.

\subsection{EGO BLOCK APPLICATION}

To test the performance of the implemented EGO algorithm, two response surfaces are used as examples. The first one, shown in Figure 5.3, is the normalized envelope of the maximum out of plane bending moment of the main case study: aeroelastic model of a Bombardier Aerospace Platform (in the next chapter, more details about it will be presented). The second one, Figure 5.6, is a multimodal function given by equation 5.1 .

$$
f\left(x_{1}, x_{2}\right)=x_{1}^{3} \sin \left(x_{1}\right)-x_{2}^{2}
$$

The EGO algorithm is capable of handling very complex multimodal function. However, there is an important trade-off between the cost of exploring such design space and the level of accuracy desired. For example, in the case of Figure 5.3 a total of 207 points out of 627 (about 33\%) were used to reconstruct the surface response, where the percentile error was bounded between $[-4.5$, $+4.5]$ as shown in Figure 5.5. In contrast, Figure 5.6 was reconstructed using 39\% of the points and its percentile error is bounded between $[-10,+10]$, as shown in Figure 5.8.

The QQ plots shown in Figure 5.4 and Figure 5.7, present the outcome from the cross-validation diagnosis for both test functions. Though the diagnosis passed, the scattered points indicate that some of the residuals do not follow a normal distribution (if they did, the scatter points would lie along the dotted line), thus the error in the response surface can be attributed to the lack of normality in the residuals. 


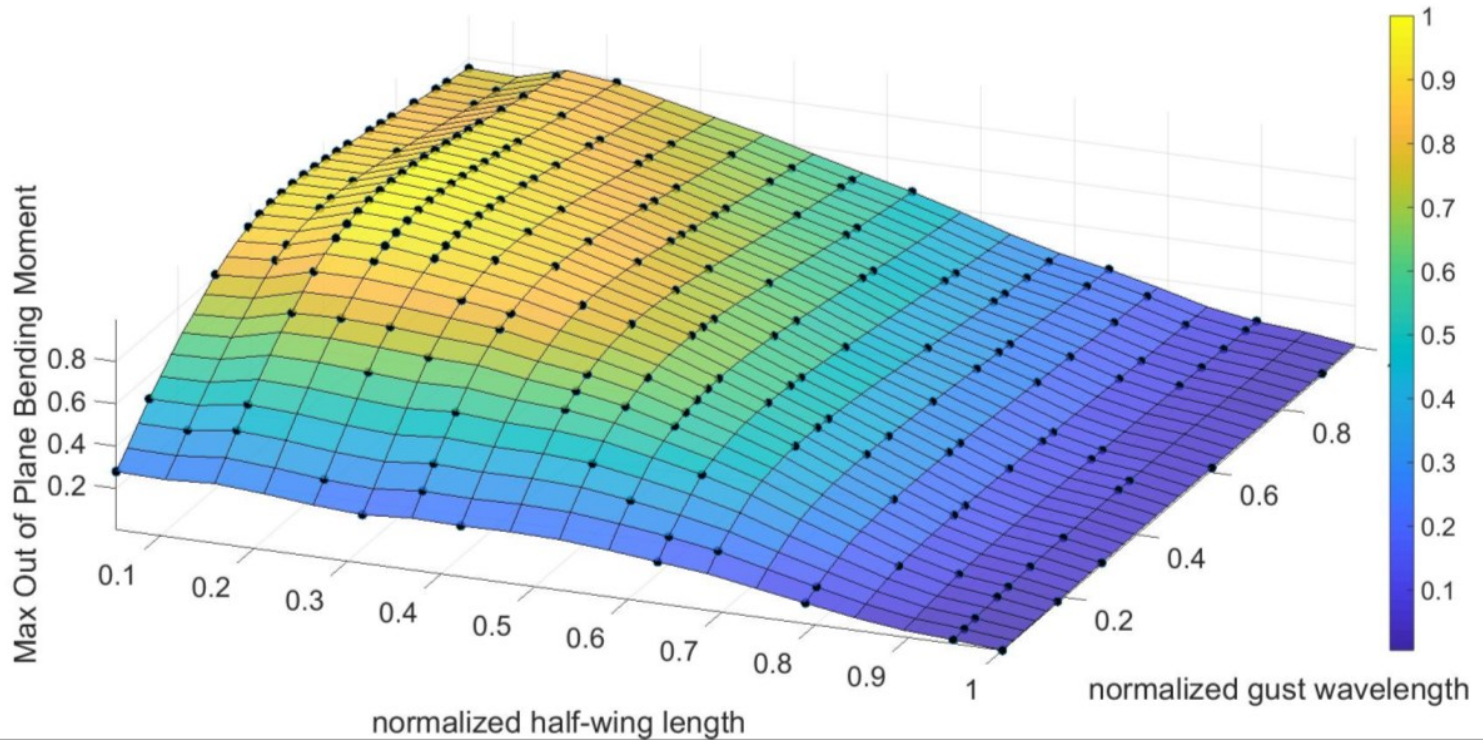

Figure 5.3. EGO Implementation: Estimated maximum out of plane bending moment (normalized) as a function of gust wavelength and half-wing length. The scatter points are the observations (207 points out of 627) used to reconstruct the surface response.

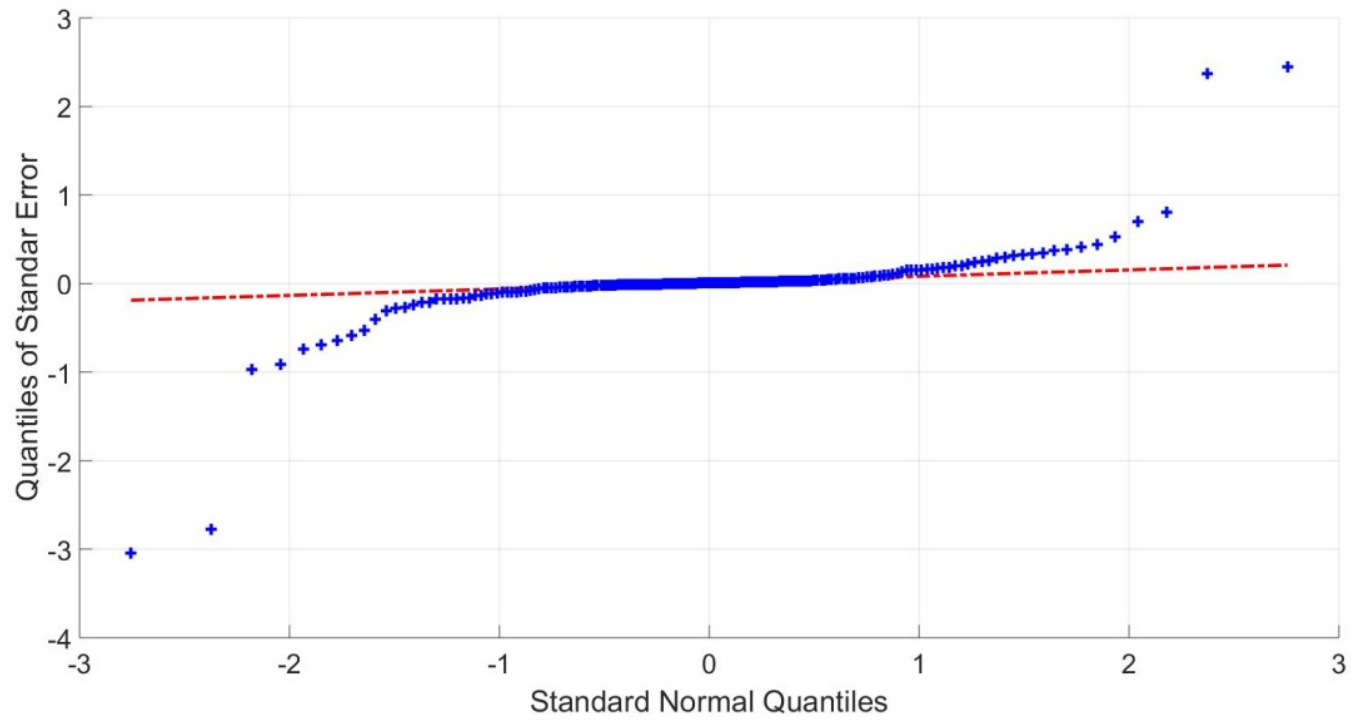

Figure 5.4. QQ plot of the standard error for the estimated maximum out of plane bending moment (normalized) as a function of gust wavelength and half-wing length. 


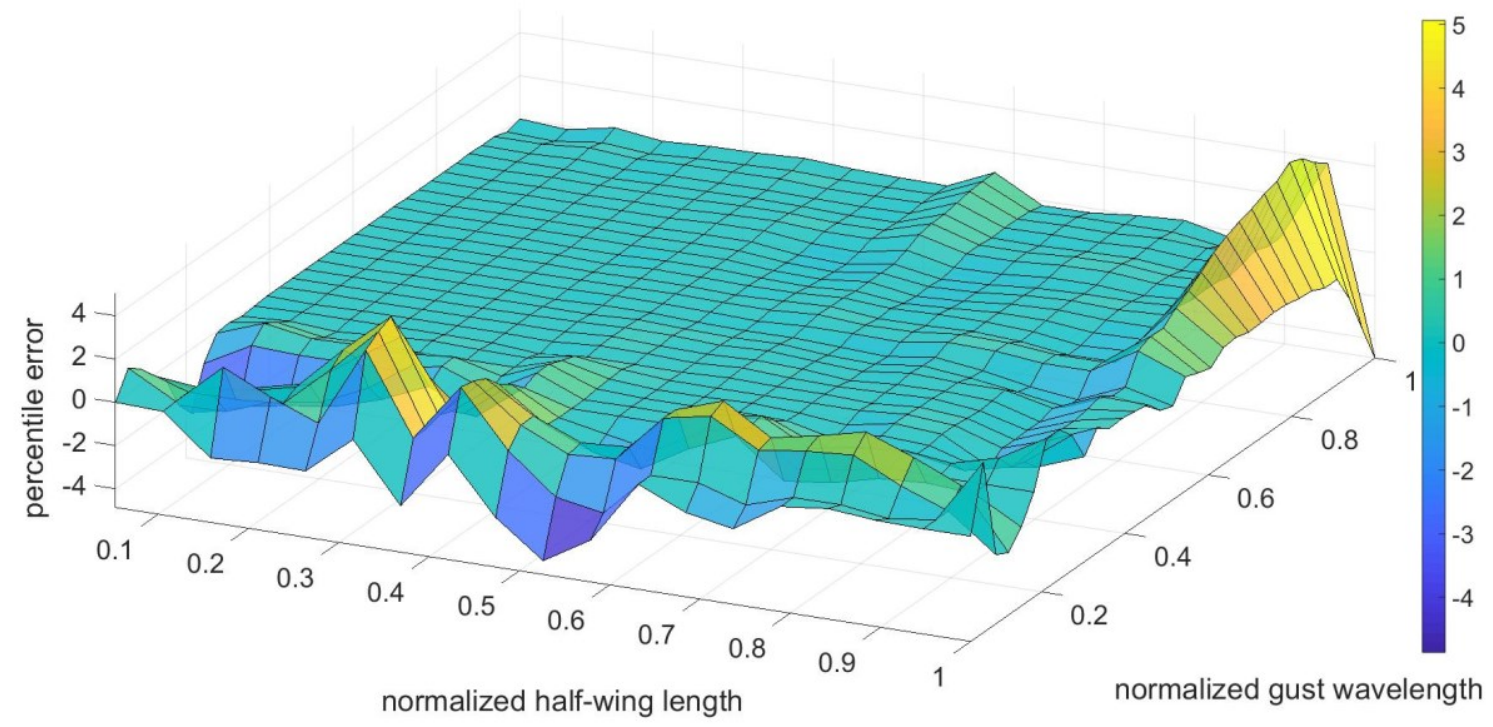

Figure 5.5. True percentile error between the real and the estimated maximum out of plane bending moment (normalized) as a function of gust wavelength and half-wing length.

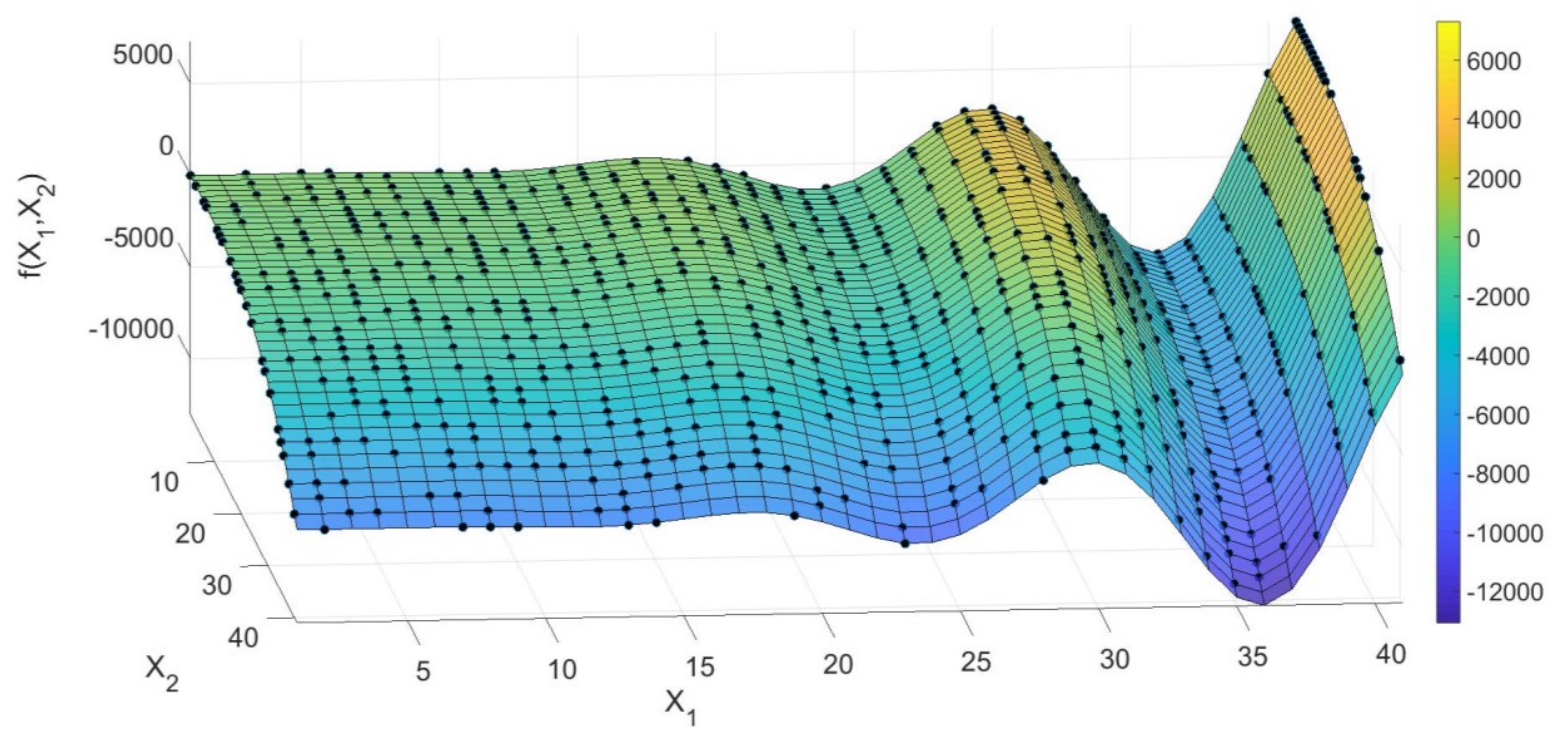

Figure 5.6. EGO Implementation: Estimated multimodal test function. The scatter points are the observations (664 points out of 1681) used to reconstruct the surface response. 


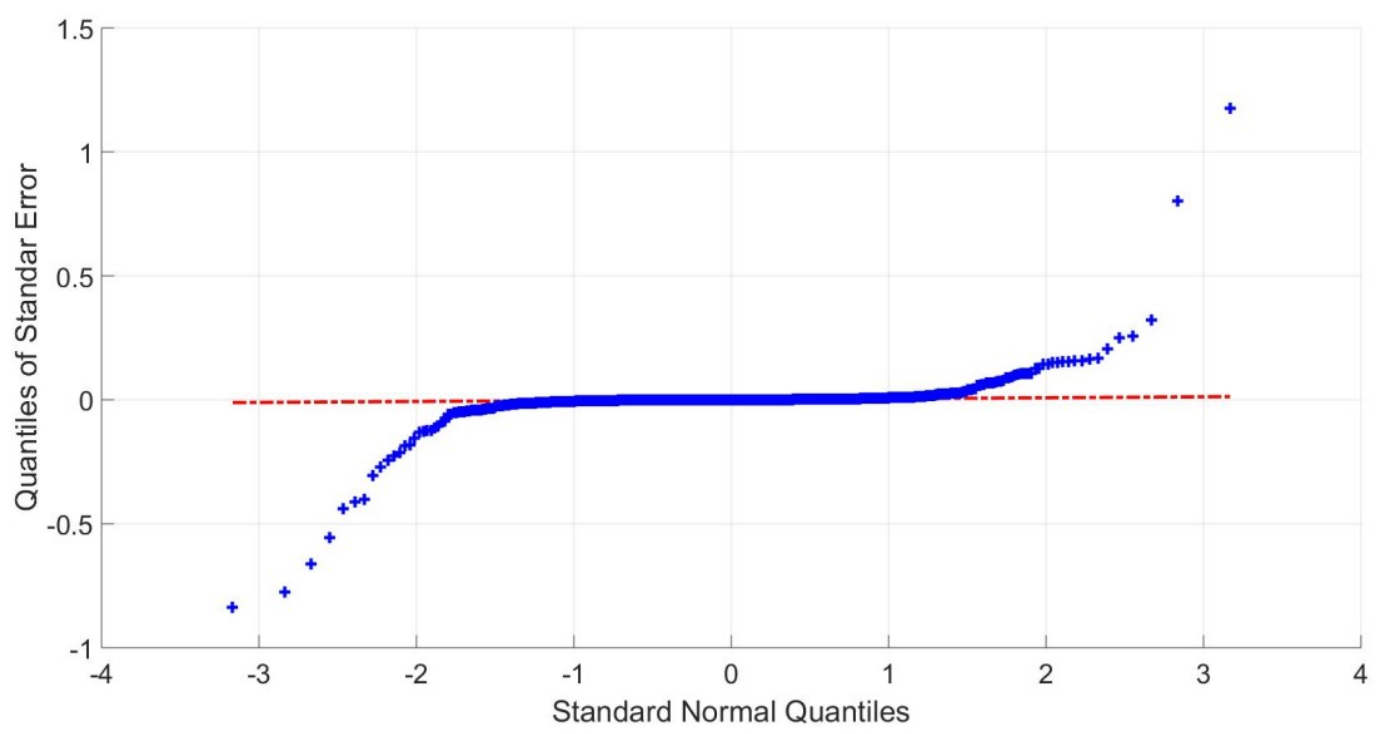

Figure 5.7. QQ plot of the standard error for the estimated multimodal test function.

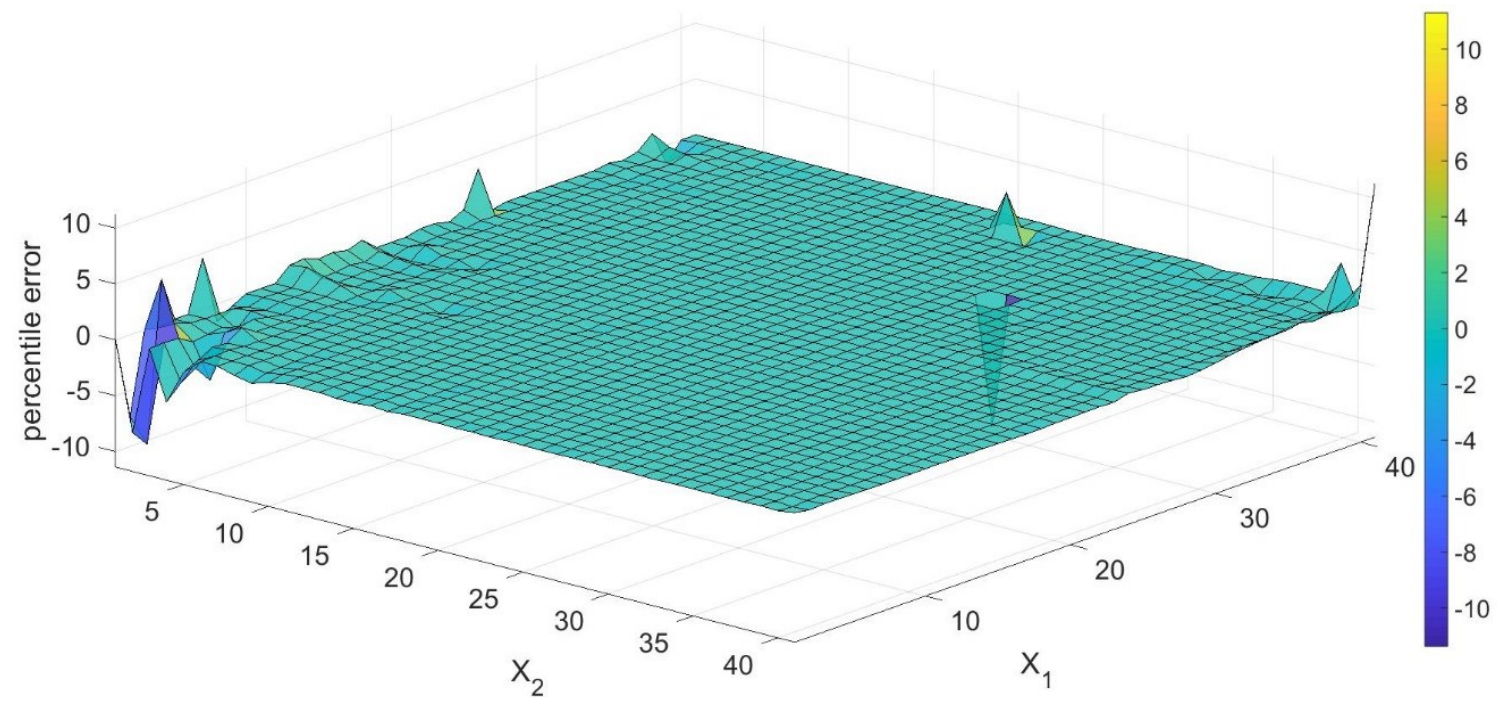

Figure 5.8. True percentile error between the real and the estimated multimodal test function. 


\section{CASE STUDY}

To test the algorithm, the aeroelastic model of a Bombardier Aerospace platform, shown in Figure 6.1 , is used. The goal, in this scenario, is to locate the global max/min incremental load due to a dynamic discrete gust for a range of altitudes and Mach numbers. Each combination of altitude and Mach number will be referred herein as a "point in the sky" and this comprises the design space.

At each point in the sky, the FAR-25 [5] regulations require to investigate the dynamic behaviour of the aircraft due to a family of discrete gust, with varying wave lengths from 60 to $700 \mathrm{ft}$., where the design velocity of the gust is altitude dependent. Gust wavelength increments of $20 \mathrm{ft}$. were considered at each sampled point. Therefore, the dynamic response of the aircraft is evaluated for 33 gust cases. Then, the maximum response from this family of gust cases is identified and its magnitude stored in the sample response vector $\boldsymbol{Y}(\boldsymbol{X})$.

Here, 6 loads were monitored: maximum/minimum out of plane bending moments $(\mathrm{M} / \mathrm{m}-\mathrm{OPB})$, the maximum/minimum out of plane shear forces (M/m - OPS) and the maximum/minimum torsional moments $(\mathrm{M} / \mathrm{m}-\mathrm{T})$; at 5 elements distributed over the left side half-wing. This means that a total of 30 response surfaces were constructed each time the design space was explored. The structural loads were all computed using Nastran solution 146.

For simplicity, in this study the optimization block was replaced with a set of parametric variations of the stick models' mass and stiffness. It mimics the change in structural properties due to skin thickness reduction. To this end, the height of the beam elements composing the wing were varied 
in small increments and its geometrical characteristics were correlated to structural stiffness's and weights. In total seven iterations were performed.

To compare the performance of the proposed methodology, a full factorial search was also performed. In the following section the results are discussed.

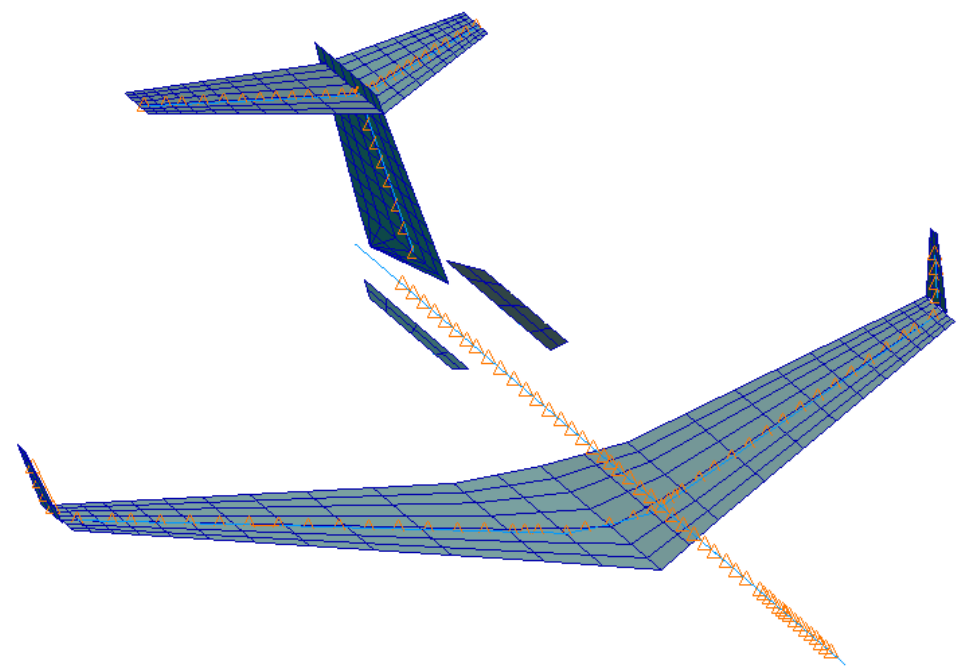

Figure 6.1. Aeroelastic model of a Bombardier Aerospace Platform.

\subsection{NUMERICAL MODEL PARAMETERS}

An aeroelastic model was provided by the industrial partner Bombardier Aerospace. In the following subsection, some of the numerical values used in this study that are deemed necessary for the understanding of the reader are presented. Information relevant to the design, such as stiffnesses, payload, take-off weight, structural mass, etc., were omitted to protect the integrity of the design. 


\subsubsection{Parametrized mass and stiffness variations}

The relationship between structural mass and stiffness is certainly complex, since the wing itself is comprised of multiple components such as ribs, skin, spars, gears, joints, etc. This intricate analysis falls out of the scope of this project and instead, for simplicity, a set of preliminary results, provided by Bombardier Aerospace, that relate the variations of structural mass to variations of element stiffnesses were considered [104]. Here, the material properties remained constant throughout the study.

The mass and stiffness variations are restricted to the aircraft wing box, where these variations are the result of skin thickness variation. The data compiled is shown in Figure 6.2 through Figure 6.5 where the percentile change in structural mass is related to the percentile change of axial, shear and torsional stiffness's.

Using this data, a multivariable polynomial function was estimated to relate the percentile change in structural mass with respect to percentile changes of element stiffness properties.

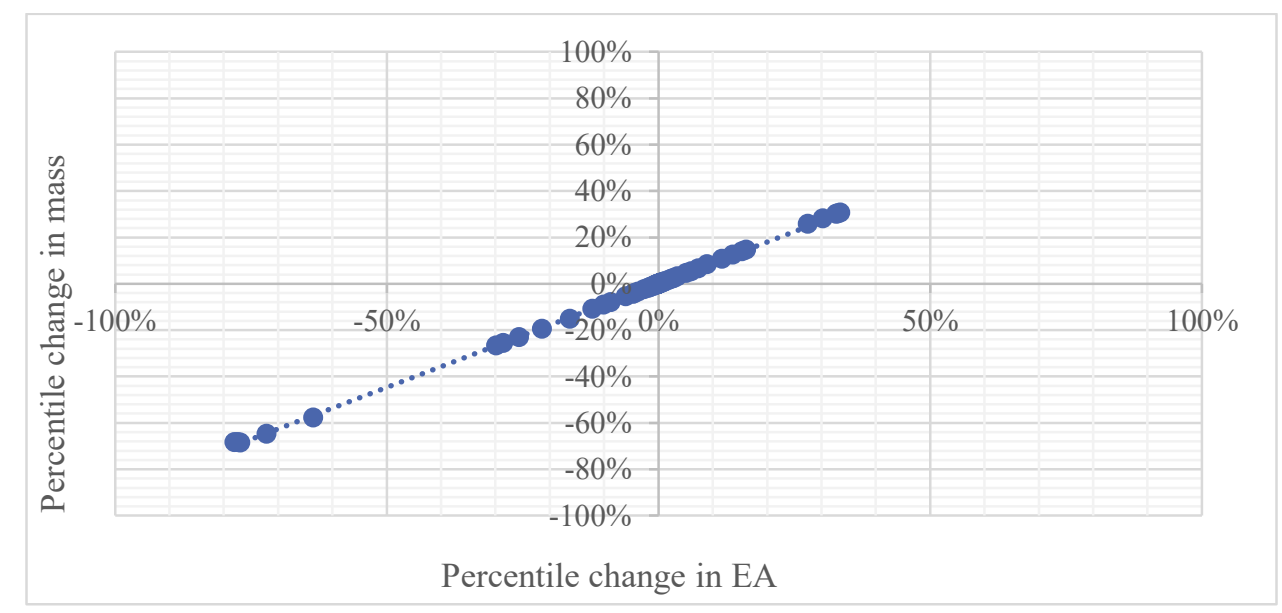

Figure 6.2. Percentile change in mass versus percentile change in axial stiffness (EA) as a result of skin thickness reduction in the wing box. Data retrieved with permission of Bombardier Aerospace [104]. 


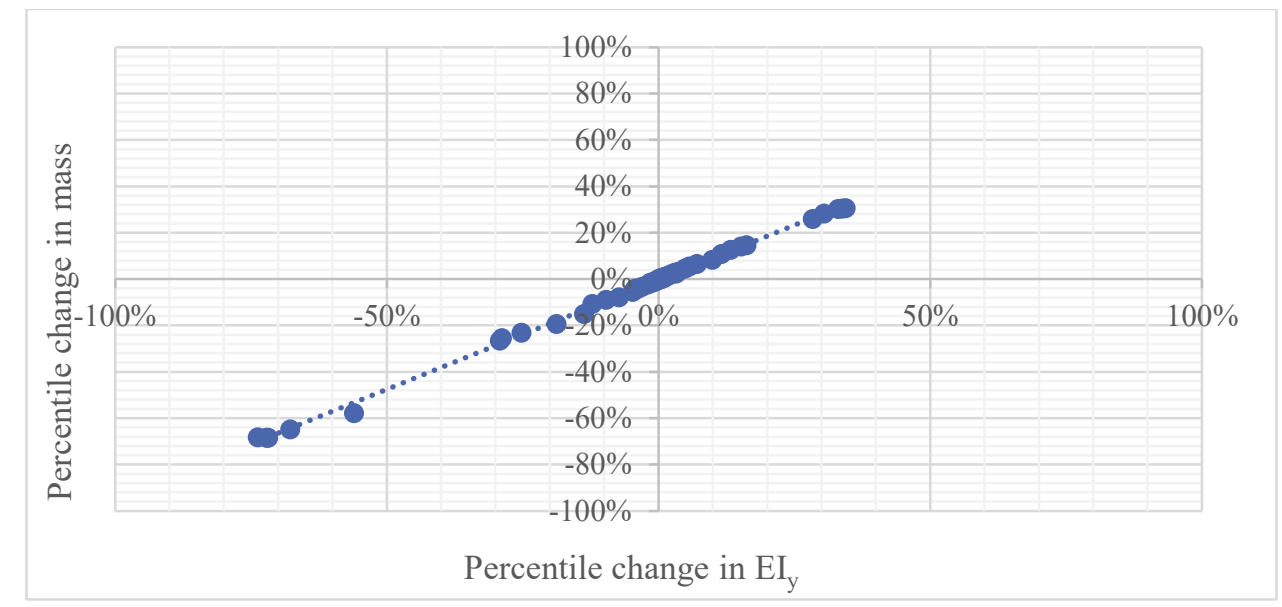

Figure 6.3. Percentile change in mass versus percentile change in shear stiffness about the y axis (EIy) as a result of skin thickness reduction in the wing box. Data retrieved with permission of Bombardier Aerospace [104].

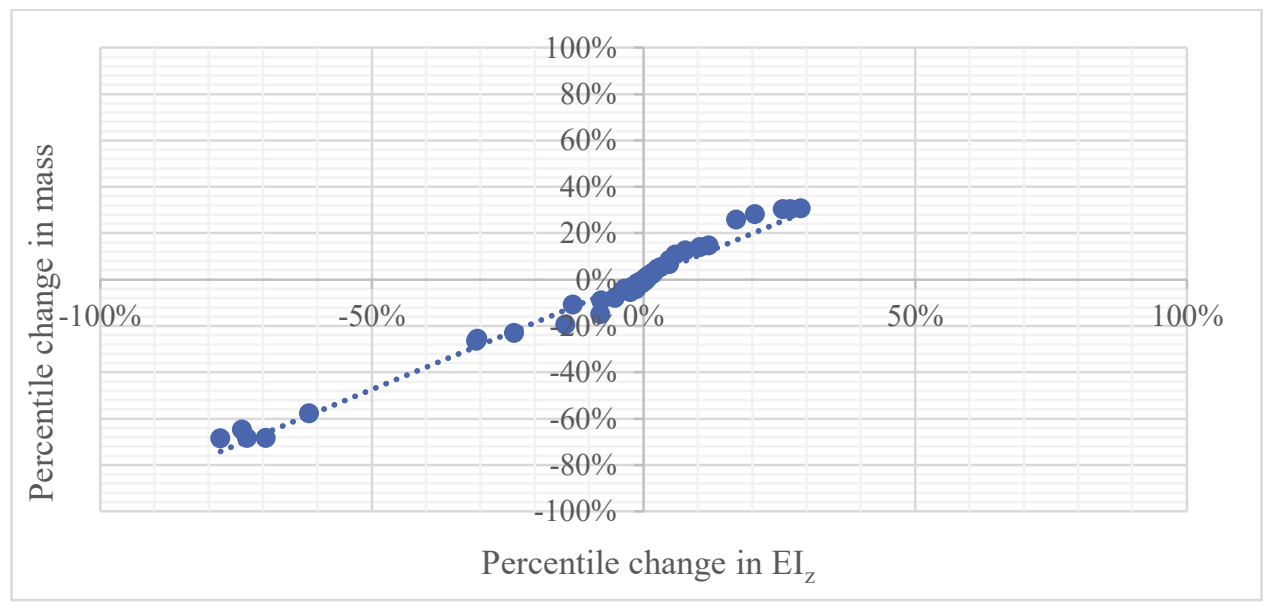

Figure 6.4. Percentile change in mass versus percentile change in shear stiffness about the $\mathrm{z}$ axis (EIz) as a result of skin thickness reduction in the wing box. Data retrieved with permission of Bombardier Aerospace [104]. 


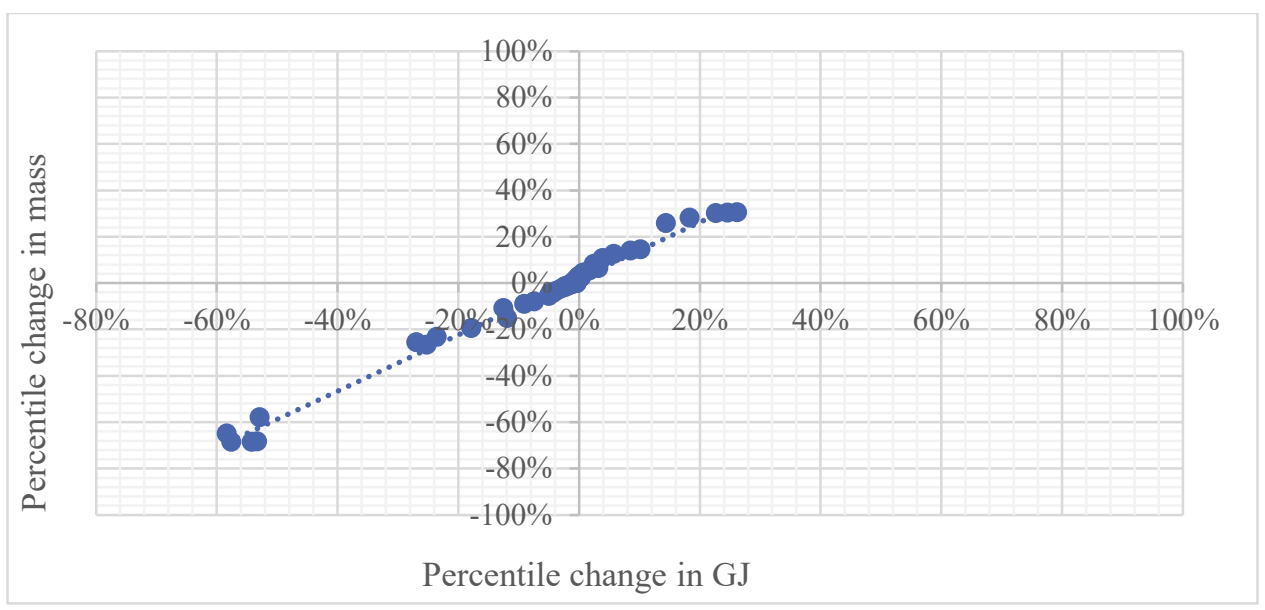

Figure 6.5. Percentile change in mass versus percentile change in torsional stiffness (GJ) as a result of skin thickness reduction in the wing box. Data retrieved with permission of Bombardier Aerospace [104].

From the baseline model, the equivalent cross-sectional dimensions of the beam-elements that compose the stick model were computed as follows:

$$
\begin{gathered}
w=\left(\frac{144 I_{y}^{3}}{I_{z}}\right)^{1 / 8} \\
h=\frac{12 I_{y}}{w^{3}} \\
J=w h^{3}\left[\frac{1}{3}-\left(\frac{21 w}{100 h}\right)\left(1-\frac{h^{4}}{12 w^{4}}\right)\right]
\end{gathered}
$$

Where $w$ is the width and $h$ is the height of the beam element. $I_{z}$ and $I_{y}$ are the second moments of area and $J$ is the second polar moment of area. The optimization block of the present study was then replaced by height and width variations of the stick models' beam-elements, which are related to stiffness variations via equations 6.1 to 6.3 and to structural mass variations via the multivariate 
polynomial function described above. Overall, 7 iterations were performed, where the parameter $h$ was varied in small increments; emulating the behaviour of an optimizer.

\subsubsection{Design Space}

To locate the maximum/minimum dynamic out of plane bending moment $(\mathrm{M} / \mathrm{m}-\mathrm{OPB})$, the maximum/minimum dynamic out of plane shear force (M/m - OPS) and the maximum/minimum dynamic torsional moment $(\mathrm{M} / \mathrm{m}-\mathrm{T})$ due to a discrete gust incidence the dynamic response of the aircraft for a range of velocities and altitudes needs to be investigated. Additionally, as per agency regulations, a family of gusts need to be investigated per flight condition.

In this study, the design space ranges from an altitude of 3000 to $45000 \mathrm{ft}$. with increments of 3000 ft. and Mach numbers from 0.10 to 0.80 , with increments of 0.025 . This means, that in a full factorial search, a total of 435 simulations would be required to populate the design space. At each flight condition, 33 discrete gusts with wavelengths ranging from 60 to $700 \mathrm{ft}$. in increments of 20 ft. (as shown in Figure 6.6) were investigated. This information is summarized in Table 6.1.

\begin{tabular}{|c|c|}
\hline \multicolumn{2}{|c|}{ Table 6.1. Design Space } \\
\hline Flight Conditions & Range \\
\hline Altitude [ft.] & $3000-45000$ \\
\hline Mach & $0.10-0.80$ \\
\hline Gust wavelength [ft.] & $60-700$ \\
\hline
\end{tabular}




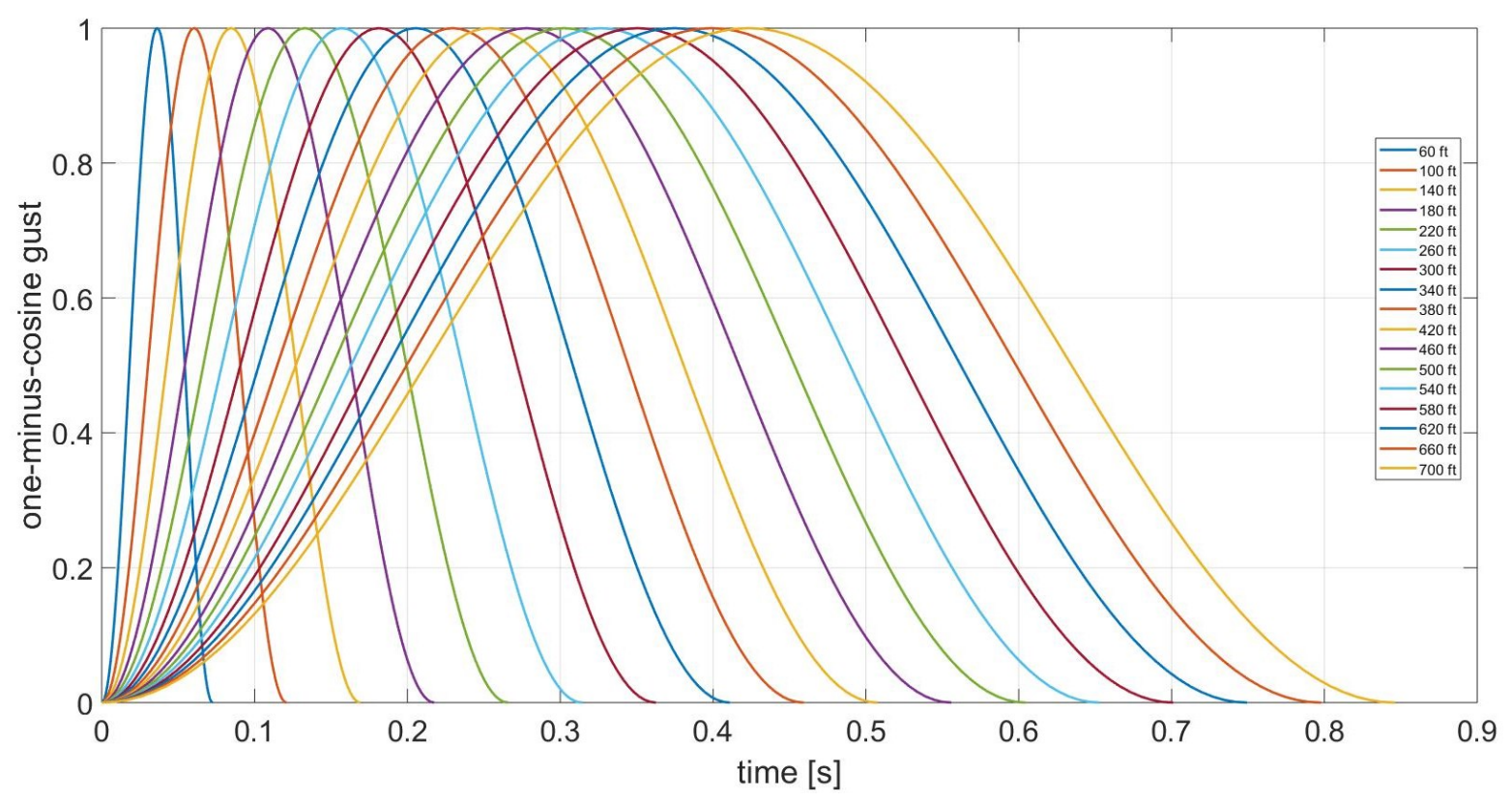

Figure 6.6. Family of gust wavelengths according to FAR25.341 regulations.

Following the mathematical models described in Chapter 2, the gust design velocity $w_{o}$, depends on two altitude-dependent variables: the gust reference speed $w_{\text {ref }}$ and the flight profile alleviation factor $F_{g}$.Their definitions are presented graphically in Figure 6.7 and Figure 6.8, respectively, as defined in [5].

The algorithm developed includes a subroutine, in which, depending on the selection of the design space, the appropriate one-minus cosine gusts are estimated. These represent the model's forcing function in the time domain. 


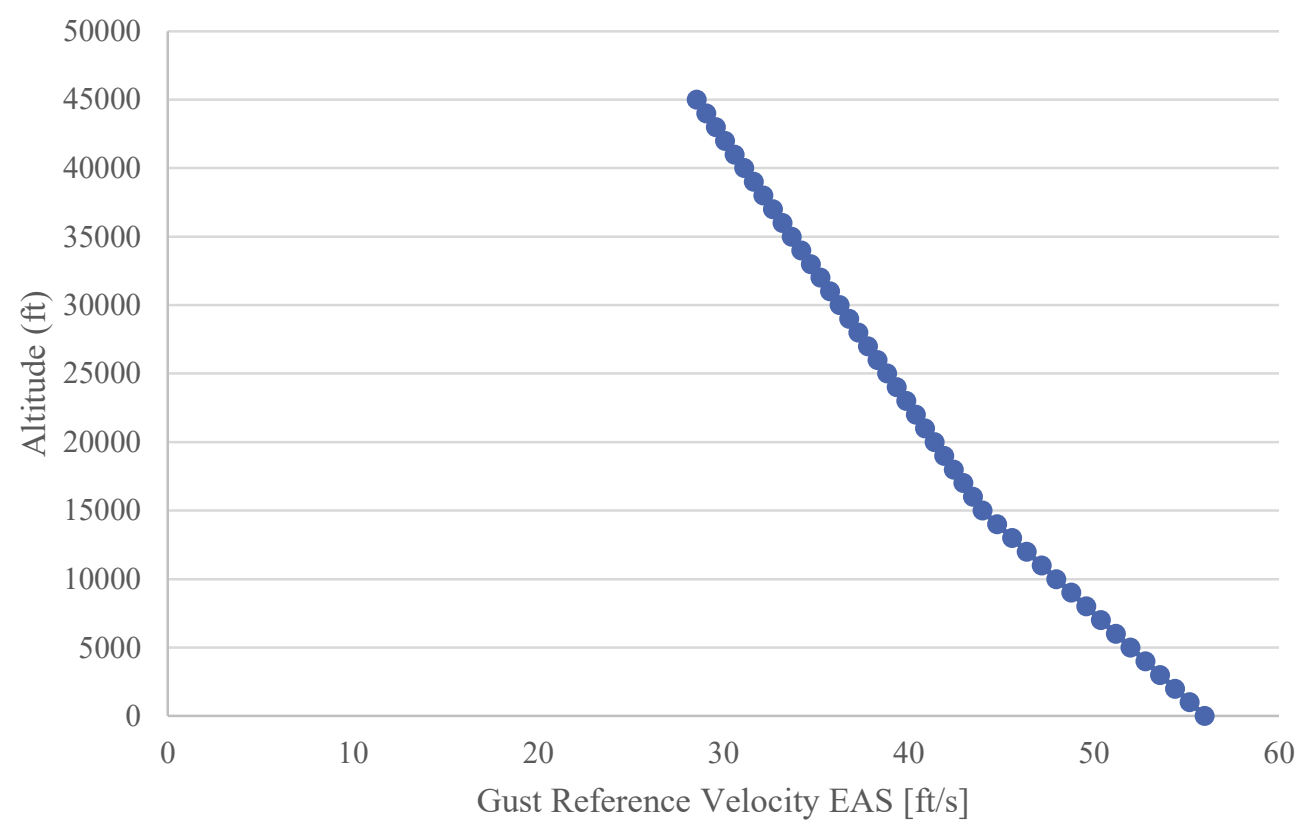

Figure 6.7. Gust reference velocity according to FAR25.341 regulations.

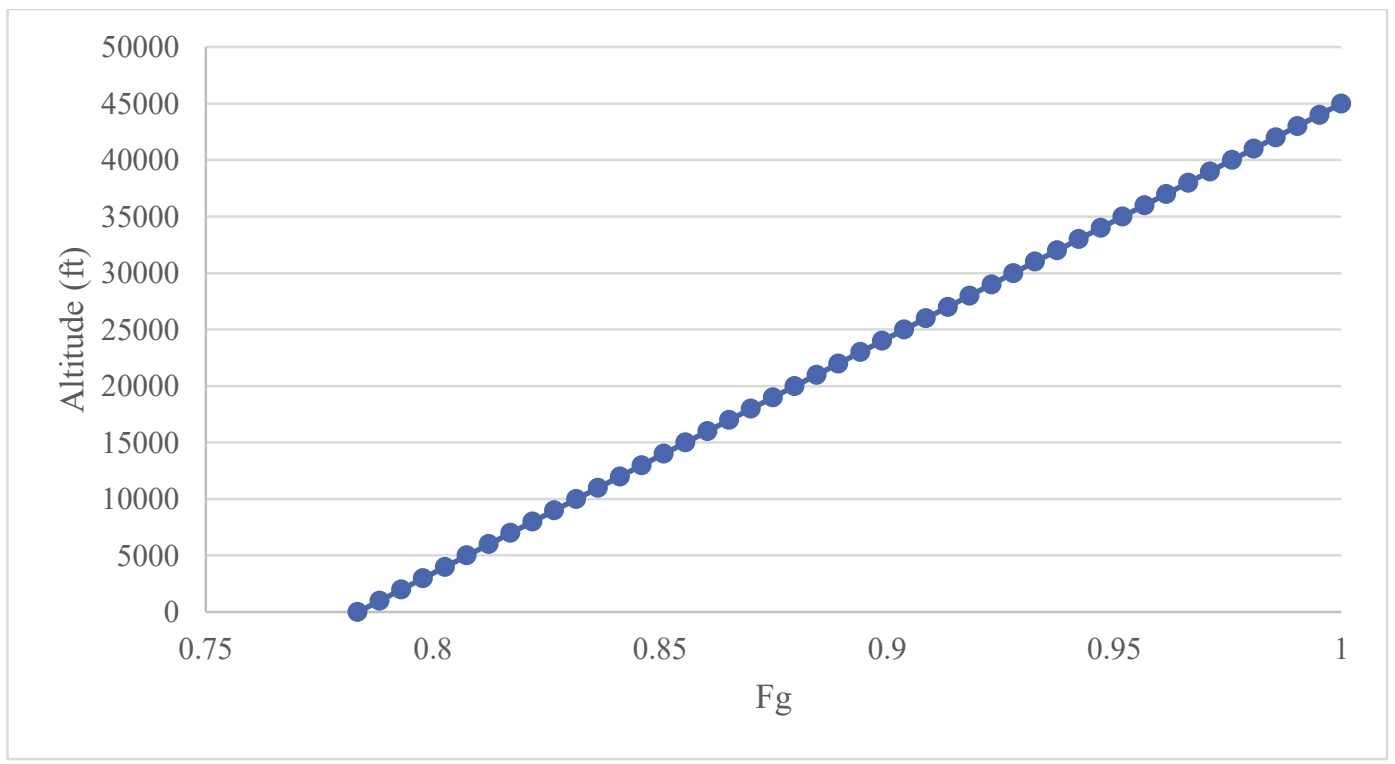

Figure 6.8. Flight Profile Alleviation Factor per FAR25.341 regulations. 


\subsection{Results}

The MSEC correlated points and corresponding polynomial fits, for five beam elements distributed through the left sided half-wing, beginning with the wing root element $(\bar{y}=0)$ and finalizing with the wing tip $(\overline{\mathrm{y}}=1.0)$ are presented in Figure 6.9 through Figure 6.13 , where each figure was normalized with respect to its own maximum scale. The normalized location of such elements are highlighted in Figure 6.14.

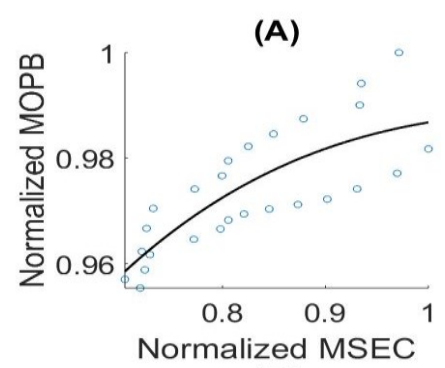

(D)

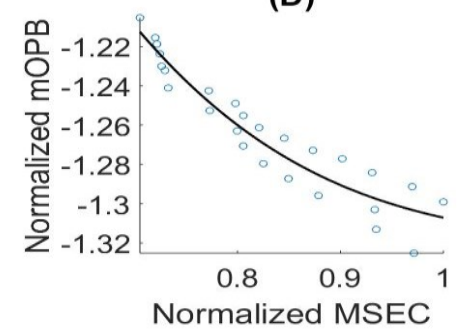

(B)

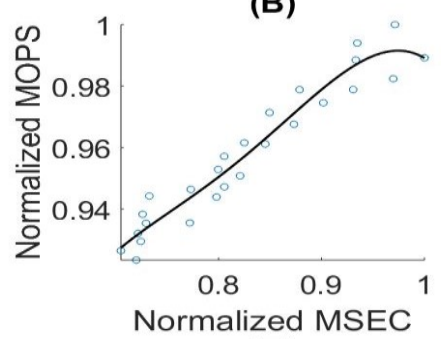

(E)

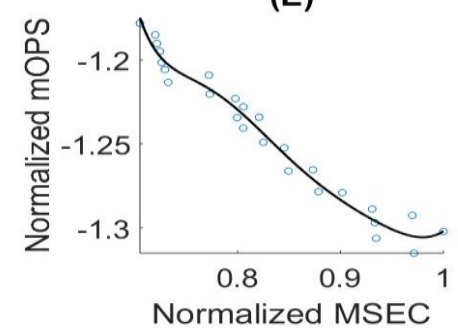

(C)

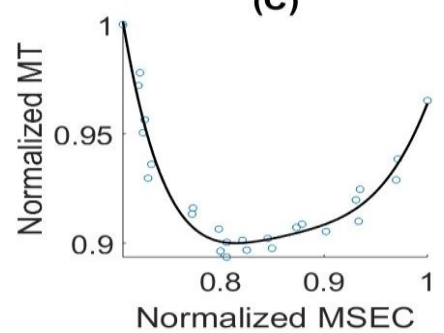

(F)

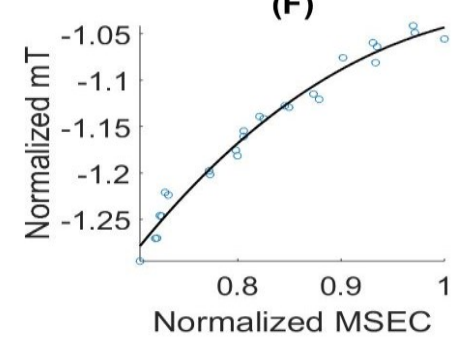

Figure 6.9. Normalized loads versus MSEC at the wing root, $\bar{y}=0$. The scatter points are the correlated data and the solid lines are the polynomial fits for the following loads: A) Maximum out of plane bending moment (MOPB); B) Maximum out of plane shear force (MOPS); C) Maximum torsion moment (MT); D) Minimum out of plane bending moment (mOPB); E) Minimum out of plane shear force (mOPS); F) Minimum torsion moment (mT). 


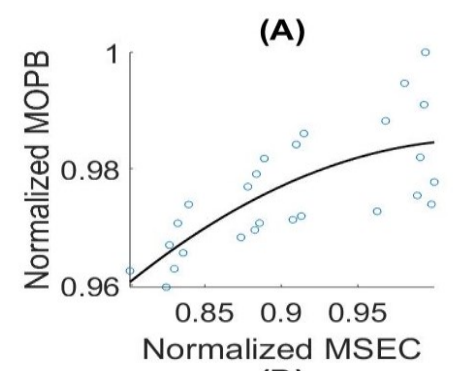

(D)

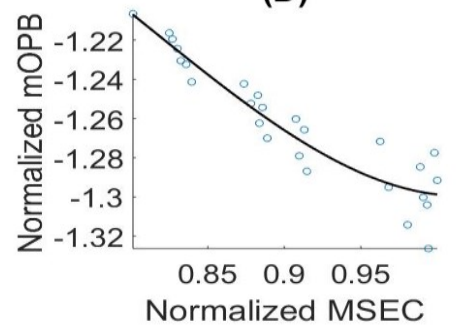

(B)

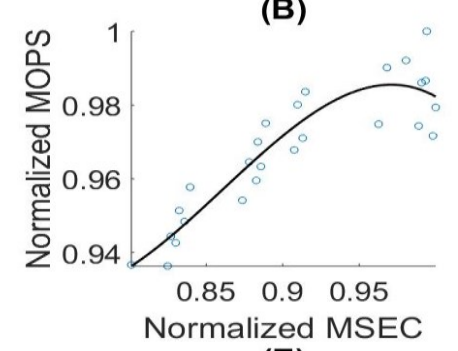

(E)

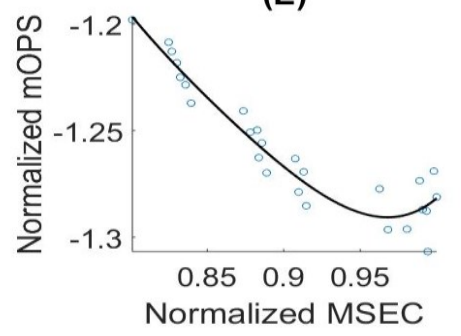

(C)

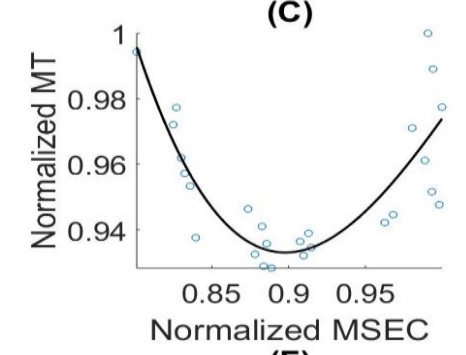

(F)

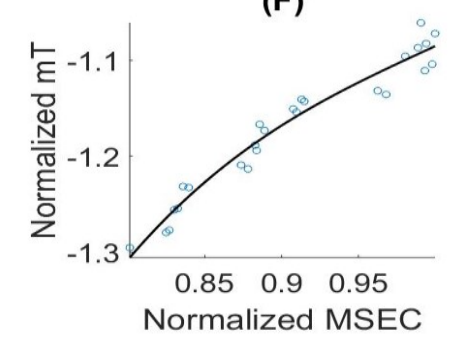

Figure 6.10. Normalized loads versus MSEC at $\bar{y}=0.25$. The scatter points are the correlated data and the solid lines are the polynomial fits for the following loads: A) Maximum out of plane bending moment (MOPB); B) Maximum out of plane shear force (MOPS); C) Maximum torsion moment (MT); D) Minimum out of plane bending moment (mOPB); E) Minimum out of plane shear force (mOPS); F) Minimum torsion moment (mT).

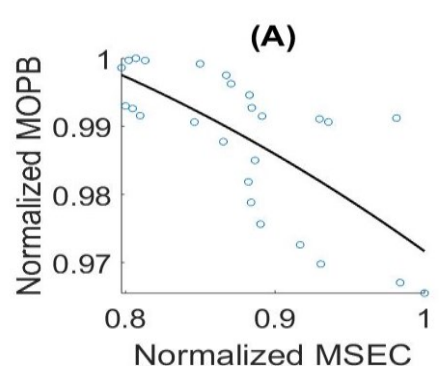

(D)

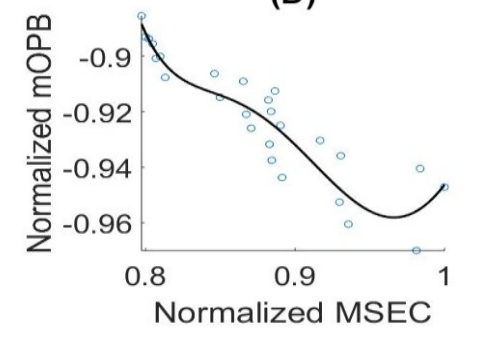

(B)

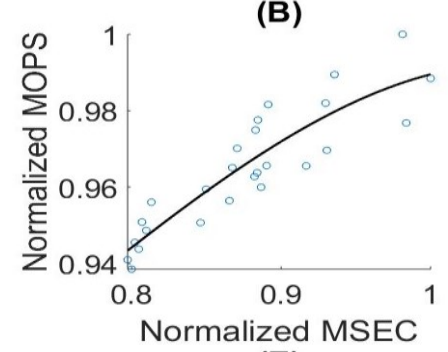

(E)

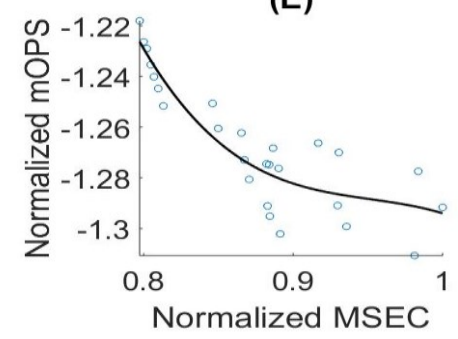

(C)

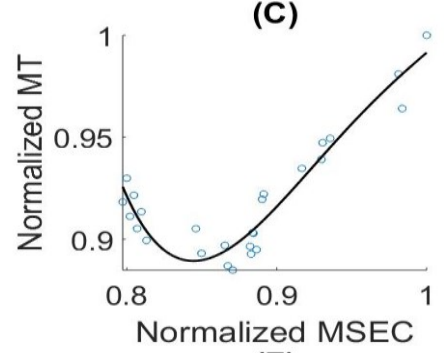

(F)

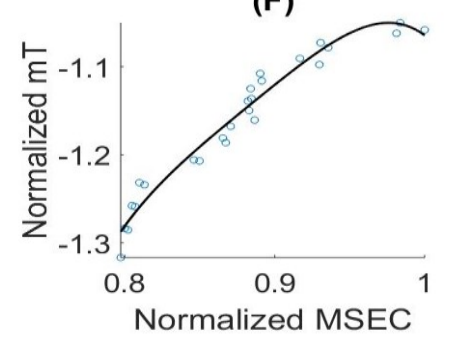

Figure 6.11. Normalized loads versus MSEC at $\bar{y}=0.50$. The scatter points are the correlated data and the solid lines are the polynomial fits for the following loads: A) Maximum out of plane bending moment (MOPB); B) Maximum out of plane shear force (MOPS); C) Maximum torsion moment (MT); D) Minimum out of plane bending moment (mOPB); E) Minimum out of plane shear force (mOPS); F) Minimum torsion moment (mT). 
(A)

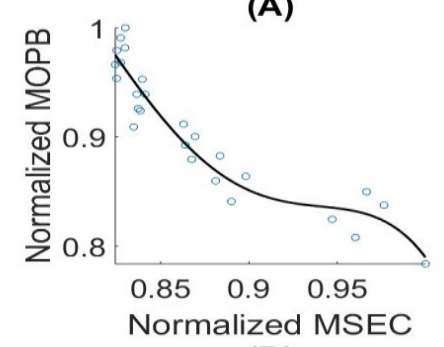

(D)

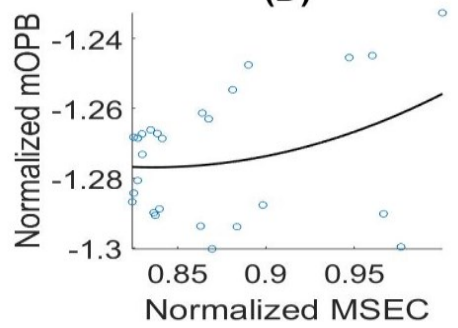

(B)

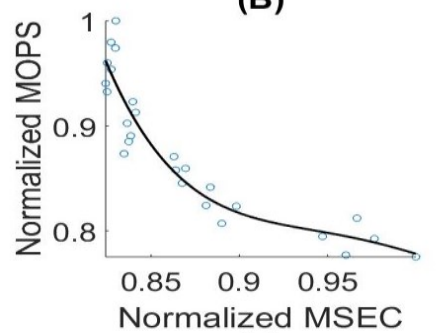

(E)

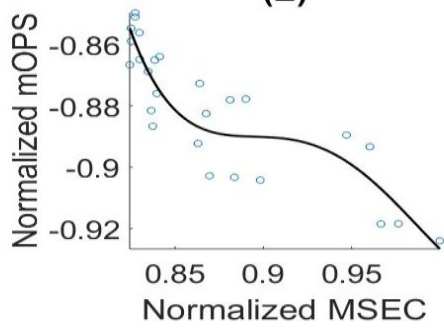

(C)

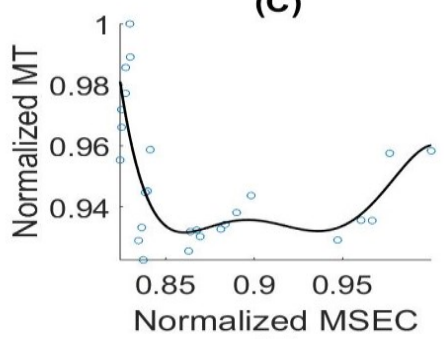

(F)

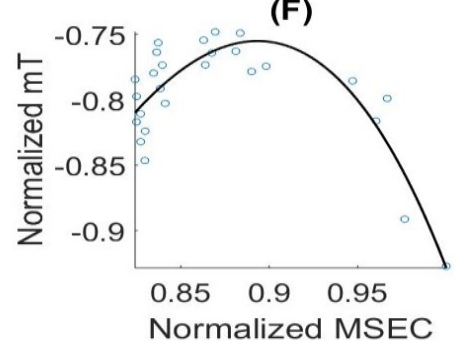

Figure 6.12. Normalized loads versus MSEC at $\bar{y}=0.75$. The scatter points are the correlated data and the solid lines are the polynomial fits for the following loads: A) Maximum out of plane bending moment (MOPB); B) Maximum out of plane shear force (MOPS); C) Maximum torsion moment (MT); D) Minimum out of plane bending moment (mOPB); E) Minimum out of plane shear force (mOPS); F) Minimum torsion moment (mT).

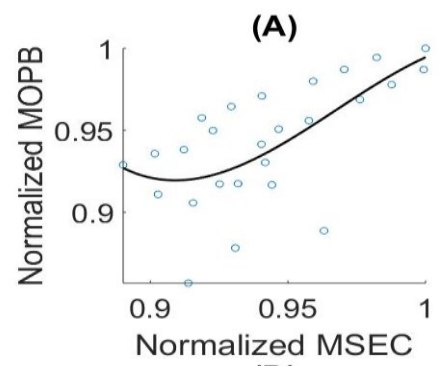

(D)

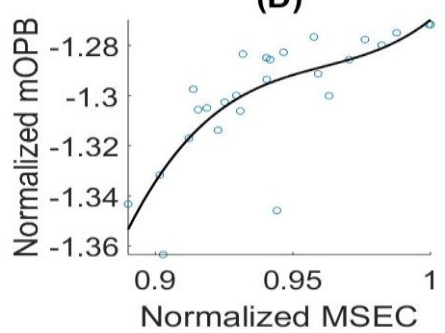

(B)

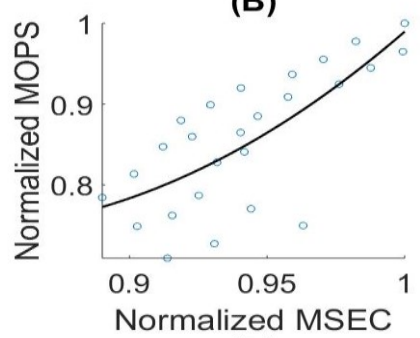

(E)

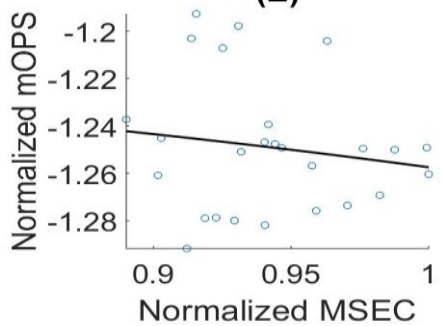

(C)

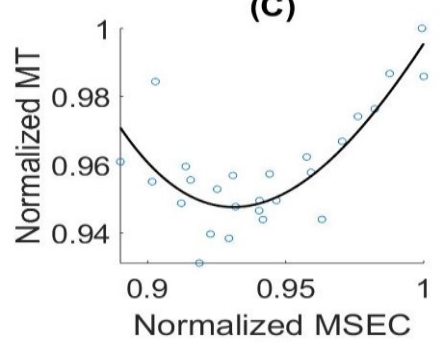

(F)

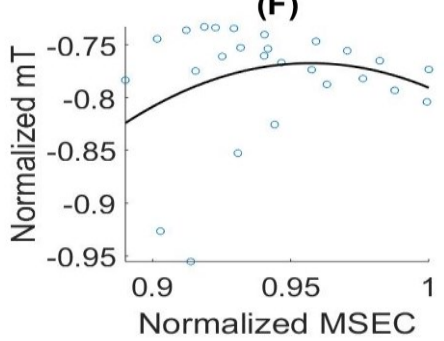

Figure 6.13. Normalized loads versus MSEC at the wing tip, $\bar{y}=1.0$. The scatter points are the correlated data and the solid lines are the polynomial fits for the following loads: A) Maximum out of plane bending moment (MOPB); B) Maximum out of plane shear force (MOPS); C) Maximum torsion moment (MT); D) Minimum out of plane bending moment (mOPB); E) Minimum out of plane shear force (mOPS); F) Minimum torsion moment (mT). 


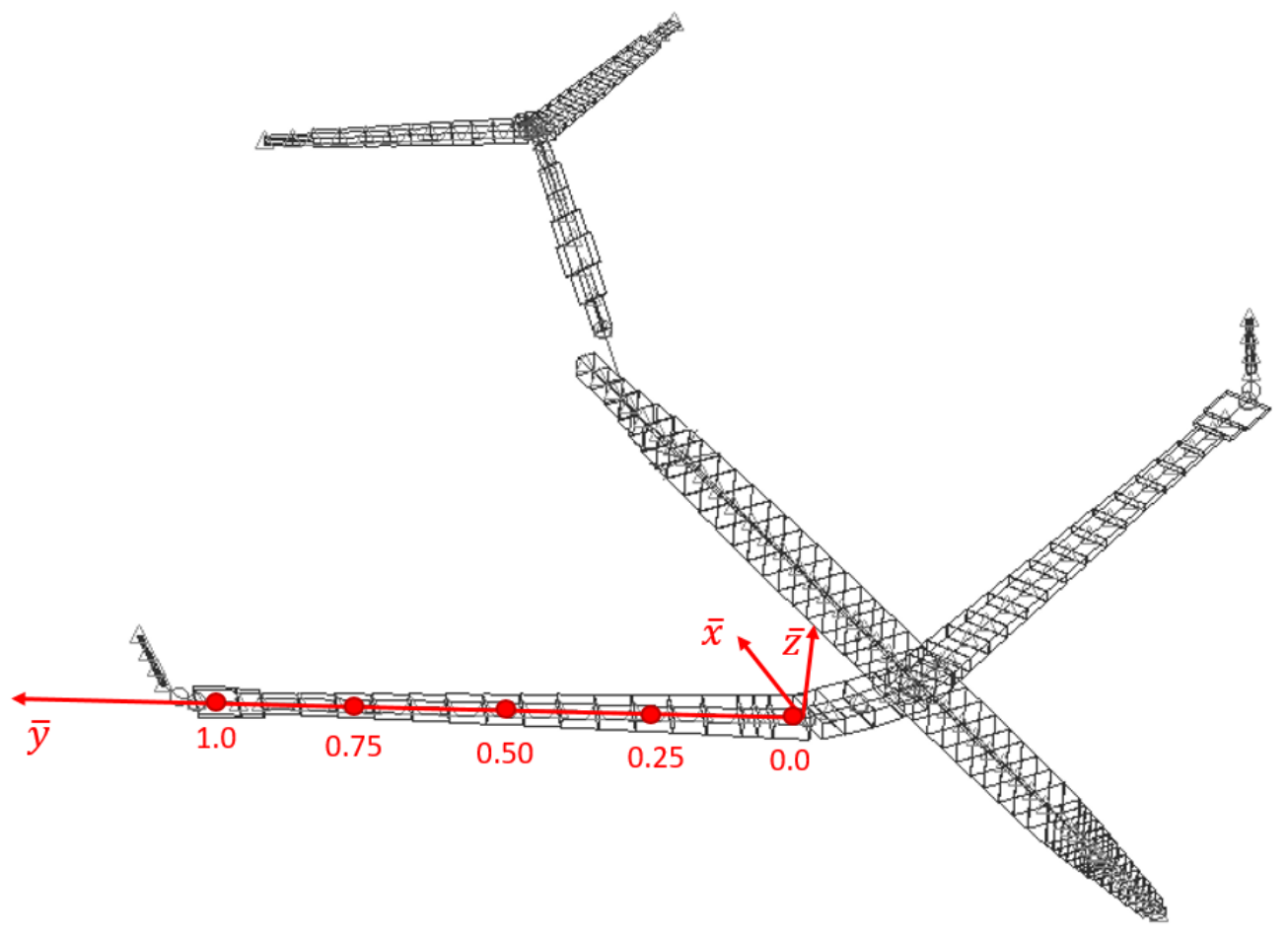

Figure 6.14. Monitored elements and its normalized location with respect to the wing root location.

The design space point selected to perform the dummy optimization is located at an altitude of $29500 \mathrm{ft}$. and a Mach number of 0.83 . This point was selected randomly, since prior to the design exploration no assumptions can me made as to what point could draw more or less information about the behaviour of the design space during optimization.

The behaviour of each load case with respect to the variation of MSEC differs from one another and it becomes apparent that certain loads are more sensitive than others. This is even more apparent in Table 6.2, where the combination of elements and loads that triggered the reexploration of the design space (if any) is highlighted. There, the estimated incremental loads were normalized with respect to its threshold $\gamma$ (in fact, each load type possesses its own threshold, 
since the orders of magnitude are different), so any number above 1 indicates that the threshold was surpassed.

\begin{tabular}{|c|c|c|c|c|c|}
\hline iteration & $\bar{y}$ & load code & $\max \overline{\Delta L}$ & $\min \overline{\Delta L}$ & $\gamma$ \\
\hline \multirow{15}{*}{2} & 0.00 & OPB & 0.34 & 0.72 & $<$ \\
\hline & 0.00 & OPS & 0.06 & 0.07 & $<$ \\
\hline & 0.00 & $\mathrm{~T}$ & 0.05 & 0.21 & $<$ \\
\hline & 0.25 & OPB & 0.02 & 0.44 & $<$ \\
\hline & 0.25 & OPS & 0.02 & 0.04 & $<$ \\
\hline & 0.25 & $\mathrm{~T}$ & 0.21 & 0.26 & $<$ \\
\hline & 0.50 & OPB & 0.09 & 0.59 & $<$ \\
\hline & 0.50 & OPS & 0.03 & 0.02 & $<$ \\
\hline & 0.50 & $\mathrm{~T}$ & 0.15 & 0.20 & $<$ \\
\hline & 0.75 & OPB & 0.03 & 0.01 & $<$ \\
\hline & 0.75 & OPS & 0.01 & 0.00 & $<$ \\
\hline & 0.75 & $\mathrm{~T}$ & 0.00 & 0.02 & $<$ \\
\hline & 1.00 & OPB & 0.02 & 0.01 & $<$ \\
\hline & 1.00 & OPS & 0.01 & 0.00 & $<$ \\
\hline & 1.00 & $\mathrm{~T}$ & 0.00 & 0.01 & $<$ \\
\hline 3 & 0.00 & OPB & 0.74 & 1.55 & $>$ \\
\hline \multirow{7}{*}{4} & 0.00 & OPB & 0.39 & 0.82 & $<$ \\
\hline & 0.00 & OPS & 0.05 & 0.08 & $<$ \\
\hline & 0.00 & $\mathrm{~T}$ & 0.02 & 0.23 & $<$ \\
\hline & 0.25 & OPB & 0.10 & 0.22 & $<$ \\
\hline & 0.25 & OPS & 0.01 & 0.02 & $<$ \\
\hline & 0.25 & $\mathrm{~T}$ & 0.01 & 0.05 & $<$ \\
\hline & 0.50 & OPB & 0.00 & 0.04 & $<$ \\
\hline
\end{tabular}




\begin{tabular}{|c|c|c|c|c|c|}
\hline & 0.50 & OPS & 0.00 & 0.00 & $<$ \\
\hline & 0.50 & $\mathrm{~T}$ & 0.01 & 0.01 & $<$ \\
\hline & 0.75 & OPB & 0.09 & 0.01 & $<$ \\
\hline & 0.75 & OPS & 0.02 & 0.00 & $<$ \\
\hline & 0.75 & $\mathrm{~T}$ & 0.00 & 0.01 & $<$ \\
\hline & 1.00 & OPB & 0.03 & 0.01 & $<$ \\
\hline & 1.00 & OPS & 0.02 & 0.00 & $<$ \\
\hline & 1.00 & $\mathrm{~T}$ & 0.00 & 0.00 & $<$ \\
\hline 5 & 0.00 & OPB & 0.65 & 1.38 & $>$ \\
\hline \multirow{15}{*}{6} & 0.00 & OPB & 0.13 & 0.29 & $<$ \\
\hline & 0.00 & OPS & 0.01 & 0.02 & $<$ \\
\hline & 0.00 & $\mathrm{~T}$ & 0.01 & 0.08 & $<$ \\
\hline & 0.25 & OPB & 0.06 & 0.14 & $<$ \\
\hline & 0.25 & OPS & 0.01 & 0.01 & $<$ \\
\hline & 0.25 & $\mathrm{~T}$ & 0.00 & 0.03 & $<$ \\
\hline & 0.50 & OPB & 0.05 & 0.53 & $<$ \\
\hline & 0.50 & OPS & 0.03 & 0.02 & $<$ \\
\hline & 0.50 & $\mathrm{~T}$ & 0.08 & 0.16 & $<$ \\
\hline & 0.75 & OPB & 0.10 & 0.01 & $<$ \\
\hline & 0.75 & OPS & 0.05 & 0.02 & $<$ \\
\hline & 0.75 & $\mathrm{~T}$ & 0.07 & 0.02 & $<$ \\
\hline & 1.00 & OPB & 0.02 & 0.00 & $<$ \\
\hline & 1.00 & OPS & 0.01 & 0.00 & $<$ \\
\hline & 1.00 & $\mathrm{~T}$ & 0.00 & 0.00 & $<$ \\
\hline 7 & 0.00 & OPB & 0.61 & 1.36 & $>$ \\
\hline
\end{tabular}

To evaluate the quality of the interpolated surfaces, the MOPB evaluated at the wing root $(\bar{y}=$ 0.00) during the first iteration is used as the main example. This surface was reconstructed using 
$22 \%$ of the points that make up the design space (97 out of 435), using an Expected Improvement threshold (E) of 1. Figure 6.15, shows the true and the interpolated surfaces overlapped, where the black dots show the location of the observations. Figure 6.16 shows the diagnosis plot with the confidence interval lying between $[-1,+1]$. Finally, in Figure 6.17 the percentile error of the estimated surface is seen to be bounded between $[-5,+3]$. Excellent agreement is found between the true and the estimated surface response, but more importantly, since roughly $22 \%$ of the points are required to construct the full design space, a $78 \%$ reduction in time is achieved every time that a design space re-exploration is triggered.

For the remaining surfaces to be interpolated and through the following iterations, only some new points will be added to the sample response vector $\boldsymbol{Y}(\boldsymbol{X})$, if and only if, the Expected Improvement function is larger than the threshold (E). This can be seen more clearly in Table 6.3, where the normalized response surfaces of the 6 monitored loads for the first and the last iteration are shown for the first and the last elements, i.e. the wing $\operatorname{root}(\bar{y}=0.0)$ and the wing tip $(\bar{y}=1.0)$. 


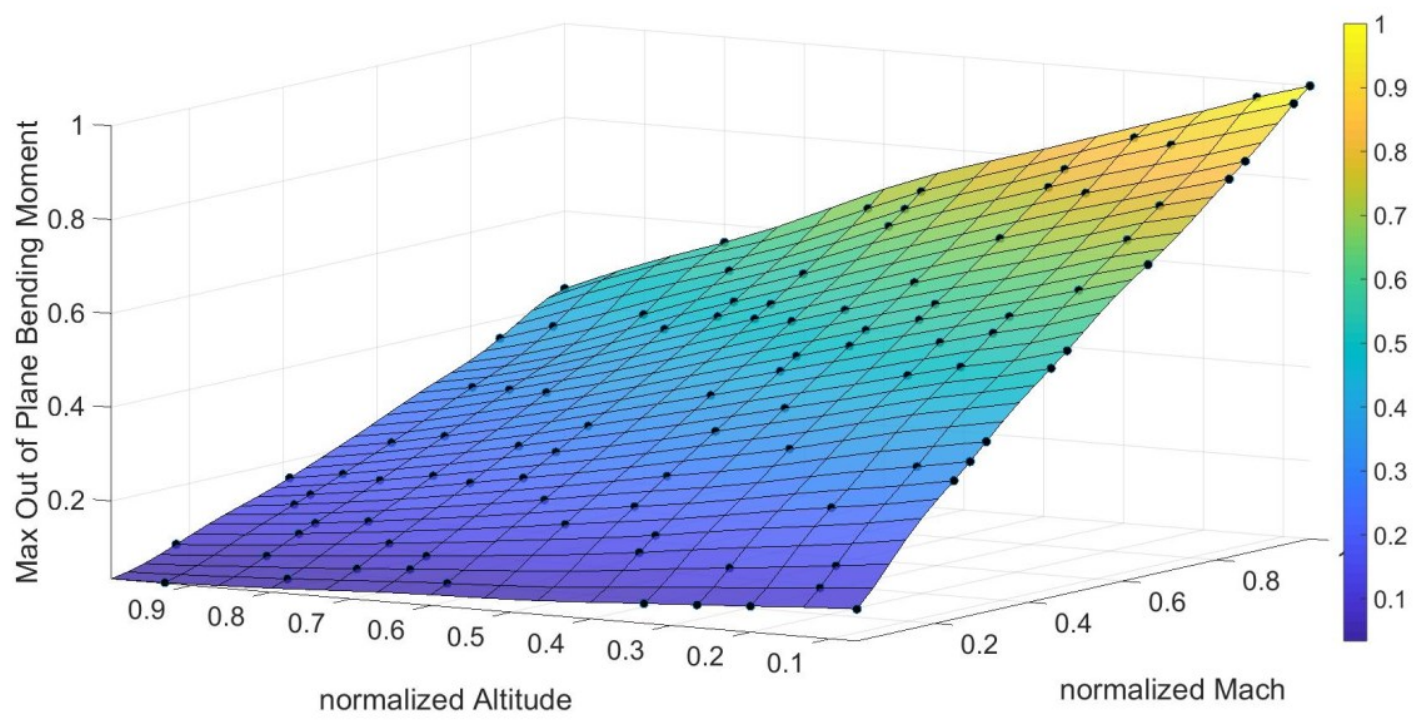

Figure 6.15. Normalized estimated max out of plane bending moment at the wing root $(\bar{y}=$ 0.00) during the first iteration as a function of Mach number and altitude with an Expected Improvement threshold of 1, with 97 sample points

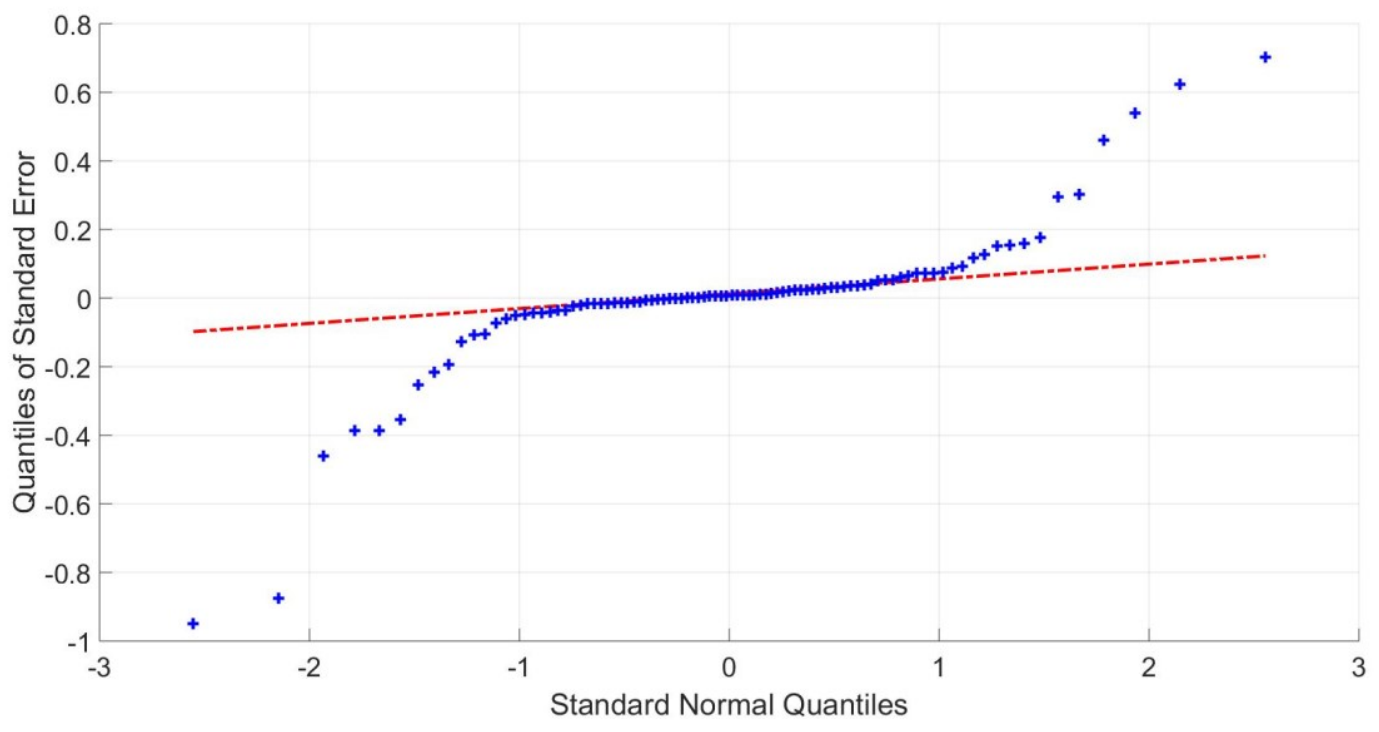

Figure 6.16. QQ plot of the max out of plane bending moment at the wing root with 84 initial sample points selected using the LH scheme. 


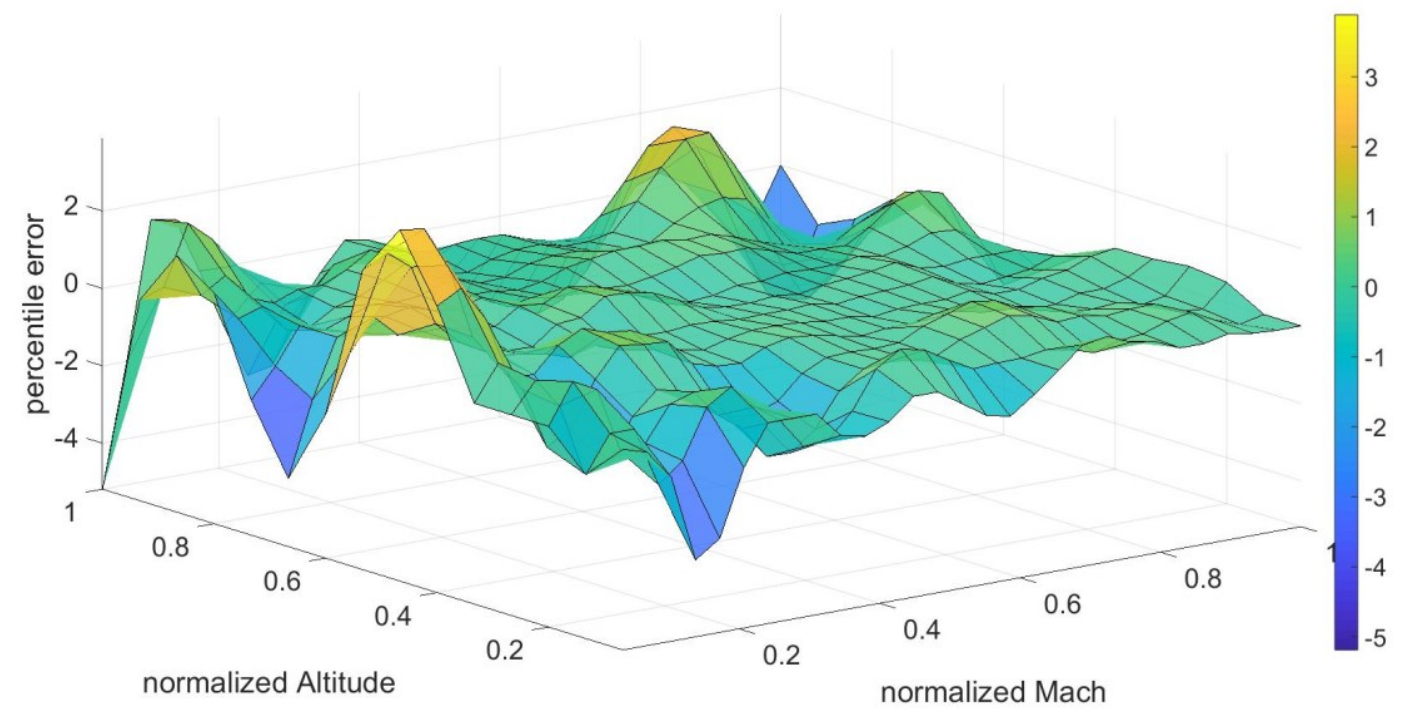

Figure 6.17. Percentile error of the interpolated max out of plane bending moment at the wing root as a function of Mach number and altitude with an Expected Improvement threshold of 1 .

Table 6.3. Normalized response surfaces of the 6 monitored loads for the first and the last iteration evaluated at the wing root $(\bar{y}=0.0)$ and the wing tip $(\bar{y}=1.0)$.

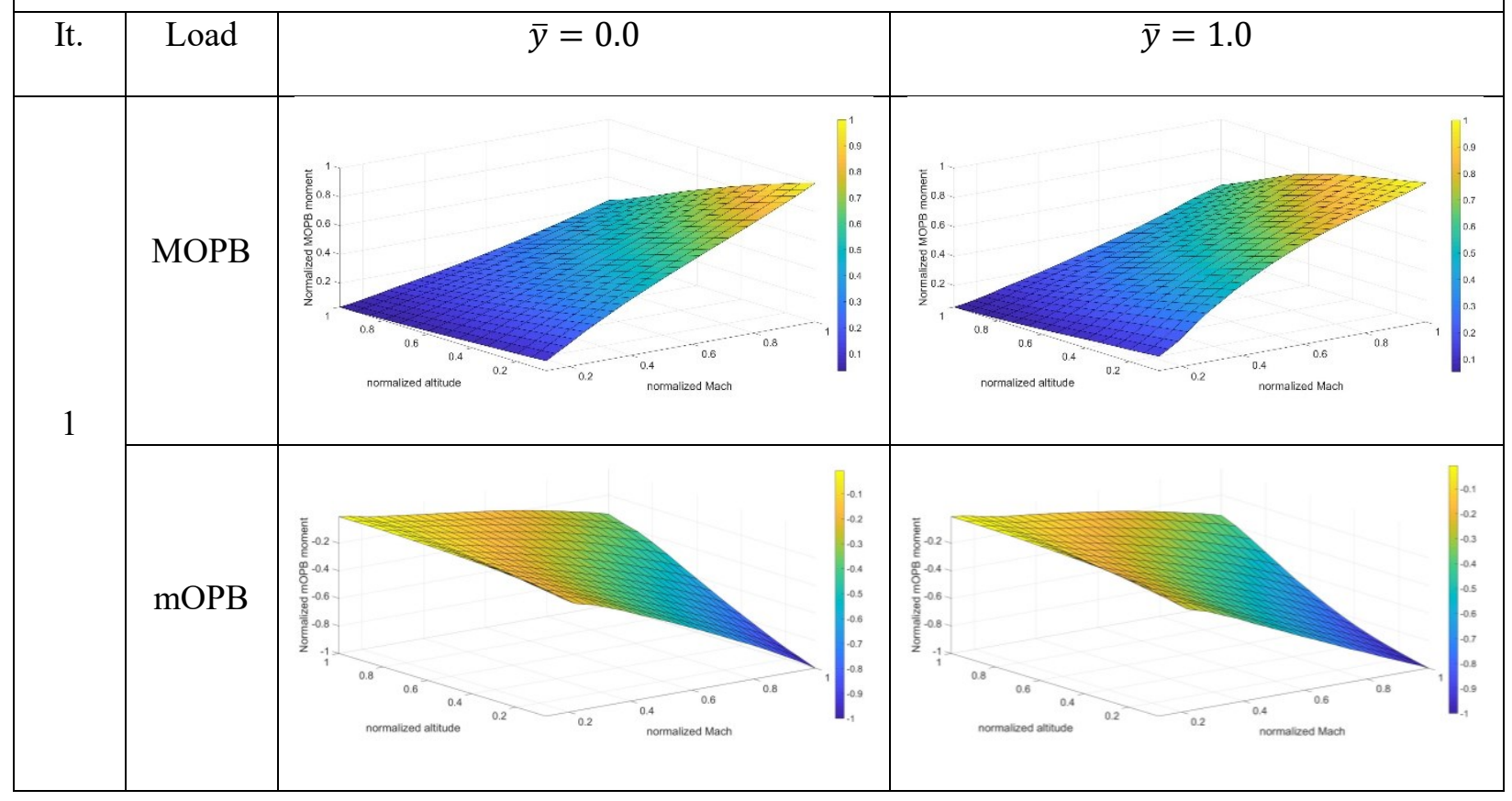




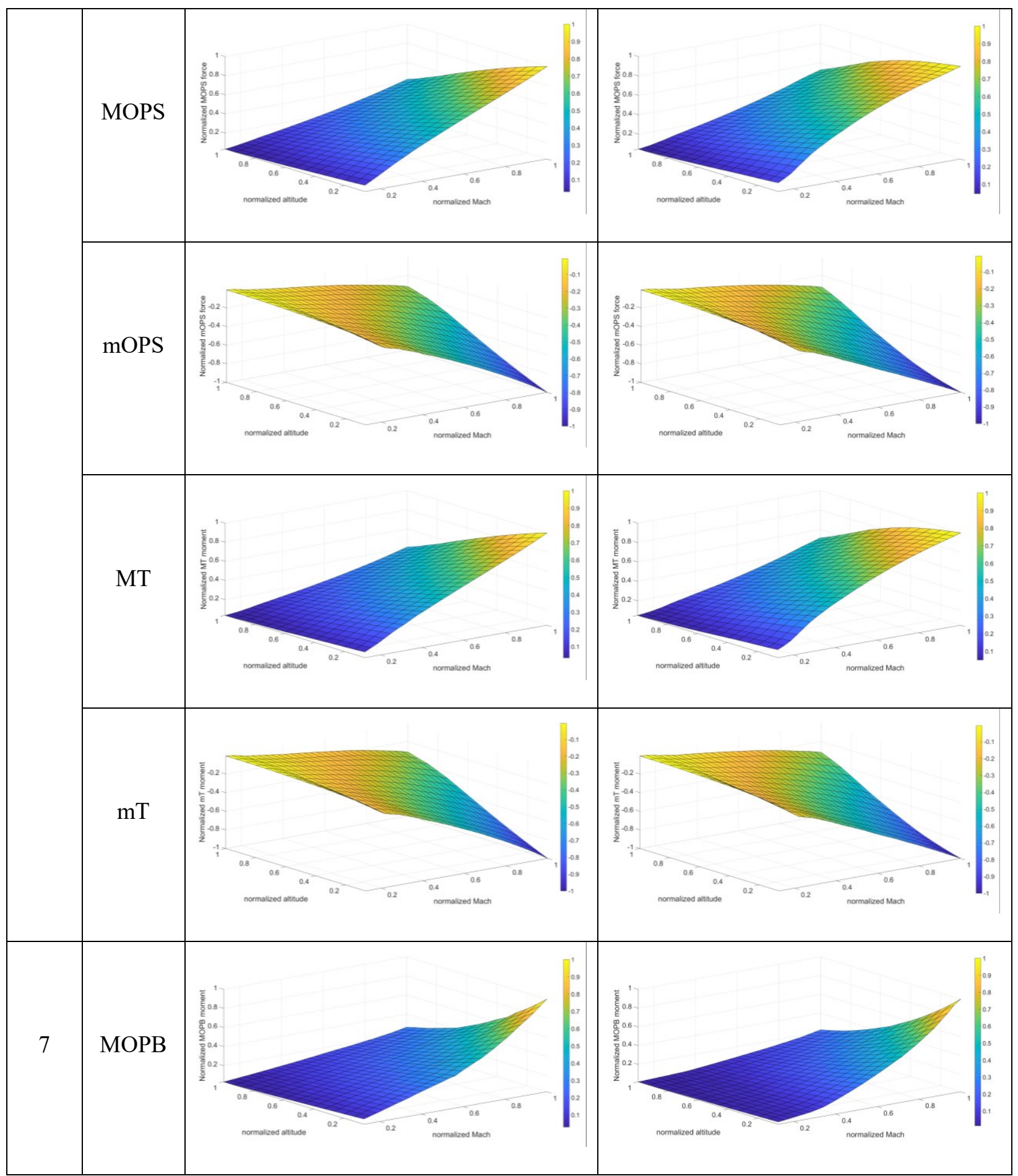




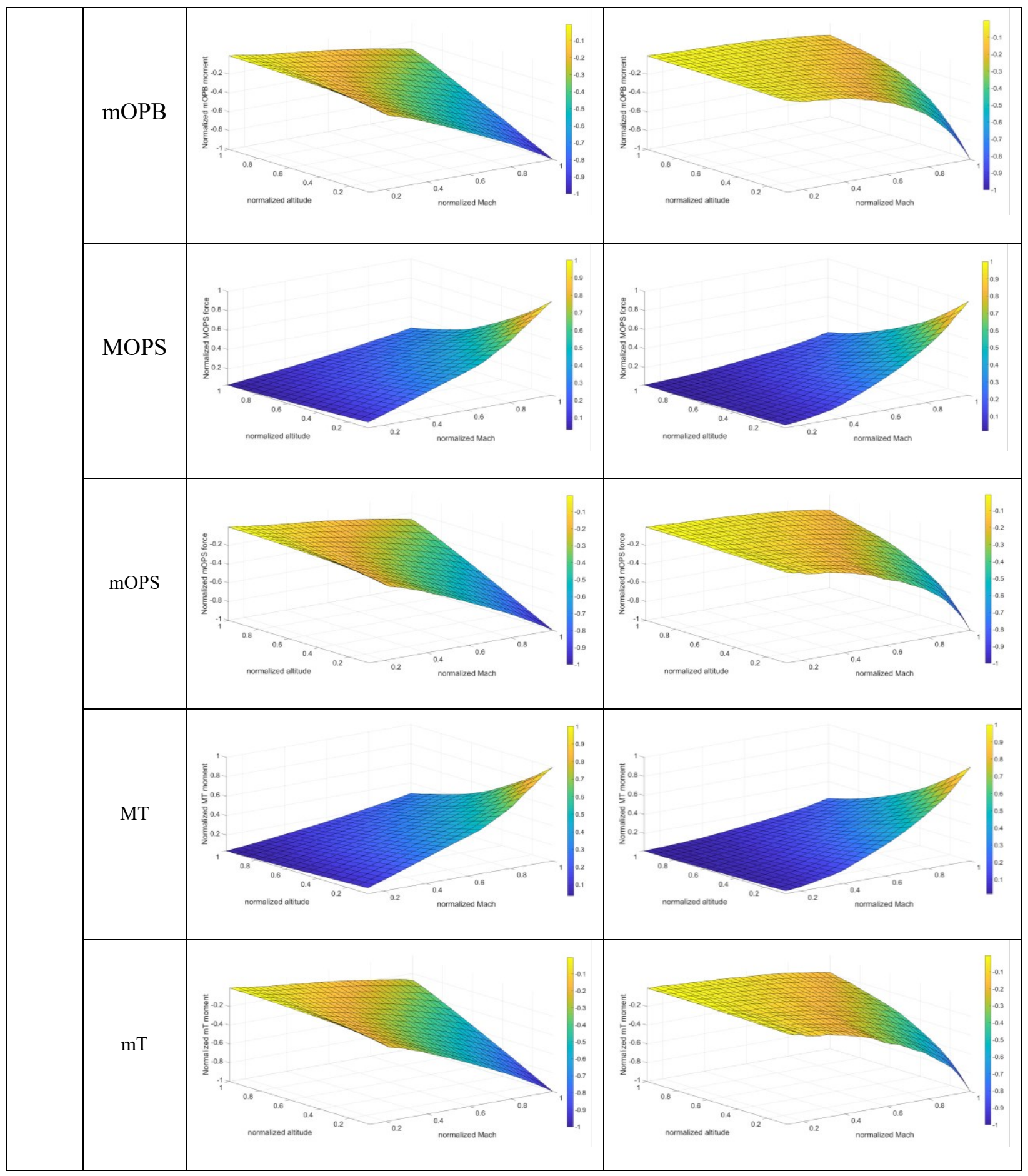

It is noticed that all the response surfaces in Table 6.3 possess one important characteristic: they are all unimodal. This provides a great advantage, since, very few new sample points are added 
when evaluating the different loads at different stations throughout the structural parametric variations. Thus, by exploiting the similarities between the families of curves, higher computational savings are achieved.

In the case of more complex response surfaces, such as a multimodal function, or where the family of surfaces to interpolate do not possess similar characteristics, one should expect lesser computational savings, since more data points will be automatically requested by the algorithm in order to provide a high quality interpolation.

From each response surface, the global maximum and global minimums are found. If these are part of the sample sites, then the program calls the result files obtained from Nastran, finds the gust case and time step at which they occur and extracts the time consistent loads (Figure 6.18, shows the variation of the normalized time consistent MOPB moment and the mOPB moment with respect to the initial exploration of the design space, i.e. iteration 1, extracted for the wing root element during the optimization process). Otherwise, the location of the $\min / \max$ is added to the sample sites, a new simulation is run, and the process described above repeated.

This last step provides as an additional advantage. The location of the current incremental $\mathrm{min} / \mathrm{max}$ will always be added to the sample vector, providing a zero error between the true and estimated incremental $\mathrm{min} / \mathrm{max}$ response. This can be seen in Figure 6.19 and Figure 6.20, where the estimated and the true MOPB and $\mathrm{mOPB}$ are shown as a function of the iteration number. 


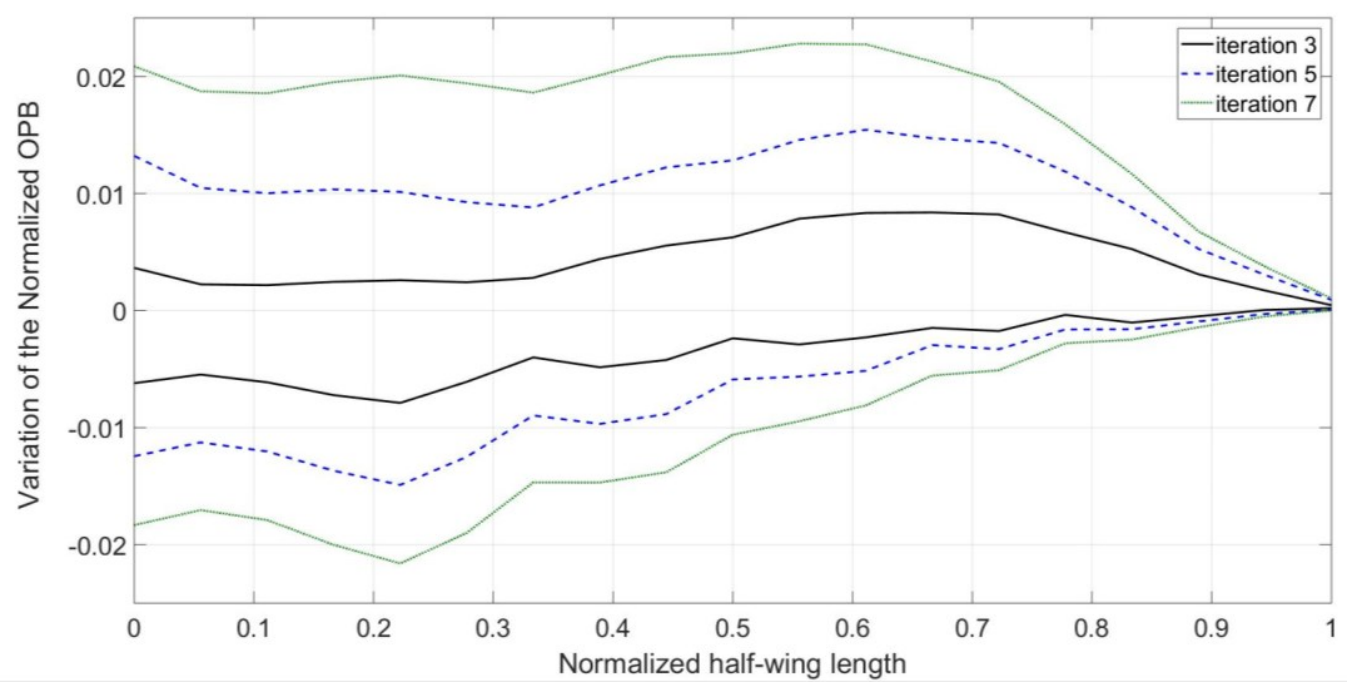

Figure 6.18. Variation of the normalized out of plane bending moment of the time consistent loads as a function of normalized half-wing length.

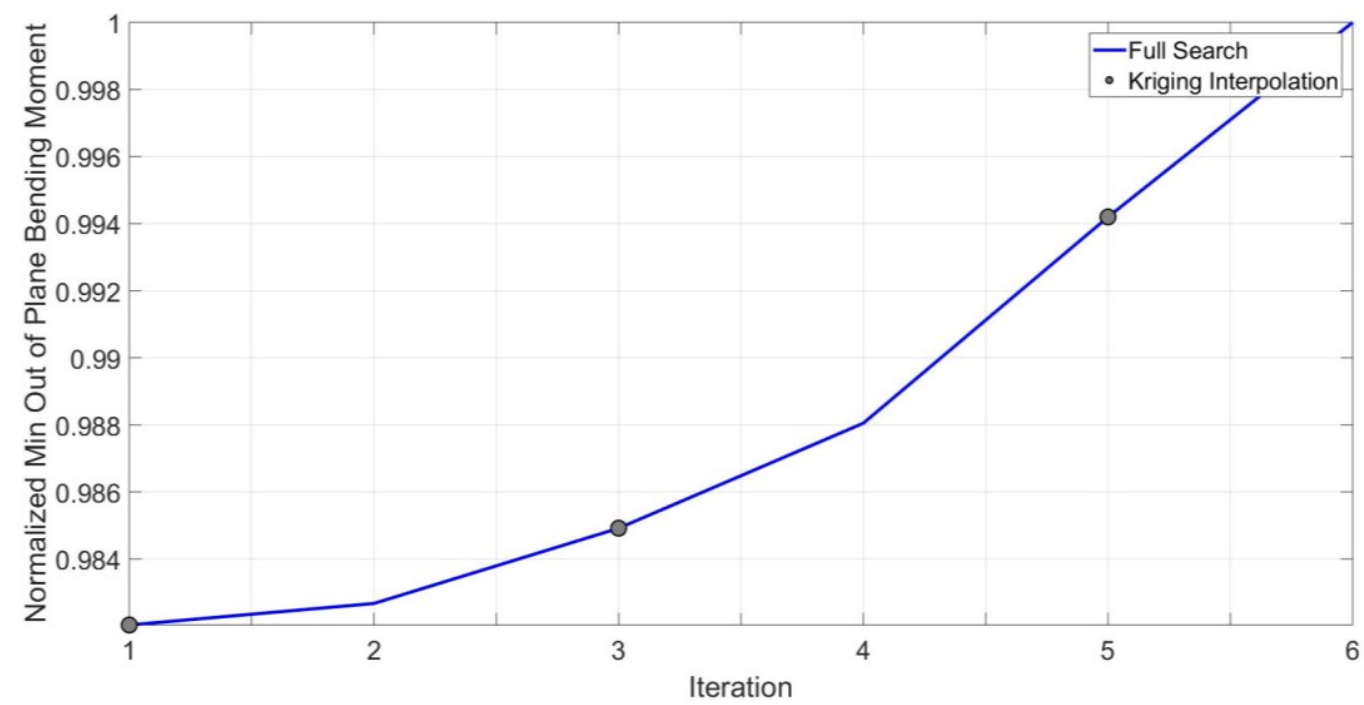

Figure 6.19. Maximum out of plane bending moment (MOPB) at the wing $\operatorname{root}(\bar{y}=0.0)$ as a function of the iteration number. 


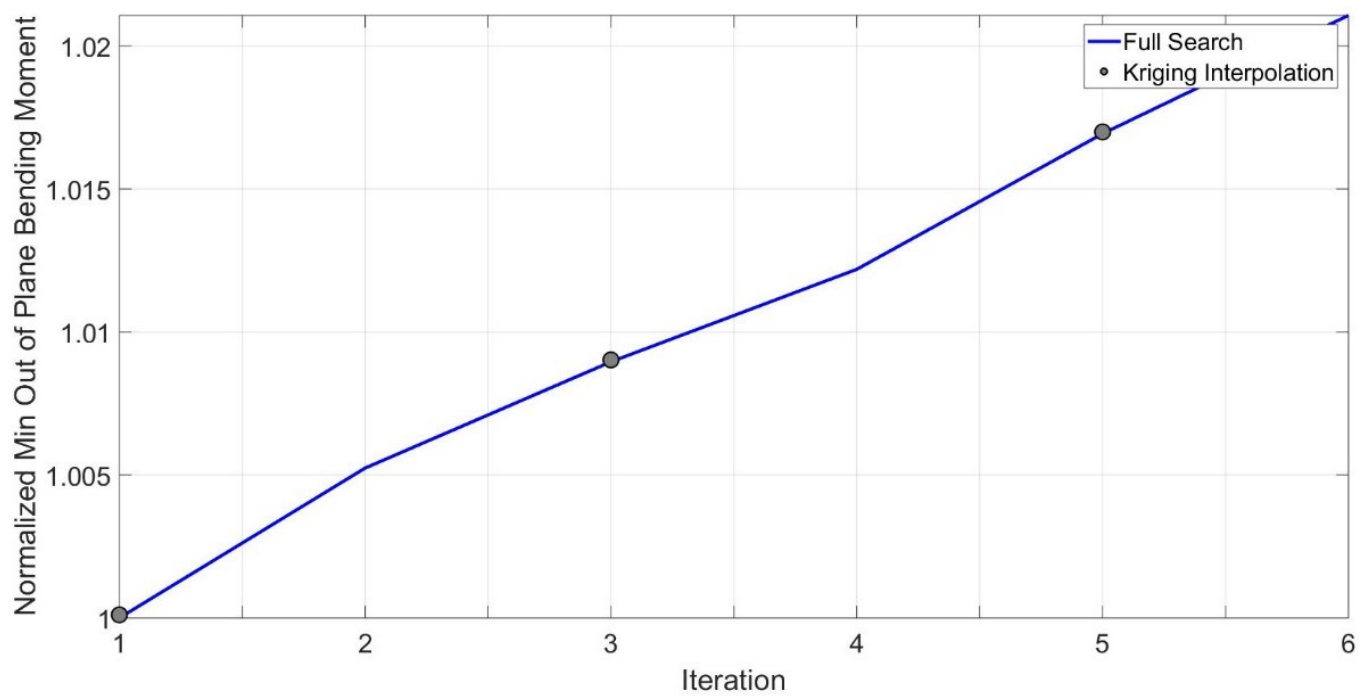

Figure 6.20. Minimum out of plane bending moment (mOPB) at the wing $\operatorname{root}(\bar{y}=0.0)$ as a function of the iteration number.

\subsubsection{Summary of results}

To finalize, the performance of the full factorial search vs the proposed methodology is compared in Table 6.4. The simulations were performed on a workstation with a Quad-core $4.20 \mathrm{GHz}$ processor, where a single processor was used. A total time reduction of $84 \%$ was achieved.

\begin{tabular}{|c|c|c|c|c|}
\hline \multicolumn{2}{|c|}{$\begin{array}{c}\text { Table 6.4. Comparison of the performance between the full factorial search and the proposed } \\
\text { methodology. The * indicates that n simulations where run whenever the MSEC trigger was } \\
\text { surpassed. }\end{array}$} \\
\hline Analysis & $\begin{array}{c}\text { Total time of } \\
\text { execution [hrs] }\end{array}$ & \# of iterations & $\begin{array}{c}\text { \# of simulation runs per } \\
\text { iteration }\end{array}$ & $\begin{array}{c}\text { Time to construct the } \\
30 \text { response surfaces } \\
{[\mathrm{hrs}]}\end{array}$ \\
\hline $\begin{array}{c}\text { Full Factorial } \\
\text { Search }\end{array}$ & 25.375 & 7 & 435 & 3.625 \\
\hline EGO + MSEC & 3.440 & 7 & $97 *$ & 0.800 \\
\hline
\end{tabular}


A summary of the mean percentage error between the interpolated and the true surfaces for the first iteration is presented in Table 6.5, where very good agreement if find.

\begin{tabular}{|c|c|c|c|c|c|c|}
\hline \multicolumn{6}{|c|}{ Table 6.5. Mean percentage error of the interpolated surfaces during the first iteration. } \\
\hline Element & $\begin{array}{c}\text { MOPB } \\
\text { moment }\end{array}$ & $\begin{array}{c}\text { mOPB } \\
\text { moment }\end{array}$ & $\begin{array}{c}\text { MOPS } \\
\text { force }\end{array}$ & $\begin{array}{c}\text { mOPS } \\
\text { force }\end{array}$ & $\begin{array}{c}\text { MT } \\
\text { moment }\end{array}$ & $\begin{array}{c}\text { mT } \\
\text { moment }\end{array}$ \\
\hline $\bar{y}=0.0$ & 0.1084 & 0.1052 & 0.0994 & 0.0874 & 0.3125 & 0.3155 \\
\hline $\bar{y}=0.25$ & 0.1010 & 0.1211 & 0.1217 & 0.1237 & 0.4372 & 0.4262 \\
\hline $\bar{y}=0.50$ & 0.0814 & 0.0713 & 0.1771 & 0.1872 & 0.4023 & 0.3022 \\
\hline $\bar{y}=0.75$ & 0.0326 & 0.0425 & 0.0554 & 0.0653 & 0.0216 & 0.0725 \\
\hline $\bar{y}=1.0$ & 0.0734 & 0.0733 & 0.0216 & 0.0315 & 0.3565 & 0.4575 \\
\hline
\end{tabular}

The error between the minimum/maximum loads recovered using the Surrogate-aided loads algorithm and the traditional full factorial search is zero for all loads and all elements that were monitored. This is because, as seen in Figure 6.19 and Figure 6.20, the algorithm selects the location of the minimum/maximum loads, adds the point as part of the sample vector, and runs the numerical simulation at that point. Thus, ensuring that the minimum/maximum points are computed and not estimated by the algorithm. 


\section{CONCLUSIONS AND FURTHER WORK}

Excellent agreement is found between the true and the estimated surface responses and a total time reduction of over $84 \%$ was achieved as shown by the case study. The time reduction is strongly dependent on the number of sample points required to provide a good fit. In this scenario, the response surface is simple enough that only a roughly $20 \%$ of the design space needs to be sampled. However, more complex responses will yield lesser computational savings due to the increased number of observations required to achieve the same level of accuracy.

Though the methodology is by no means restricted to simple functions, the user should keep in mind several considerations. For instance, in the case of multimodal functions, the magnitude and location of the global min/max may be obscured by the quality of the interpolation and the resolution of the grid size.

When the user possesses limited information about the response surface, the cross-validation test can provide an invaluable tool to assess the quality of the interpolation. It indicates the level of normality of the residuals, i.e. it indicates whether the difference between the magnitude of the known sample point and the magnitude of the kriging estimation, if the point is not part of the sample vector, has a normal probability distribution. It is also found that functions with normally behaving residuals will provide more accurate interpolations with fewer sample points.

When dealing with families of surfaces, it is desirable to exploit their similarities in order to minimize the number of observations required to provide a good fit. Otherwise, one might end up sampling too many points and no significant time reduction will occur. Finally, convex and affine 
functions, as in the present case, provide the highest computational savings and are best suited for the methodology presented herein.

From a practical application perspective, care should be taken in the selection of the design space, as pointed out by Khodaparast, H.H. \& Cooper, J.E. [54], if several response surfaces can be fully extracted from a single simulation, (such as the out of plane bending moment envelope, shown in Figure 5.3 as well as the out of plane shear force envelope and the twisting moment envelopes). Then the time to construct their surrogates may turn out to be the same or even higher. This, however, depends on to the judgment of the final user.

With respect to the correlation model, a more reliable prediction of the change in load, but still cheap to evaluate, would be preferable. The MSEC correlation is model and optimization dependent, i.e., the behaviour of the loads observed for this case study will not be valid for different kinds of structural optimization.

In general, different types of optimization will produce different variations in the overall mass and stiffness distribution, which will impact the final output. The shape of the design space will not necessarily change drastically, however, the rate at which it varies can be considerably different. This means that the polynomials used for this case study, cannot be used for any other model/optimization combination. If a new case study were to be developed, then a new set of polynomials would be required.

The Surrogate-Aided Response algorithm is powerful enough to be used during the development and the pre-sizing of structural components, specially when there is a need to investigate the behaviour of their response to parametric changes. On the other hand, if this method is to be used for certification purposes, it would be preferable to add a higher number of sample points (or 
increase the resolution of the grid), to ensure that the peak values (maximums and minimums) are properly captured.

\subsection{FURTHER WORK}

A potential candidate to replace the correlation model is the static modal participation factors (SMPF), as seen in Chapter 4, this method is tightly bounded by modal analysis and allows the estimation of the peak modal response without solving any differential equations. However, the equations of motions must be necessarily uncoupled.

Models with structural damping, non-linear modes, and added terms to classical equations of motion for MDoF systems (as the case is for aeroelastic models), do not yield to uncoupled equations after the modal transformation. Therefore, a different approach must be considered.

In the case of aeroelastic models, the aerodynamic influence coefficients (AIC's) act as external sources of stiffness and damping, which are dependent on the reduced frequency $(k)$ and the Mach number. An interesting idea to uncouple the equations of motion is to create a characteristic equation at each point in the sky. The added stiffness due to the AIC's augments to the structural stiffness matrix and by neglecting all types of damping, results in a new characteristic equation.

This would provide a more reliable and accurate manner to estimate when the change in the monitored load has exceeded a threshold at any point in the sample space. In fact, after the peak $\max /$ min values are located during the first iteration, the selected points in the design space would be that of the $\max / \mathrm{min}$ responses. In which case, higher computational saving may be attained. 


\section{APPENDIX I: SURROGATE-AIDED LOADS ALGORITHM}

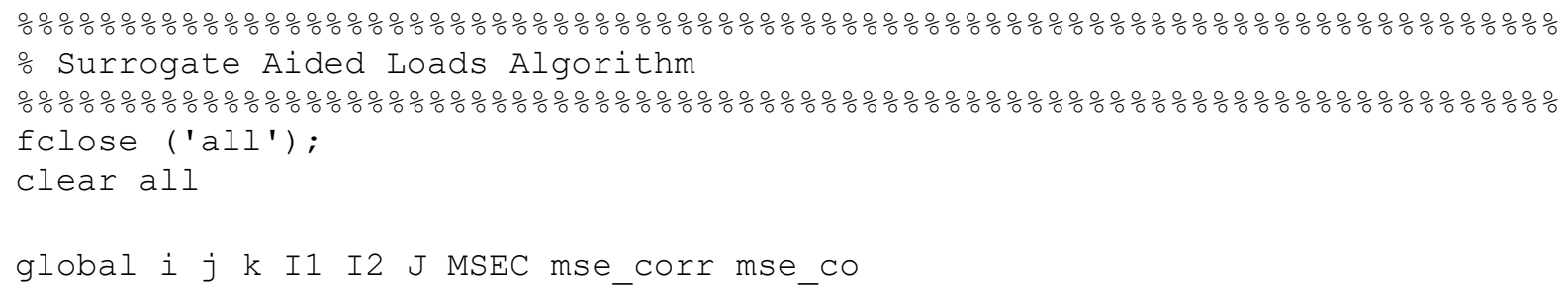


ㄴ Latin hypercube (0 to 1 )

$\mathrm{s}=2$;

$\mathrm{S}=\operatorname{lh} \operatorname{samp}\left(\mathrm{samp} \_\mathrm{p}, \mathrm{s}\right)$;

\% Adjust sampling points to ds

$\mathrm{S}(:, 1)=$ round $($ length $($ mach $) * \mathrm{~S}(:, 1))$;

$\mathrm{S}(:, 2)=$ round $($ length $($ alt $) * S(:, 2), 0)$;

ofliminate zeros from the sample sites

$\mathrm{S}(\mathrm{S}==0)=1$;

$S=$ unique $(S(:, 1: S)$, 'rows');

oUnobserved design sites

size_alt $=$ length (alt);

size_mach = length (mach);

$\mathrm{m}=1$;

for $j=1: 1:$ size_mach

for $i=1: 1:$ size_alt

$\mathrm{X}(\mathrm{m}, 1)=j ; \quad$ o columns (x axis)

$\mathrm{X}(\mathrm{m}, 2)=\mathrm{i} ; \quad$ orows $\quad(\mathrm{y}$ axis $)$

$\mathrm{m}=\mathrm{m}+1$;

end

end

응 SUBROUTINE 4 - VARIATIONS OF M\&K ("OPTIMIZER")

tic

$\mathrm{k}=0$;

for $i=1: 1:$ length (scaleB)

for $j=1: 1:$ length $(\mathrm{scaleH})$

$\mathrm{k}=\mathrm{k}+1$

ocompute new M\&K

[stiff2,massesl]=new_mk(base_file, mass_file, scaleB, scaleH, eqn);

oWrite new data - stiffness file

newpath = [folderp, '\wing_stiffness_case_', num2str(k), '.dat'];

fileID = fopen (newpath, 'w');

for $1=1$ : numel (stiff2)

end

fprintf(fileId, '⿳s $\left.\backslash n^{\prime}, \operatorname{stiff} 2\{1\}\right)$;

fclose (fileID);

\%Write new data - mass file

newpath $=$ [folderp, '\wing_mass_case_', num2str $(k)$, '. conm2'] ; 


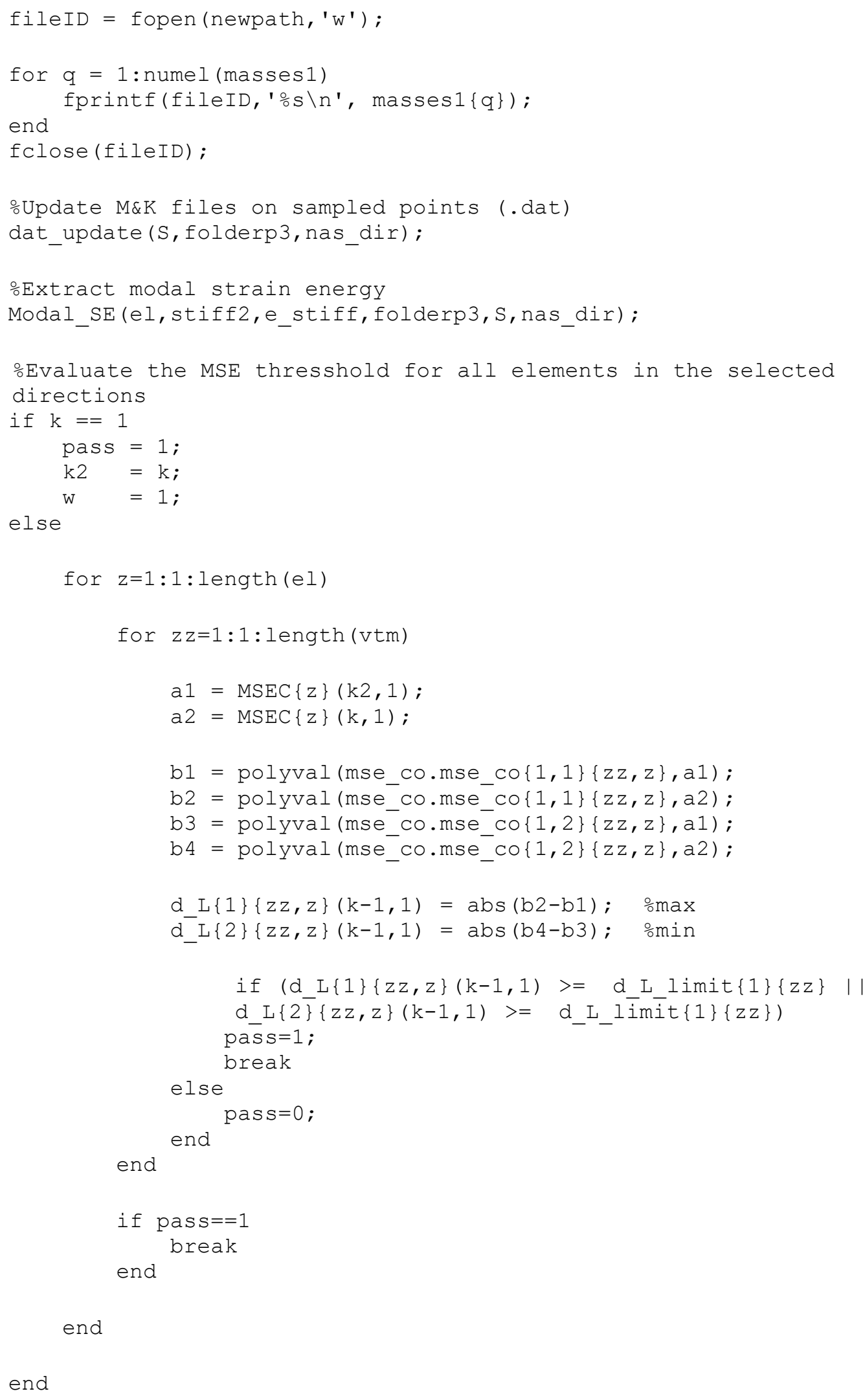


oIf no element/direction broke the above loop, then interpolate using the Kriging, olse re-evaluate MSE at a new M\&K distribution

if $\operatorname{pass}==0$

continue

else

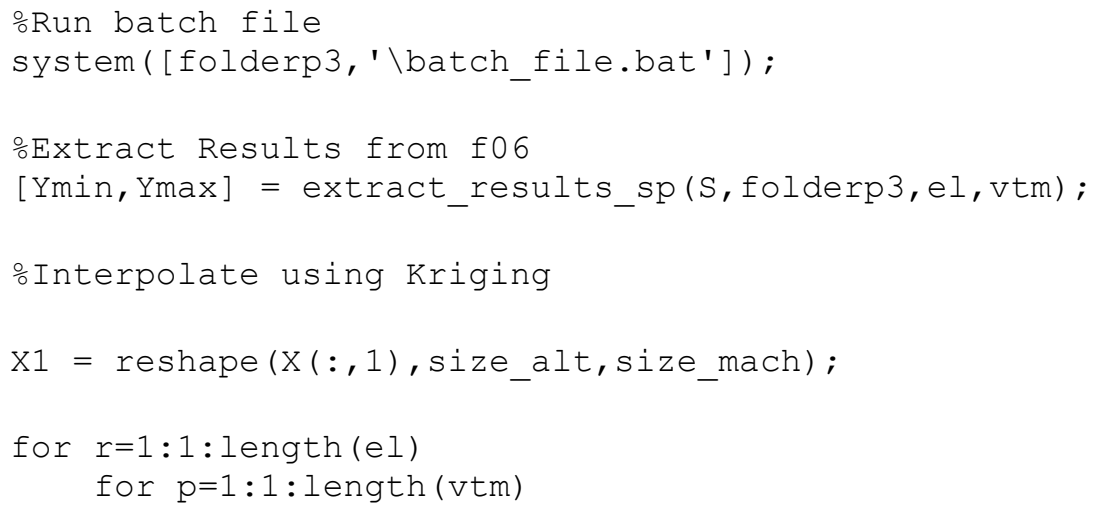




\section{REFERENCES}

[1] Bhatia, K. G., \& Company, T. B. (2001). Airplane Aeroelasticity: Practice and Potential. AIAA, (January).

[2] Cooper, J. E. (2014). From blue skies to green skies: how structural dynamics and uncertainty quantification can benefit future aircraft designs. Proceedings of the International Conference on Noise and Vibration Engineering ISMA 2014, 1-15.

[3] Hoblit, M. (1988). Gust Loads on Aircraft: Concepts and Applications. AIAA Education Series.

[4] “CS-25/EASA Certification Specifications for Large Aeroplanes". European Aviation Safety Agency. Amendment 3, September 2007.

[5] "FAR-25/FAA Certification Specifications for Transport Category Aircraft". Federal Aviation Administration, Washington, D.C., http://flightsimaviation.com/data/FARS/part_25-301.html [retrieved Dec. 2016]

[6] Wright, J. and J.E. Cooper. (2007) Introduction to Aircraft Aeroelasticity and Loads, McGraw Hill.

[7] Kier, T. M., Looye, G. H. N., Looye, G. H. N., Loads, G., Loads, M., \& Mechanics, F. (2009). Unifying manoeuvre and gust loads analysis models. International Forum of Aeroelasticity and Structural Dynamics (IFASD) 2009, 1-20. 
[8] Neubauer, M. \& Günther, G. (1999). Aging Aircraft Fleets: Structural and Other Subsystems Aspects. A. T. Defense Technical Information Center Compilation Part Notice.

[9] D’Vari, R., \& Baker, M. (1999). Aeroelastic loads and sensitivity analysis for structural loads optimization. Journal of Aircraft, 36(1), 156-166.

[10] Jones, D. R., Schonlau, M., \& Welch, W. J. (1998). Efficient global optimization of expensive black-box functions. Journal of Global optimization, 13(4), 455-492.

[11] Taylor, P., Hanson, L., \& Barnes, T. (2003). A Brief History of Aircraft Loads Analysis Methods. In 44th AIAA/ASME/ASCE/AHS/ASC Structures, Structural Dynamics, and Materials Conference (p. 1892).

[12] Flomenhoft, H. I., \& Gardens, P. B. (1994). Brief History of Gust Models for Aircraft Design, 31(5), 1225-1227.

[13] Lomax, T.L. (1996). Structural Loads analysis for commercial transport aircraft: Theory and Practice. AIAA Education Series.

[14] Vanderplaats, G. N., \& Problem, T. D. (1981). Structural Optimization—Past, Present, and Future. AIAA journal, 20(7), 992-1000.

[15] Bindolino, G., Ghiringhelli, G., Ricci, S. \& Terraneo, M. (2010). Multilevel Structural Optimization for Preliminary Wing-Box Weight Estimation. Journal of Aircraft. 47(2). 475489.

[16] Merchant, D. H., \& Sawdy, D. T. (1970). Monte Carlo Dynamic Analysis for Lunar Module Landing Loads, 8(1), 48-55. 
[17] Porter, R.F. \& Robinson A.C. (1971). A proposed criterion for aircraft flight in turbulence. National Technical Information Service, Springfield, Va. 22151.

[18] Jones, J. G. (1980). Modelling of gusts and wind shear for aircraft assessment and certification. Proceedings of the Indian Academy of Sciences Section C: Engineering Sciences, 3(1), 1-30.

[19] Stauffer, W. A., \& Hoblit, F. M. (1973). Dynamic Gust, Landing, and Taxi Loads Determinations in the Design of the \{L-1011\}. Journal of Aircraft, 10(8), 459-467.

[20] Bristow, J. W., \& Irving, P. E. (2007). Safety factors in civil aircraft design requirements. Engineering Failure Analysis.

[21] P. V. Thomas, M. S. A. ElSayed \& Denis Walch. (2018) "Review of Model Order Reduction Methods and their Applications in Aeroelasticity Loads Analysis for Design Optimization of Complex Airframes", ASCE, Journal of Aerospace Engineering.

[22] Haftka, R. T., \& Grandhi, R. V. (1986). Structural shape optimization-A survey. Computer Methods in Applied Mechanics and Engineering, 57(1), 91-106.

[23] Ding, Y. (1986). Shape optimization of structures: a literature survey. Computers \& Structures, 24(6), 985-1004.

[24] Grandhi, R. (1993). Structural Optimization with Frequency Constraints - A Review, 31(12), 2296-2303.

[25] Ting, T., Chen, T. L. C., \& Twomey, W. J. (1994). Automated Mode Tracking Strategy. AIAA Journal, 33(1), 183-185. 
[26] Kang, B. S., Park, G. J., \& Arora, J. S. (2006). A review of optimization of structures subjected to transient loads. Structural and Multidisciplinary Optimization, 31(2), 81-95.

[27] Rao, S. S. (1985). Optimization of airplane wing structures under gust loads. Computers and Structures, 21(4), 741-749.

[28] Rao, V. R., Iyengar, N. G. R., \& Rao, S. S. (1979). Optimization of wing structures to satisfy strength and frequency requirements. Computers and Structures, 10(4), 669-674.

[29] Choi, W. S., \& Park, G. J. (1999). Transformation of dynamic loads into equivalent static loads based on modal analysis. International Journal for Numerical Methods in Engineering, 46(1), 29-43.

[30] Jones, J. G. (1989). Statistical-Discrete-Dust Method for Predicting Aircraft Loads and Dynamic Response. Journal of Aircraft, 26(4), 382-392.

[31] Noback, R. (1986). Comparison of Discrete and Continuous Gust Methods for Airplane Design Loads Determination. Journal of Aircraft, v 23, n 3(3), 226-231.

[32] Fidkowski, K. J., Engelsen, F., Willcox, K. E., \& Kroo, I. M. (2008). Stochastic gust analysis techniques for aircraft conceptual design. 12th AIAA/ISSMO Multidisciplinary Analysis and Optimization Conference, (September), 1-16.

[33] Berci, M., Gaskell, P. H., Hewson, R. W., \& Toropov, V. V. (2013). A semi-analytical model for the combined aeroelastic behaviour and gust response of a flexible aerofoil. Journal of Fluids and Structures, 38, 3-21. 
[34] Pototzky, A. S., Perry, B. (iii), \& Zeiler, T. a. (1991). Calculating Time-correlated Gust Loads Using Matched Filter and Random Process Theories. Journal of Aircraft, 28(5), $346-352$.

[35] Scott, R. C., Potozky, A. S., \& Perry, B. (1993). Computation of maximized gust loads for nonlinear aircraft using matched-filter-based schemes. Journal of Aircraft, 30(5), 763768.

[36] Zeiler, T. a. (1997). Matched Filter Concept and Maximum Gust Loads. Journal of Aircraft, 34(1), 101-108.

[37] Vanderplaats, G. N. (1980). Comment on "Methods of Design Sensitivity Analysis in Structural Optimization.” AIAA Journal, 18(11), 1406-1407.

[38] Hsieh, C. C., \& Arora, J.S. (1984). Design Sensitivity Analysis and Optimization of Dynamic Response. Computer Methods in Applied Mechanics and Engineering, 43, 195219.

[39] Zole, A. (1994). Continuous Gust Response and Sensitivity Derivatives Using State-Space Models. Journal of Aircraft. 31(5), 1212-1214.

[40] Cerulli, C., Keulen, F. Van, Rixen, D. J., Introduction, I., \& Dynamics, E. (2007). Dynamic Reanalysis and Component Mode Synthesis to Improve Aircraft Modeling for Loads Calculation, (April), 1-15.

[41] Khodaparast, H. H., \& Cooper, J. E. (2014). Rapid Prediction of Worst-Case Gust Loads Following Structural Modification. AIAA Journal, 52(2), 242-254. 
[42] Uri, K. (2008). Reanalysis of Structures. AIAA Journal, 151(3), 504-511.

[43] Cacciola, P., Impollonia, N., \& Muscolino, G. (2005). A dynamic reanalysis technique for general structural modifications under deterministic or stochastic input. Computers and Structures, 83(14), 1076-1085.

[44] Noor, a. K., \& Lowders, H. E. (1974). Approximate techniques of structural reanalysis. Computers \& Structures, 4, 801-812.

[45] Kirsch, U. (2003). A unified reanalysis approach for structural analysis, design, and optimization. Structural and Multidisciplinary Optimization, 25(2), 67-85.

[46] Kirsch, U. (2010). Reanalysis and sensitivity reanalysis by combined approximations. Structural and Multidisciplinary Optimization, 40(1-6), 1-15.

[47] Akgün, M. A., Garcelon, J. H., \& Haftka, R. T. (2001). Fast exact linear and non-linear structural reanalysis and the Sherman-Morrison-Woodbury formulas. International Journal for Numerical Methods in Engineering, 50(7), 1587-1606.

[48] Abdulwahab, E. N., \& Hongquan, C. (2008). Aircraft gust load estimation due to atmospheric turbulence under different flight conditions. Aeronautical Journal, 112(1132), $345-352$.

[49] Knoblach, A. (2013). Robust Performance Analysis Applied to Gust Loads Computation, $3(1), 39-50$. 
[50] Gano, S. E., Kim, H., \& Ii, D. E. B. (2006). Comparison of Three Surrogate Modeling Techniques: Datascape, Kriging, and Second Order Regression. 11th AIAA/ISSMO Multidiscplinary Analysis and Optimization Conference, (September), 6-8.

[51] Martin, J. D., \& Simpson, T. W. (2005). Use of Kriging Models to Approximate Deterministic Computer Models. AIAA Journal 43(4) 853-863.

[52] Alonso, J.J., LeGresley, P., \& Pereyra, V. (2009). Aircraft design optimization. Mathematics and Computers in Simulation. 79. 1948-1958.

[53] Han, Z.-H., Görtz, S., \& Hain, R. (2010). A Variable-Fidelity Modeling Method for AeroLoads Prediction. Notes on Numerical Fluid Mechanics and Multidisciplinary Design, $112,17-25$.

[54] Khodaparast, H. H., \& Cooper, J. E. (2012). Efficient Worst Case "1-Cosine" Gust Loads Prediction. ASD Journal. 2(3), 33-54.

[55] Ghosh, S., Rancourt, D., \& Mavris, D. N. (2015). Principal Component Analysis Assisted Surrogate Modeling (PCA-SM) of Correlated Loads for Uncertainty Analysis of Design Load Envelopes, (June), 1-15.

[56] Tartaruga, I., Cooper, J. E., Sartor, P., Lowenberg, M. H., Coggon, S., \& Lemmens, Y. (2015). Efficient Prediction and Uncertainty Propagation of Correlated Loads. SCITECH, $5-8$.

[57] Castellani, M., Lemmens, Y., \& Cooper, J. E. (2016). Parametric reduced order model approach for rapid dynamic loads prediction, 52, 29-40. 
[58] Bisplinghoff, R. L., Ashley, H., \& Halfman, R. L. (2013). Aeroelasticity. Courier Corporation.

[59] Cornwell, R. E., Craig, R. R., \& Johnson, C. P. (1983). On the application of the modeacceleration method to structural engineering problems. Earthquake engineering \& structural dynamics, 11(5), 679-688.

[60] Sanchez, R. R., Buchschmid, M., \& Gerhard, M. (2016). Model order reduction in structural dynamics, (June), 5-10.

[61] ElSayed, M.S.A., Sedaghati, R., \& Abdo, M. (2009). Accurate stick model development for static analysis of complex aircraft wing-box structures. AIAA journal, 47(9), 20632075.

[62] ElSayed, M.S.A., Contreras, M. A. G., \& Stathopoulos, N. (2013). Monitor Points Method for Loads Recovery in Static/Dynamic Aeroelasticity Analysis with Hybrid Airframe Representation. SAE International Journal of Aerospace, 6(2013-01-2142), 399-407.

[63] Fu, Z. F., \& He, J. (2001). Modal analysis. Butterworth-Heinemann.

[64] MacNeal, R. (1972). The NASTRAN Theoretical Manual (level 15.5): Msr-40. MacNealSchwendler Corporation.

[65] Saad, Y. (1992). Numerical methods for large eigenvalue problems. Manchester University Press.

[66] Albano, E., \& Rodden, W. P. (1969). A doublet-lattice method for calculating lift distributions on oscillating surfaces in subsonic flows. AIAA journal, 7(2), 279-285. 
[67] Harder, R. L., \& Desmarais, R. N. (1972). Interpolation using surface splines. Journal of aircraft, 9(2), 189-191.

[68] Pototzky, A. S., \& Perry III, B. (1986). New and existing techniques for dynamic loads analyses of flexible airplanes. Journal of Aircraft, 23(4), 340-347

[69] Ünay, E., Gürak, D., Özerciyes, V., Uzunoğlu, A., Kestek, H., \& Çıkrıkcı, D. (2012, September). Tool Development for Aircraft Loads Post-Processing. In Proceedings of the 28th International Congress of the Aeronautical Sciences.Uri, K. (2008). Reanalysis of Structures, 151(3). https://doi.org/10.1007/978-1-4020-8198-9

[70] Olsson, A., Sandberg, G., \& Dahlblom, O. (2003). On Latin hypercube sampling for structural reliability analysis. Structural safety, 25(1), 47-68.

[71] McKay, M. D., Beckman, R. J., \& Conover, W. J. (1979). Comparison of three methods for selecting values of input variables in the analysis of output from a computer code. Technometrics, 21(2), 239-245.

[72] Lophaven, S. N., Nielsen, H. B., \& Søndergaard, J. (2002). DACE: a Matlab kriging toolbox (Vol. 2). IMM, Informatics and Mathematical Modelling, The Technical University of Denmark.

[73] Lehmann, E. L. (1951). A general concept of unbiasedness. The Annals of Mathematical Statistics, 22(4), 587-592.

[74] Abt, M. (1994). A note on the product correlation rule. Linear algebra and its applications, 199, 171-177. 
[75] Ababou, R., Bagtzoglou, A. C., \& Wood, E. F. (1994). On the condition number of covariance matrices in kriging, estimation, and simulation of random fields. Mathematical Geology, 26(1), 99-133.

[76] Mardia, K. V., \& Marshall, R. J. (1984). Maximum likelihood estimation of models for residual covariance in spatial regression. Biometrika, 71(1), 135-146.

[77] Zhang, X. (2015). A Tutorial on Restricted Maximum Likelihood Estimation in Linear $\begin{array}{llll}\text { Regression and } & \text { Linear }\end{array}$ http://web.mit.edu/xiuming/www/docs/tutorials/ReML.pdf. [retrieved July 2017]

[78] Franey, M., Ranjan, P., \& Chipman, H. (2011). Branch and bound algorithms for maximizing expected improvement functions. Journal of Statistical Planning and Inference, 141(1), 42-55.

[79] Arief, A., Nappu, M. B., Nizar, A., \& Dong, Z. Y. (2009). Determination of DG allocation with modal participation factor to enhance voltage stability.

[80] Gebreselassie, A., \& Chow, J. H. (1994). Investigation of the effects of load models and generator voltage regulators on voltage stability. International Journal of Electrical Power \& Energy Systems, 16(2), 83-89.

[81] Arief, A., Nappu, M. B., Dong, Z. Y., \& Arief, M. (2010, October). Under voltage load shedding incorporating bus participation factor. In IPEC, 2010 Conference Proceedings(pp. 561-566). IEEE. 
[82] Hashlamoun, W. A., Hassouneh, M. A., \& Abed, E. H. (2009). New results on modal participation factors: Revealing a previously unknown dichotomy. IEEE Transactions on Automatic Control, 54(7), 1439-1449.

[83] MacNeal, R. (1972). The NASTRAN Theoretical Manual (level 15.5): Msr-40. MacNealSchwendler Corporation.

[84] Irvine, T. (2013). Effective modal mass and modal participation factors. Available on the web on site: http://www. vibrationdata. com/tutorials2/ModalMass. pdf.(last access on march 7 2007).

[85] Girard, A., \& Roy, N. A. (1997). Modal effective parameters in structural dynamics. Revue Européenne des éléments finis, 6(2), 233-254.

[86] Kuhar, E. J., \& Stahle, C. V. (1974). Dynamic transformation method for modal synthesis. AIAA Journal, 12(5), 672-678.

[87] Chen, J. T., Chen, K. H., Chen, I. L., \& Liu, L. W. (2003). A new concept of modal participation factor for numerical instability in the dual BEM for exterior acoustics. Mechanics Research Communications, 30(2), 161-174.

[88] Chen, J. T., Hong, H. K., \& Yeh, C. S. (1995). Modal reaction method for modal participation factors in support motion problems. International Journal for Numerical Methods in Biomedical Engineering, 11(6), 479-490.

[89] Wison, E. L., Yuan, M. W., \& Dickens, J. M. (1982). Dynamic analysis by direct superposition of Ritz vector. Earthquake engineering and structural dynamics, 10, 813-821. 
[90] Carlbom, P. F. (2001). Combining MBS with FEM for rail vehicle dynamics analysis. Multibody System Dynamics, 6(3), 291-300.

[91] Salmonte, A. J. (1982). Considerations on the residual contribution in modal analysis. Earthquake Engineering \& Structural Dynamics, 10(2), 295-304.

[92] Photiadis, D. M., Houston, B. H., Liu, X., Bucaro, J. A., \& Marcus, M. H. (2002). Thermoelastic loss observed in a high Q mechanical oscillator. Physica B: Condensed Matter, 316, 408-410.

[93] Wallrapp, O., \& Wiedemann, S. (2002). Simulation of deployment of a flexible solar array. Multibody System Dynamics, 7(1), 101-125.

[94] Chopra, A. K. (1996). Modal analysis of linear dynamic systems: physical interpretation. Journal of structural engineering, 122(5), 517-527.

[95] Chopra, A. K. (2001). Dynamics of structures: Theory and applications. Prentice Hall

[96] Palermo, M., Silvestri, S., Gasparini, G., \& Trombetti, T. (2015). Seismic modal contribution factors. Bulletin of Earthquake Engineering, 13(10), 2867-2891.

[97] Romera, L. E., \& Hernandez, S. (1999). An improved technique for modal contribution factors of dynamic responses. In AIAA/ASME/ASCE/AHS/ASC Structures, Structural Dynamics, and Materials Conference and Exhibit, 40 th, St. Louis, MO (pp. 439-445).

[98] De Silva, C. W. (Ed.). (2005). Vibration and shock handbook. CRC Press.

[99] Wijker, J. J. (2008). Spacecraft structures. Springer Science \& Business Media. 
[100] Kammer, D. C., Cessna, J., \& Kostuch, A. (2005). An Effective Mass measure for selecting free-free target modes. In Proceedings of the 23rd International Modal Analysis Conference, Orlando, Florida.

[101] Li, H., Yang, H., \& Hu, S. L. J. (2006). Modal strain energy decomposition method for damage localization in 3D frame structures. Journal of engineering mechanics, 132(9), 941-951.

[102] Shi, Z., Law, S. S., \& Zhang, L. (2000). Structural damage detection from modal strain energy change. Journal of engineering mechanics, 126(12), 1216-1223.

[103] Shi, Z. Y., Law, S. S., \& Zhang, L. M. (2002). Improved damage quantification from elemental modal strain energy change. Journal of engineering mechanics, 128(5), 521-529.

[104] Bombardier Aerospace (2016). BD100 airframe mass and stiffness distribution. [proprietary]. 